\title{
Decarboxylative Cross-Electrophile Coupling of $N$-Hydroxyphthalimide Esters with Aryl Iodides
}

Kierra M. M. Huihui, ${ }^{\ddagger}$ Jill A. Caputo, ${ }^{\ddagger}$ Zulema Melchor, Astrid M. Olivares, Amanda M. Spiewak, Keywan A. Johnson, Tarah A. DiBenedetto, Seoyoung Kim, Laura K. G. Ackerman, and Daniel J. Weix*

Department of Chemistry, University of Rochester, Rochester, NY, USA 14627

\section{Supporting Information}




\section{Table of Contents}

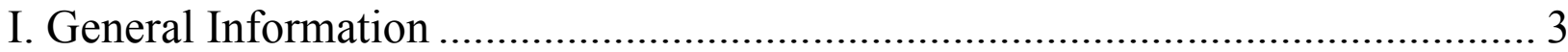

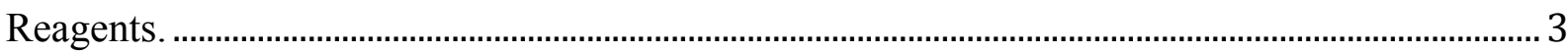

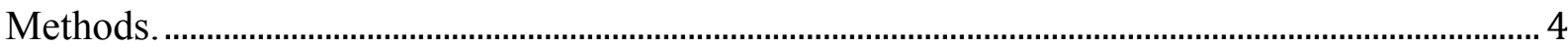

II. Synthesis of $N$-Hydroxyphthalimide esters (NHP esters) ................................ 6

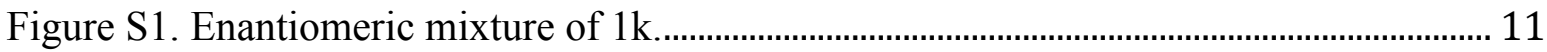

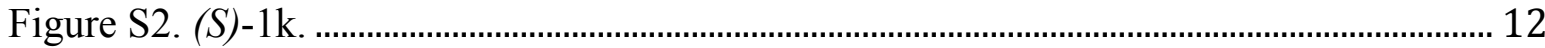

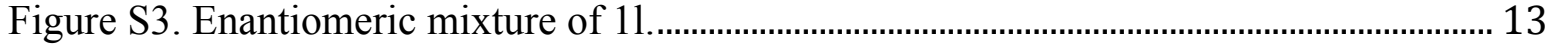

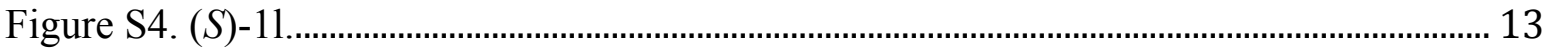

III. Coupling of NHP Esters with Aryl Iodides ................................................ 15

General Procedure for the Coupling of $N$-(acyloxy)Phthalimides and Aryl Iodides................. 15

Large-Scale Procedure conducted on the benchtop in a round-bottom flask.............................. 16

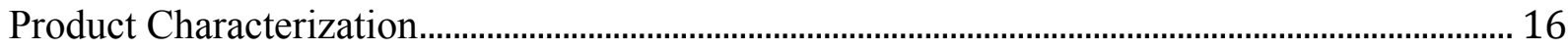

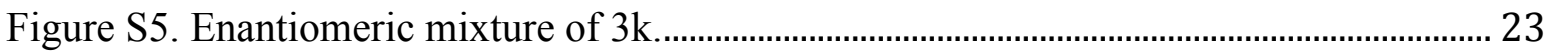

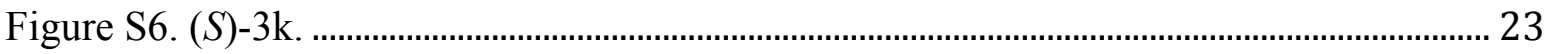

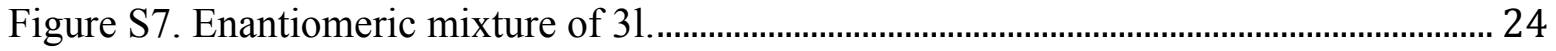

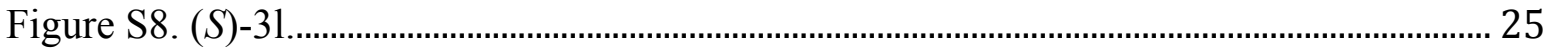

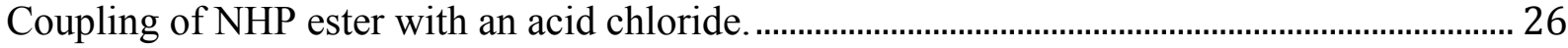

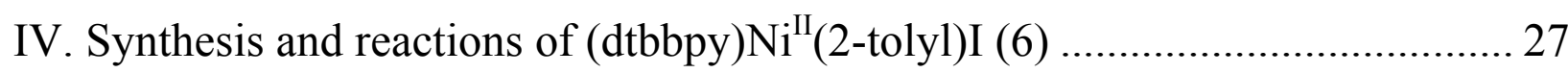

Figure S9. UV-vis of 6 recorded in DMF at rt. ................................................................................... 28

Figure S10. ${ }^{1} \mathrm{H}$ NMR spectra of $6,1 \mathrm{~b}$, and $6+1 \mathrm{~b}$ after stirring overnight in DMF- $d_{7}$ at $\mathrm{rt} . \ldots . . .29$

Figure S11. Paramagnetic ${ }^{1} \mathrm{H}$ NMR spectrum of $6+1 \mathrm{~b}$ after stirring overnight in DMF- $d_{7}$ at rt.

Figure S12. UV-vis spectrum of $6+1 \mathrm{~b}$ after stirring overnight in DMF- $d_{7}$ at rt....................... 31

V. NMR Spectra .............................................................................. 32 


\section{General Information}

\section{Reagents.}

\section{Metals.}

Zinc flake (-325 mesh, Alfa Aesar) and nickel(II) bromide 2-methoxyethyl ether $\left(\mathrm{NiBr}_{2}\right.$ (diglyme), Aldrich) were used as received and stored in a nitrogen filled glovebox. The amount of diglyme present in the $\mathrm{NiBr}_{2}$ (diglyme) was determined by elemental analysis and the amount of $\mathrm{NiBr}_{2}$ (diglyme) was calculated accordingly. (dtbbpy) $\mathrm{NiBr}_{2}$ was synthesized from $\mathrm{NiBr}_{2} \cdot 3 \mathrm{H}_{2} \mathrm{O}$ and dtbbpy in ethanol in analogy to a published procedure. ${ }^{1} \mathrm{Ni}(\mathrm{cod})_{2}$ (Stem Chemicals) was used soon after receiving as this material decomposes over time. Although we had previously experienced success with material from Aldrich, recent (Jan 2016) lots from Aldrich were of poor quality.

\section{Ligands.}

4-4'-di-tert-butyl-2,2'-bipyridine (Aldrich) was used as received. Other ligands tested were from commercial suppliers and used as received.

\section{Solvents.}

Anhydrous $N, N$-dimethylacetamide (DMA), $N, N$-dimethylformamide (DMF), pentane, and THF were obtained by passage through activated alumina and molecular sieves in a solvent purification system and were stored over $4 \AA$ molecular sieves in a nitrogen filled glovebox. Solvents used for organometallic work were further degassed by 3-6 freeze-pump-thaw cycles. DMF- $d_{7}$ and acetone- $d_{6}$ (Cambridge Isotope Laboratories, Inc.) were degassed before use and stored in an argon glovebox.

\section{Other Reagents.}

Dodecane (Aldrich), $\quad N$-(tert-butoxycarbonyl)proline (Alfa Aesar), $N, N^{\prime}-$ dicyclohexylcarbodiimide (Alfa Aesar), N,N'-diisopropylcarbodiimide (Chem-Impex), $N$ hydroxyphthalimide (Alfa Aesar), DMAP (Alfa Aesar), 4-phenylbutyric acid (Aldrich), 3,3dimethylbutyric acid (Alfa Aesar), cyclopentane carboxylic acid (Combi-Blocks), Boc-AspOtBu (Combi-Blocks), Boc-Glu-OtBu (Combi-Blocks), N-Boc-Pro-OH (Alfa Aesar), 4acetylbutyric acid (Combi-Blocks), cholic acid (Sigma Aldrich), and piperidine-3-carboxylic acid (Comb-Blocks) were used as received.

\footnotetext{
${ }^{1}$ Durandetti, M.; Devaud, M.; Périchon, J. New J. Chem. 1996, 20, 659-667.
} 


\section{Substrates.}

1-(5-iodo-1H-indol-1-yl)ethanone ${ }^{2}$ and 4-iodophenylboronic acid pinacol ester ${ }^{3}$ were prepared according to literature procedures. Iodobenzene (Alfa Aesar), 2-iodotoluene (Aldrich), 4iodoacetophenone (Alfa Aesar), ethyl-3-iodobenzoate (Combi-Blocks), and ethyl succinyl chloride (TCI America) were used as received.

\section{Methods.}

\section{NMR Spectroscopy.}

${ }^{1} \mathrm{H}$ and ${ }^{13} \mathrm{C}$ NMR spectra were acquired on 400 and $500 \mathrm{MHz}$ Bruker NMR instruments. NMR chemical shifts are reported in ppm and are referenced to TMS $\left(\delta=0.00 \mathrm{ppm},{ }^{1} \mathrm{H}\right.$ NMR$)$ or the residual solvent peak for $\mathrm{CDCl}_{3}\left(\delta=7.26 \mathrm{ppm},{ }^{1} \mathrm{H} \mathrm{NMR} ; \delta=77.16 \mathrm{ppm},{ }^{13} \mathrm{C} \mathrm{NMR}\right)$. Coupling constants $(J)$ are reported in Hertz. For substrates that existed as rotamers at ambient temperature, the ${ }^{1} \mathrm{H}$ NMR spectrum was obtained at $55^{\circ} \mathrm{C}$.

\section{Gas Chromatography.}

GC analyses were performed on an Agilent 7890A GC equipped with dual DB-5 columns (20 $\mathrm{m} \times 180 \mu \mathrm{m} \times 0.18 \mu \mathrm{m})$, dual FID detectors, and hydrogen as the carrier gas. A sample volume of $1 \mu \mathrm{L}$ was injected at a temperature of $300{ }^{\circ} \mathrm{C}$ and a $100: 1$ split ratio. The initial inlet pressure was 20.3 psi but varied as the column flow was held constant at $1.8 \mathrm{~mL} / \mathrm{min}$ for the duration of the run. The initial oven temperature of $50{ }^{\circ} \mathrm{C}$ was held for $0.46 \mathrm{~min}$ followed by a temperature ramp of $65^{\circ} \mathrm{C} / \mathrm{min}$ up to $300^{\circ} \mathrm{C}$. The temperature was held at $300^{\circ} \mathrm{C}$ for $3 \mathrm{~min}$. The total run time was $\sim 7.3$ min and the FID temperature was $325^{\circ} \mathrm{C}$.

\section{GC/MS Analysis}

GC/MS analyses were performed on a Shimadzu GCMS-QP2010 equipped with an RTX-XLB column $(30 \mathrm{~m} \times 0.25 \mathrm{~mm} \times 0.28 \mu \mathrm{m})$ with a quadrupole mass analyzer using helium as the carrier gas. The analysis method used in all cases was $5 \mu \mathrm{L}$ injection of sample, an injection temp of $225^{\circ} \mathrm{C}$, and a $25: 1$ split ratio. The initial inlet pressure was 7.8 psi, but varied as the column flow was held constant at $1.0 \mathrm{~mL} / \mathrm{min}$ for the duration of the run. The interface temperature was held at $250{ }^{\circ} \mathrm{C}$, and the ion source $(\mathrm{EI}+, 30 \mathrm{eV})$ was held at $250{ }^{\circ} \mathrm{C}$. The initial

\footnotetext{
${ }^{2}$ Lai, A.; Kahraman, M.; Govek, S.; Nagasawa, J.; Bonnefous, C.; Julien, J.; Douglas, K.; Sensintaffar, J.; Lu, N.; Lee, K.-J.; Aparicio, A.; Kaufman, J.; Qian, J.; Shao, G.; Prudente, R.; Moon, M. J.; Joseph, J. D.; Darimont, B.; Brigham, D.; Grillot, K.; Heyman, R.; Rix, P. J.; Hager, J. H.; Smith, N. D. J. Med. Chem. 2015, 58, $4888-4904$.

${ }^{3}$ Matteson, D. S.; Man, H.-W. J. Org. Chem. 1994, 59, 5734-5741.
} 
oven temperature was held at $50^{\circ} \mathrm{C}$ for $2 \mathrm{~min}$ with the detector off, followed by a temperature ramp, with the detector on, to $280{ }^{\circ} \mathrm{C}$ at $40{ }^{\circ} \mathrm{C} / \mathrm{min}$. The temperature was held at $280{ }^{\circ} \mathrm{C}$ for 3 min. Total run time was $11.75 \mathrm{~min}$.

\section{Supercritical Fluid Chromatography (SFC) Analysis}

SFC analysis was performed on a Jasco Analytical and Semi-Preparative SFC instrument equipped with a column oven $\left(35^{\circ} \mathrm{C}\right)$, photodiode array detector, a back pressure regulator $(12.0$ $\mathrm{MPa}$ ), and a carbon dioxide pump. Chiralpak IA and IC columns were used for separation of enantiomers. All analyses were performed on isolated samples. For each analysis, $\sim 1-2 \mathrm{mg}$ of material was used and diluted with $1 \mathrm{~mL}$ of diethyl ether. Racemic samples were used for method determination and were prepared by combining equal amounts of enantiopure materials.

\section{Low Resolution Mass Spectrometry (LRMS) Analysis}

LRMS analyses were performed on a Thermo LTQ Velos LC/MS equipped with an electrospray (ESI) probe operating in positive ion mode (ESI+) with an ion trap mass analyzer. Direct injection analysis was employed in all cases with a sample solution in methanol.

\section{Elemental Analysis.}

Elemental analyses were performed by CENTC Elemental Analysis Facility at University of Rochester, funded by NSF CHE-0650456.

\section{Infrared Spectroscopy.}

Infrared (IR) spectra were recorded on a Shimadzu IRAffinity-1 Fourier Transform Infrared Spectrophotometer and are reported in wavenumbers $\left(\mathrm{cm}^{-1}\right)$.

\section{UV-Vis spectroscopy.}

UV-Vis spectra (300-900 nm) were recorded on a HP 8452A spectrometer using quartz, screw-cap cuvettes to exclude oxygen. The samples were prepared in a glove box under Ar.

\section{Chromatography.}

Chromatography was performed on silica gel (EMD, silica gel 60, particle size 0.040$0.063 \mathrm{~mm}$ ) using standard flash techniques or on a Teledyne Isco Rf-200 (detection at $210 \mathrm{~nm}$ ). Products were visualized by UV, iodine stain, ceric ammonium molybdate stain, or $\mathrm{KMnO}_{4}$ stain. 


\section{Synthesis of $\mathrm{N}$-Hydroxyphthalimide esters (NHP esters)}

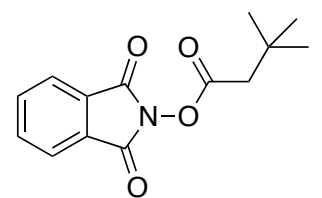

\section{1,3-dioxoisoindolin-2-yl 3,3-dimethylbutanoate (1a).}

A round bottom flask containing a teflon-coated magnetic stir bar was charged with tertbutylacetic acid $(5.50 \mathrm{~mL}, 43.0 \mathrm{mmol}, 1.00$ equiv) and dry EtOAc (420 mL). After sequential addition of $N, N^{\prime}$-dicyclohexylcarbodiimide (10.5 g, $64.5 \mathrm{mmol}, 1.50$ equiv), DMAP (263 mg, $2.15 \mathrm{mmol}, 0.0500$ equiv), and $N$-hydroxyphthalimide $(4.33 \mathrm{~g}, 26.6 \mathrm{mmol}, 1.66$ equiv), the reaction mixture was allowed to stir overnight at room temperature. After this time, the heterogeneous mixture was filtered and the filtrate was concentrated under reduced pressure. Recrystallization from acetone yielded product (10.0 g, $38.4 \mathrm{mmol}, 89 \%)$ as a colorless solid. ${ }^{1} \mathrm{H}$ NMR (400 MHz; $\left.\mathrm{CDCl}_{3}\right): \delta 7.89(\mathrm{dd}, J=5.4,3.0 \mathrm{~Hz}, 2 \mathrm{H}), 7.79(\mathrm{dd}, J=5.5,3.0 \mathrm{~Hz}, 2 \mathrm{H})$, $2.53(\mathrm{~s}, 2 \mathrm{H}), 1.17(\mathrm{~s}, 9 \mathrm{H})$.

${ }^{13} \mathrm{C} \mathrm{NMR}\left(126 \mathrm{MHz}, \mathrm{CDCl}_{3}\right): \delta$ 168.0, 162.2, 134.8, 129.1, 124.1, 44.7, 31.4, 29.6. $\operatorname{mp} 92-93{ }^{\circ} \mathrm{C}$ IR ( $\left.\mathrm{cm}^{-1}\right): 2936,2361,1809,1782,1736,1624,1470,1366,1346,1319,1180,1088,1034,968$, 876, 810, 787, 698.

LRMS (ESI+) $m / z: 262.2\left[\mathrm{M}+\mathrm{H}^{+}\right], 284.2\left[\mathrm{M}+\mathrm{Na}^{+}\right]$.

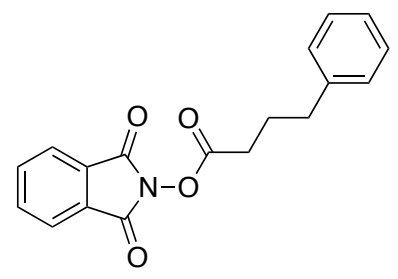

\section{1,3-dioxoisoindolin-2-yl 4-phenylbutanoate (1b).}

The procedure was adapted from the method of Overman and coworkers. ${ }^{4}$ A round bottom flask containing a teflon-coated magnetic stir bar was charged with 4-phenylbutyric acid (2.63 g, 16.0 mmol, 1.00 equiv) and dry THF $(75 \mathrm{~mL})$ under argon. After sequential addition of $N$ hydroxyphthalimide (4.34 g, $26.6 \mathrm{mmol}, 1.66$ equiv), DMAP (98.0 mg, $0.800 \mathrm{mmol}, 0.0500$ equiv), and $N, N^{\prime}$-diisopropylcarbodiimide (3.03 g, $2.40 \mathrm{mmol}, 1.50$ equiv), the reaction mixture was allowed to stir overnight at room temperature. After this time, the heterogeneous mixture was filtered and the filtrate was concentrated under reduced pressure. The crude product was

\footnotetext{
${ }^{4}$ Pratsch, G.; Lackner, G. L.; Overman, L. E. J. Org. Chem. 2015, 80, 6025-6036.
} 
purified by silica gel chromatography $\left(80: 20\right.$ hexanes/EtOAc, $\left.R_{\mathrm{f}}=0.31\right)$ to yield product $(3.86 \mathrm{~g}$, $12.5 \mathrm{mmol}, 78 \%)$ as a colorless solid.

${ }^{1} \mathrm{H}$ NMR (400 MHz; $\left.\mathrm{CDCl}_{3}\right): \delta 7.90(\mathrm{dd}, J=5.2,3.1 \mathrm{~Hz}, 2 \mathrm{H}), 7.79(\mathrm{dd}, J=5.4,3.0 \mathrm{~Hz}, 2 \mathrm{H})$, $7.31(\mathrm{t}, J=7.4 \mathrm{~Hz}, 2 \mathrm{H}), 7.24(\mathrm{t}, J=7.9 \mathrm{~Hz}, 3 \mathrm{H}), 2.78(\mathrm{t}, J=7.5 \mathrm{~Hz}, 2 \mathrm{H}), 2.67(\mathrm{t}, J=7.3 \mathrm{~Hz}$, 2H), 2.17-2.08 (m, 2H).

${ }^{1} \mathrm{H}$ NMR (500 MHz; DMF- $\left.d_{7}\right): \delta 7.32(\mathrm{dd}, J=11.6,5.8 \mathrm{~Hz}, 5 \mathrm{H}), 7.24(\mathrm{dd}, J=6.7,5.0 \mathrm{~Hz}, 1 \mathrm{H})$, $2.80(\mathrm{dd}, J=6.2,4.6 \mathrm{~Hz}, 6 \mathrm{H}), 2.06(\mathrm{dd}, J=13.2,6.9 \mathrm{~Hz}, 3 \mathrm{H})$.

${ }^{13} \mathrm{C} \mathrm{NMR}\left(126 \mathrm{MHz}, \mathrm{CDCl}_{3}\right): \delta 169.6,162.1,140.8,134.9,129.1,128.7,128.7,126.4,124.1$, $34.8,30.4,26.5$.

$\mathrm{mp} 83-86^{\circ} \mathrm{C}$

IR (cm $\left.{ }^{-1}\right): 2928,2855,1805,1778,1732,1612,1458,1366,1219,1184,1146,1123,1088,1072$, 1030, 918, 760, 706, 664.

LRMS (ESI+) $m / z: 310.2\left[\mathrm{M}+\mathrm{H}^{+}\right], 332.2\left[\mathrm{M}+\mathrm{Na}^{+}\right]$.

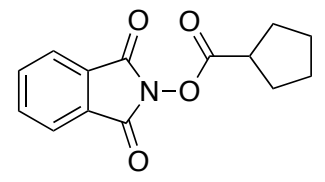

\section{1,3-dioxoisoindolin-2-yl cyclopentanecarboxylate (1c).}

The procedure was adapted from the method of Overman and coworkers. ${ }^{4}$ A round bottom flask containing a teflon-coated magnetic stir bar was charged with cyclopentane carboxylic acid (4.33 $\mathrm{mL}, 40.0 \mathrm{mmol}, 1.00$ equiv) and dry THF (150 mL) under nitrogen. After sequential addition of $N$-hydroxyphthalimide (10.8 g, $66.4 \mathrm{mmol}, 1.66$ equiv), DMAP (977 mg, $8.00 \mathrm{mmol}, 0.200$ equiv), and $N, N^{\prime}$-diisopropylcarbodiimide $(9.30 \mathrm{~mL}, 60.0 \mathrm{mmol}, 1.50$ equiv), the reaction mixture was allowed to stir overnight at room temperature. After this time, the heterogeneous mixture was filtered and the filtrate was concentrated under reduced pressure. The crude product was purified by silica gel chromatography $\left(90: 10\right.$ hexanes/EtOAc, $\left.R_{f}=0.13\right)$ to yield product (2.89 g, $11.2 \mathrm{mmol}, 28 \%)$ as a colorless solid.

${ }^{1} \mathrm{H}$ NMR (400 MHz; $\mathrm{CDCl}_{3}$ ): $\delta 7.89(\mathrm{dd}, J=5.4,3.1 \mathrm{~Hz}, 2 \mathrm{H}), 7.79(\mathrm{dt}, J=5.9,3.2 \mathrm{~Hz}, 2 \mathrm{H})$, 3.16-3.08 (m, 1H), 2.11-1.99 (m, 4H), 1.81-1.75 (m, 2H), 1.72-1.65 (m, 2H).

${ }^{13} \mathrm{C} \mathrm{NMR}\left(126 \mathrm{MHz}, \mathrm{CDCl}_{3}\right): \delta 173.0,162.3,134.8,129.2,124.0,40.8,30.4,26.1$.

$\operatorname{mp} 62-65^{\circ} \mathrm{C}$

IR $\left(\mathrm{cm}^{-1}\right): 2967,2874,1813,1782,1740,1608,1589,1524,1466,1450,1362,1288,1258,1184$, 1080, 972, 876, 694 .

LRMS (ESI+) $m / z: 260.1\left[\mathrm{M}+\mathrm{H}^{+}\right], 282.1\left[\mathrm{M}+\mathrm{Na}^{+}\right]$. 


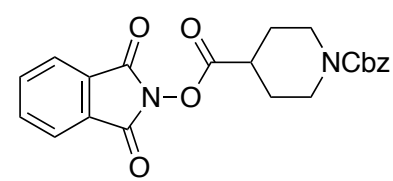

1-benzyl 4-(1,3-dioxoisoindolin-2-yl) piperidine-1,4-dicarboxylate (1d).

1-((benzyloxy)carbonyl)piperidine-4-carboxylic acid was prepared from piperidine-4-carboxylic acid (3.23 g, $25.0 \mathrm{mmol}$ ) based on the literature procedure and was used without purification. ${ }^{5}$ The NHP ester was prepared by an adapted procedure from Overman and co-workers. ${ }^{4}$

To an oven dried round bottom flask equipped with a stirbar was added a solution of 1((benzyloxy)carbonyl)piperidine-4-carboxylic acid (crude, approximately $25.0 \mathrm{mmol}$ ) in dry THF (248 mL) under nitrogen. $N$-hydroxyphthalimide (6.12 g, $37.5 \mathrm{mmol}, 1.5$ equiv), DMAP (0.611 g, $5.00 \mathrm{mmol}, 0.200$ equiv), and $N, N^{\prime}$-diisopropylcarbodiimide (4.65 mL, $30.0 \mathrm{mmol}, 1.20$ equiv) were sequentially added, and the reaction mixture was stirred overnight. Upon completion, the reaction mixture was filtered and concentrated under reduced pressure. The resulting solid that formed was then transferred to a filter funnel and washed using ethyl acetate $(200 \mathrm{~mL})$, and the filtrate was concentrated under reduced pressure and purified by column chromatography (70:30, hexanes/EtOAc, $\left.\mathrm{R}_{\mathrm{f}}=0.21\right)$ to afford a viscous liquid. Hot methylcyclohexane was added, and the resulting solution was concentrated under reduced pressure. This process was repeated several times until a white solid was obtained, which was dried under high vacuum (2.90 g, $7.10 \mathrm{mmol}, 28 \%$ yield $)$.

${ }^{1} \mathrm{H}$ NMR (500 MHz; $\left.\mathrm{CDCl}_{3} ; 55^{\circ} \mathrm{C}\right): \delta$ 7.90-7.79 (m, 4H), 7.37-7.31 (m, 5H), $5.14(\mathrm{~s}, 2 \mathrm{H}), 4.10$ $(\mathrm{s}, 2 \mathrm{H}), 3.12(\mathrm{t}, J=10.2 \mathrm{~Hz}, 2 \mathrm{H}), 2.94(\mathrm{tt}, J=9.6,4.5 \mathrm{~Hz}, 1 \mathrm{H}), 2.09(\mathrm{~d}, J=10.5 \mathrm{~Hz}, 2 \mathrm{H}), 1.90(\mathrm{t}$, $J=10.0 \mathrm{~Hz}, 2 \mathrm{H})$.

${ }^{13} \mathrm{C} \mathrm{NMR}\left(126 \mathrm{MHz}, \mathrm{CDCl}_{3}\right): \delta 170.6,162.0,155.2,136.8,134.9,129.0,128.7,128.2,128.0$, 124.1, 67.4, 42.9, 38.5, 27.8 .

mp $96-98{ }^{\circ} \mathrm{C}$

IR ( $\left.\mathrm{cm}^{-1}\right): 2951,2338,1813,1786,1744,1686,1470,1450,1362,1227,1130,1065,1018,995$, $880,756,698$.

LRMS (ESI+) $m / z: 431.2\left[\mathrm{M}+\mathrm{Na}^{+}\right], 447.1\left[\mathrm{M}+\mathrm{K}^{+}\right]$.

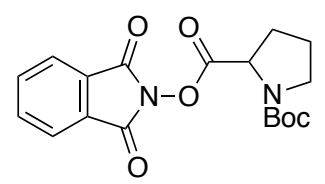

1-(tert-butyl) 2-(1,3-dioxoisoindolin-2-yl) pyrrolidine-1,2-dicarboxylate (1e).

\footnotetext{
${ }^{5}$ Reich, M.; Schunk, S.; Jostock, R.; Hees, S.; Germann, T.; Engels, M. F.-M. Substituted Disulfonamide Compounds US2010152158 (A1) - 2010-06-17.
} 
A round bottom flask equipped with a teflon-coated stirbar was charged with $N$ hydroxyphthalimide (1.52 g, $9.29 \mathrm{mmol}, 1.00$ equiv), $N, N^{\prime}$-dicyclohexylcarbodiimide (1.92 g, $9.29 \mathrm{mmol}, 1.00$ equiv), and dry EtOAc $(25 \mathrm{~mL})$. After the reaction mixture was stirred, $\mathrm{N}$-(tertbutoxycarbonyl)proline (2.00 g, $9.29 \mathrm{mmol}, 1.00$ equiv) and additional EtOAc $(25 \mathrm{~mL})$ was added and the reaction mixture was allowed to stir overnight at room temperature. Stirring was stopped and the reaction mixture was allowed to stand for one day, then filtered through a short silica plug and concentrated under reduced pressure to afford a white solid (2.26 g, $6.27 \mathrm{mmol}$, $67 \%$ yield).

Due to the existence of rotamers at ambient temperature, the ${ }^{1} \mathrm{H}$ NMR spectrum was obtained at $55{ }^{\circ} \mathrm{C}$ :

${ }^{1} \mathrm{H}$ NMR $\left(500 \mathrm{MHz} ; \mathrm{CDCl}_{3} ; 55^{\circ} \mathrm{C}\right): \delta 7.86(\mathrm{dd}, J=5.0,2.6 \mathrm{~Hz}, 2 \mathrm{H}), 7.76(\mathrm{dd}, J=4.7,2.3 \mathrm{~Hz}$, 2H), $4.61(\mathrm{~s}, 1 \mathrm{H}), 3.60(\mathrm{~s}, 1 \mathrm{H}), 3.50(\mathrm{~s}, 1 \mathrm{H}), 2.38(\mathrm{dd}, J=13.5,7.3 \mathrm{~Hz}, 2 \mathrm{H}), 2.13-2.04(\mathrm{~m}, 1 \mathrm{H})$, $1.96(\mathrm{dt}, J=11.7,5.6 \mathrm{~Hz}, 1 \mathrm{H}), 1.51(\mathrm{~s}, 9 \mathrm{H})$.

${ }^{13} \mathrm{C} \mathrm{NMR}\left(126 \mathrm{MHz}, \mathrm{CDCl}_{3}\right): \delta 169.7,161.8,153.6,134.9,128.9,124.0,81.2,57.3,46.3,31.5$, 28.2, 23.6.

mp $155-157^{\circ} \mathrm{C}$ (lit. $\left.{ }^{6} \mathrm{mp} 68-69^{\circ} \mathrm{C}\right)$

IR ( $\left.\mathrm{cm}^{-1}\right)$ : 2936, 2361, 1786, 1736, 1690, 1431, 1389, 1366, 1273, 1223, 1123, 1072, 1015, 880, $749,698$.

LRMS (ESI+) $m / z: 383.2\left[\mathrm{M}+\mathrm{Na}^{+}\right], 399.1\left[\mathrm{M}+\mathrm{K}^{+}\right]$.

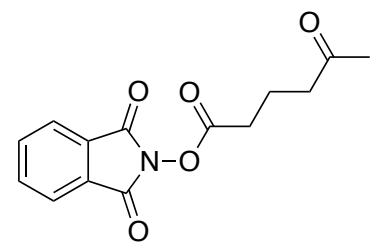

\section{1,3-dioxoisoindolin-2-yl 5-oxohexanoate (1i).}

The procedure was adapted from the method of Overman and coworkers. ${ }^{4}$ A round bottom flask containing a teflon-coated magnetic stir bar was charged with 4-acetylbutyric acid (650 mg, 5.00 mmol, 1.00 equiv) and dry THF $(25 \mathrm{~mL})$ under argon. After sequential addition of $N$ hydroxyphthalimide (1.35 g, $8.30 \mathrm{mmol}, 1.66$ equiv), DMAP (30.0 mg, $0.250 \mathrm{mmol}, 0.0500$ equiv) and $N, N^{\prime}$-diisopropylcarbodiimide (946 $\mathrm{mg}, 7.50 \mathrm{mmol}, 1.50$ equiv), the reaction was allowed to stir overnight at room temperature. After this time, the heterogeneous mixture was filtered and the filtrate was concentrated under reduced pressure. The crude product was purified by silica gel chromatography $\left(80: 20\right.$ hexanes/EtOAc, $\left.\mathrm{R}_{\mathrm{f}}=0.12\right)$ to yield product as a colorless solid (812 mg, $2.95 \mathrm{mmol}, 59 \%$ yield).

\footnotetext{
${ }^{6}$ Il'ina, A. V.; Davidovich, Y. A.; Rogozhin, S. V. Bull. Acad. Sci. USSR, Div. Chem. Sci. (Engl. Transl.) 1984, 33,
} 69-70. 
${ }^{1} \mathrm{H}$ NMR (400 MHz; $\left.\mathrm{CDCl}_{3}\right): \delta 7.89(\mathrm{dd}, J=5.5,3.1 \mathrm{~Hz}, 2 \mathrm{H}), 7.79(\mathrm{dd}, J=5.4,3.1 \mathrm{~Hz}, 2 \mathrm{H})$, $2.73(\mathrm{t}, J=7.0 \mathrm{~Hz}, 2 \mathrm{H}), 2.65(\mathrm{t}, J=7.1 \mathrm{~Hz}, 2 \mathrm{H}), 2.18(\mathrm{~s}, 3 \mathrm{H}), 2.05$ (quintet, $J=7.0 \mathrm{~Hz}, 2 \mathrm{H}$ ).

${ }^{13} \mathrm{C}$ NMR (126 MHz, $\left.\mathrm{CDCl}_{3}\right): \delta$ 207.7, 169.4, 162.1, 134.9 129.0, 124.1, 41.6, 30.2, 30.1, 18.7. $\operatorname{mp} 90-91{ }^{\circ} \mathrm{C}$

IR (cm $\left.{ }^{-1}\right): 2951,1813,1786,1736,1709,1609,1362,1184,1134,1096,1053,972,880$, 795, 698.

LRMS (ESI+) $m / z: 276.2\left[\mathrm{M}+\mathrm{H}^{+}\right], 298.1\left[\mathrm{M}+\mathrm{Na}^{+}\right]$.

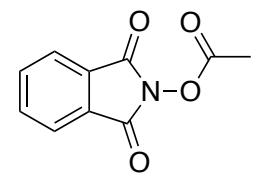

\section{1,3-dioxoisoindolin-2-yl acetate $(1 \mathrm{j})$.}

The procedure was modified from the method of Seligman and coworkers. ${ }^{7}$ Instead of DCC, DIC was used. A round bottom flask containing a telfon-coated magnetic stir bar was charged with $N$ hydroxyphthalimide (2.80 g, $17.4 \mathrm{mmol}, 1.00$ equiv), and $N, N^{\prime}$-diisopropylcarbodiimide (1.79 $\mathrm{mL}, 17.4 \mathrm{mmol}, 1.00$ equiv). Dry EtOAc $(250 \mathrm{~mL})$ was then added followed by acetic acid (1.00 $\mathrm{mL}, 17.4 \mathrm{mmol}, 1.00$ equiv) and the reaction was stirred open to air at room temperature overnight. The reaction mixture became cloudy and a white solid precipitated from the solution, which was removed via vacuum filtration. The filtrate was dried with $\mathrm{Na}_{2} \mathrm{SO}_{4}$ and concentrated under reduced pressure to give the crude product. Recrystallization of the residue from hot ethanol provided product as a colorless solid (3.10 g, $15.1 \mathrm{mmol}, 87 \%$ yield). Analytical data matched those reported in the literature.

${ }^{1} \mathrm{H} \mathrm{NMR}\left(500 \mathrm{MHz} ; \mathrm{CDCl}_{3}\right): \delta 7.89(\mathrm{~s}, 2 \mathrm{H}), 7.80(\mathrm{~s}, 2 \mathrm{H}), 2.40(\mathrm{~d}, J=0.5 \mathrm{~Hz}, 3 \mathrm{H})$.

${ }^{13} \mathrm{C} \mathrm{NMR}\left(126 \mathrm{MHz}, \mathrm{CDCl}_{3}\right): \delta 166.7,162.0,134.9,129.0,124.1,17.8$.

$\mathrm{mp} 160-165^{\circ} \mathrm{C}\left(\mathrm{lit}^{7} \mathrm{mp} 185^{\circ} \mathrm{C}\right)$

IR (cm $\left.{ }^{-1}\right): 1809,1786,1736,1373,1157,1080,1007,968,880,837,694$.

LRMS (ESI+) $m / z: 206.1\left[\mathrm{M}+\mathrm{H}^{+}\right], 228.1\left[\mathrm{M}+\mathrm{Na}^{+}\right]$.

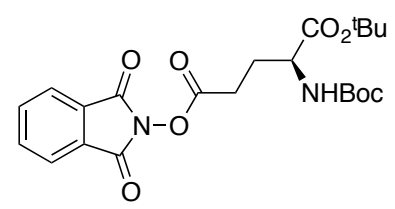

\section{(S)-1-(tert-butyl) 5-(1,3-dioxoisoindolin-2-yl) (tert-butoxycarbonyl)-glutamate (1k).}

The procedure was adapted from the method of Overman and coworkers. ${ }^{4}$ A round bottom flask containing a teflon-coated magnetic stir bar was charged with Boc-Glu-OtBu (1.52 g, 5.00

\footnotetext{
${ }^{7}$ Saljoughian, M.; Morimoto, H.; Williams, P. G.; Than, C.; Seligman, S. J. J. Org. Chem. 1996, 61, 9625-9628.
} 
mmol, 1.00 equiv) and dry THF $(25 \mathrm{~mL})$ under argon. After sequential addition of $\mathrm{N}$ hydroxyphthalimide (1.35 g, $8.3 \mathrm{mmol}, 1.66$ equiv), DMAP (30.0 mg, $0.250 \mathrm{mmol}, 0.0500$ equiv), and $N, N^{\prime}$-diisopropylcarbodiimide ( $946 \mathrm{mg}, 7.50 \mathrm{mmol}, 1.50$ equiv), the reaction was allowed to stir overnight at room temperature. After this time, the heterogeneous mixture was filtered and the filtrate was concentrated under reduced pressure. The crude product was purified by silica gel chromatography $\left(80: 20\right.$ hexanes/EtOAc, $\left.\mathrm{R}_{\mathrm{f}}=0.23\right)$ to yield product as a colorless solid (1.74 g, $3.88 \mathrm{mmol}, 72 \%$ yield).

$[\alpha]_{\mathrm{D}}^{23}+13.3\left(\mathrm{c}=1.08, \mathrm{CHCl}_{3}\right)$

${ }^{1} \mathrm{H}$ NMR (400 MHz; $\left.\mathrm{CDCl}_{3}\right): \delta 7.89(\mathrm{dd}, J=5.5,3.1 \mathrm{~Hz}, 2 \mathrm{H}), 7.79(\mathrm{dd}, J=5.4,3.1 \mathrm{~Hz}, 2 \mathrm{H})$, $5.19(\mathrm{~d}, J=6.7 \mathrm{~Hz}, 1 \mathrm{H}), 4.28$ (d, $J=5.0 \mathrm{~Hz}, 1 \mathrm{H}), 2.85-2.68$ (m, 2H), 2.35-2.29 (m, 1H), 2.11$2.02(\mathrm{~m}, 1 \mathrm{H}), 1.47(\mathrm{~d}, J=14.3 \mathrm{~Hz}, 18 \mathrm{H})$.

${ }^{13} \mathrm{C}$ NMR $\left(126 \mathrm{MHz}, \mathrm{CDCl}_{3}\right): \delta 170.9,169.1,161.9,155.5,134.9,129.0,124.1,82.7,80.1,53.2$, 28.4, 28.1, 27.5.

$\mathrm{mp} 84-85^{\circ} \mathrm{C}$

IR $\left(\mathrm{cm}^{-1}\right): 3368,2978,2936,2361,2338,1813,1786,1740,1690,1508,1366,1150,1072,1018$, 976, 876, 787, 698.

LRMS (ESI+) $m / z: 471.2\left[\mathrm{M}+\mathrm{Na}^{+}\right]$.

SFC analysis (Daicel Chiralpak IA ( $0.46 \mathrm{~cm} \mathrm{ID} \times 25 \mathrm{~cm} \mathrm{~L}), 25 \% i-\mathrm{PrOH}$ in $\mathrm{CO}_{2}, 3 \mathrm{~mL} / \mathrm{min}, \lambda=$ $\left.220 \mathrm{~nm}, 35^{\circ} \mathrm{C}, 12.0 \mathrm{MPa}\right): \mathrm{tR}[\mathrm{min}]=1.9(1.55 \%), 2.5$ (98.45\%), 97\% ee.

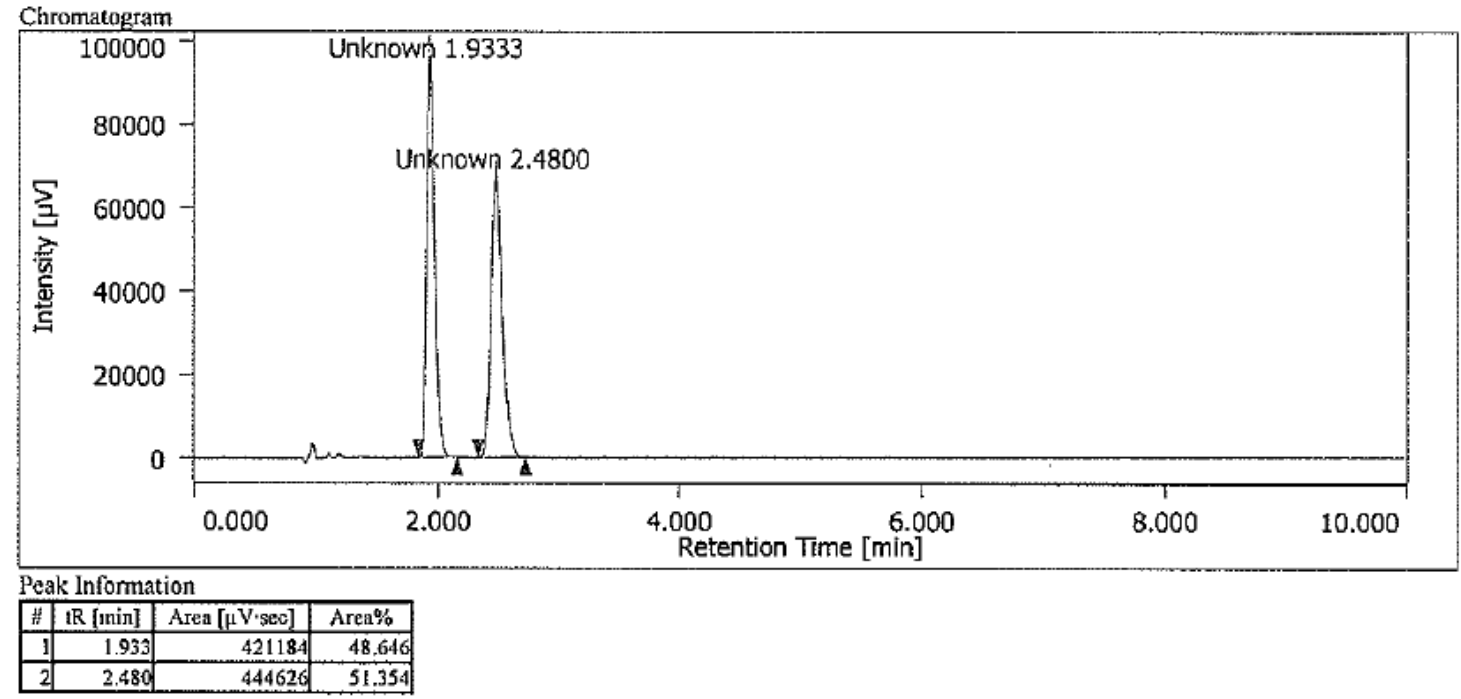

Figure S1. Enantiomeric mixture of $1 \mathbf{k}$. 


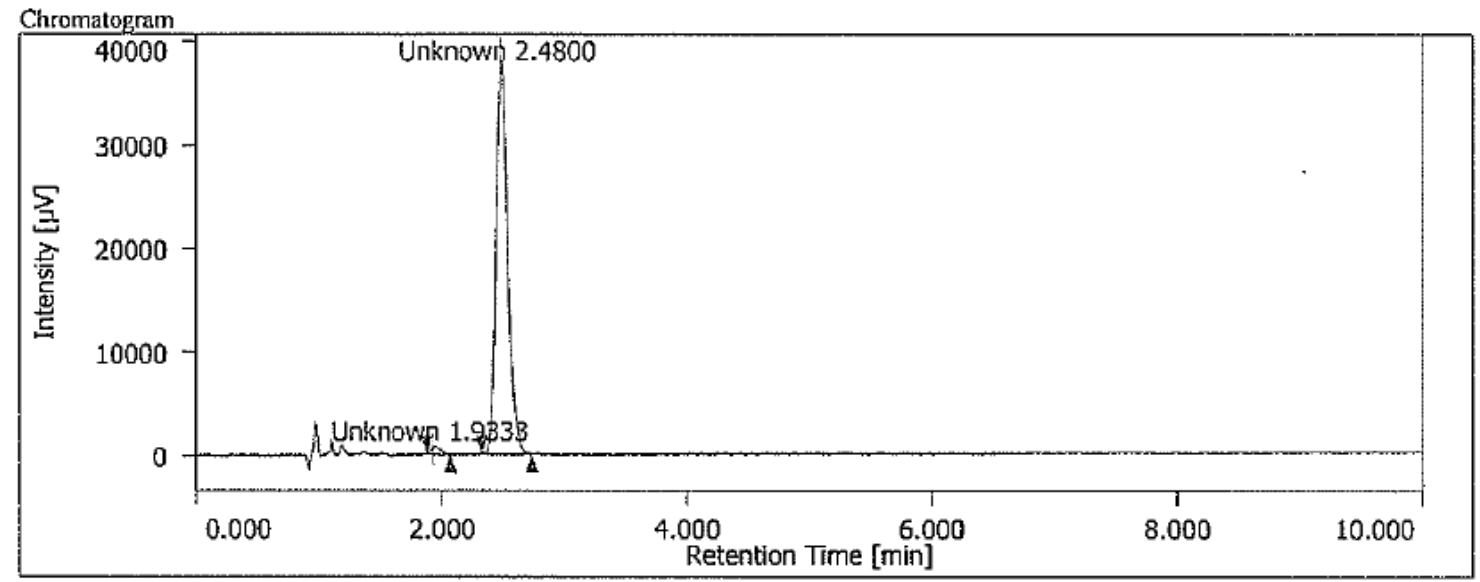

Peak Information

\begin{tabular}{|l|l|l|l|}
\hline$i t$ & RR [min] & Area [1 V.sec] & Area\% \\
\hline & &
\end{tabular}

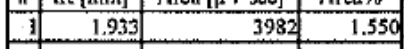

Figure S2. (S)-1k.

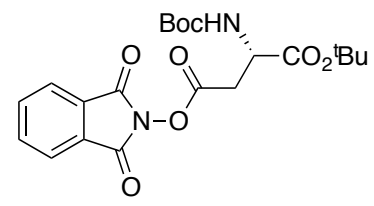

\section{(S)-1-(tert-butyl) 3-(1,3-dioxoisoindolin-2-yl)-2-((tert-butoxycarbonyl)amino)malonate (11).}

The procedure was adapted from the method of Overman and coworkers. ${ }^{4}$ A round bottom flask containing a teflon-coated magnetic stir bar was charged with Boc-Asp-OtBu (4.63 g, 16.0 mmol, 1.00 equiv) and dry THF $(75 \mathrm{~mL})$ under argon. After sequential addition of $N$ hydroxyphthalimide (4.34 g, $26.6 \mathrm{mmol}, 1.66$ equiv), DMAP (98.0 mg, $0.800 \mathrm{mmol}, 0.0500$ equiv), and $N, N^{\prime}$-diisopropylcarbodiimide $(3.03 \mathrm{~g}, 24.0 \mathrm{mmol}, 1.50$ equiv), the reaction was allowed to stir overnight at room temperature. After this time, the heterogeneous mixture was filtered and the filtrate was concentrated under reduced pressure. The crude product was purified by silica gel chromatography $\left(80: 20\right.$ hexanes/EtOAc, $\left.R_{f}=0.23\right)$ to yield product $(5.60 \mathrm{~g}, 12.9$ mmol, $81 \%$ yield) as a white solid.

$[\alpha]_{\mathrm{D}}^{23}+32.6\left(\mathrm{c}=1.01, \mathrm{CHCl}_{3}\right)$

${ }^{1} \mathrm{H}$ NMR $\left(400 \mathrm{MHz} ; \mathrm{CDCl}_{3}\right): \delta 7.89(\mathrm{dd}, J=5.1,3.1 \mathrm{~Hz}, 2 \mathrm{H}), 7.79(\mathrm{dd}, J=5.2,3.1 \mathrm{~Hz}, 2 \mathrm{H})$, 5.51-5.49 (m, 1H), 4.59-4.57 (m, 1H), $3.28(\mathrm{dd}, J=24.4,4.1 \mathrm{~Hz}, 2 \mathrm{H}), 1.47(\mathrm{~d}, J=7.6 \mathrm{~Hz}, 18 \mathrm{H})$. ${ }^{13} \mathrm{C}$ NMR $\left(126 \mathrm{MHz}, \mathrm{CDCl}_{3}\right): \delta 168.8,167.6,161.7,155.4,134.9,128.9,124.1,83.4,80.3,50.3$, 34.3, 28.4, 27.9.

mp $124-125^{\circ} \mathrm{C}$

IR $\left(\mathrm{cm}^{-1}\right): 3372,2970,2361,1809,1786,1748,1694,1501,1362,1246,1215,1153,1103,1065$, $968,787,694$. 
LRMS (ESI+) $m / z: 457.2\left[\mathrm{M}+\mathrm{Na}^{+}\right]$.

SFC analysis (Daicel Chiralpak IA $(0.46 \mathrm{~cm} \mathrm{ID} \times 25 \mathrm{~cm} \mathrm{~L}), 10 \% i-\mathrm{PrOH}$ in $\mathrm{CO}_{2}, 4 \mathrm{~mL} / \mathrm{min}, \lambda=$ $\left.220 \mathrm{~nm}, 35^{\circ} \mathrm{C}, 12.0 \mathrm{MPa}\right): \mathrm{tR}[\mathrm{min}]=3.2(0.2 \%), 3.9(99.8 \%),>98 \%$ ee.

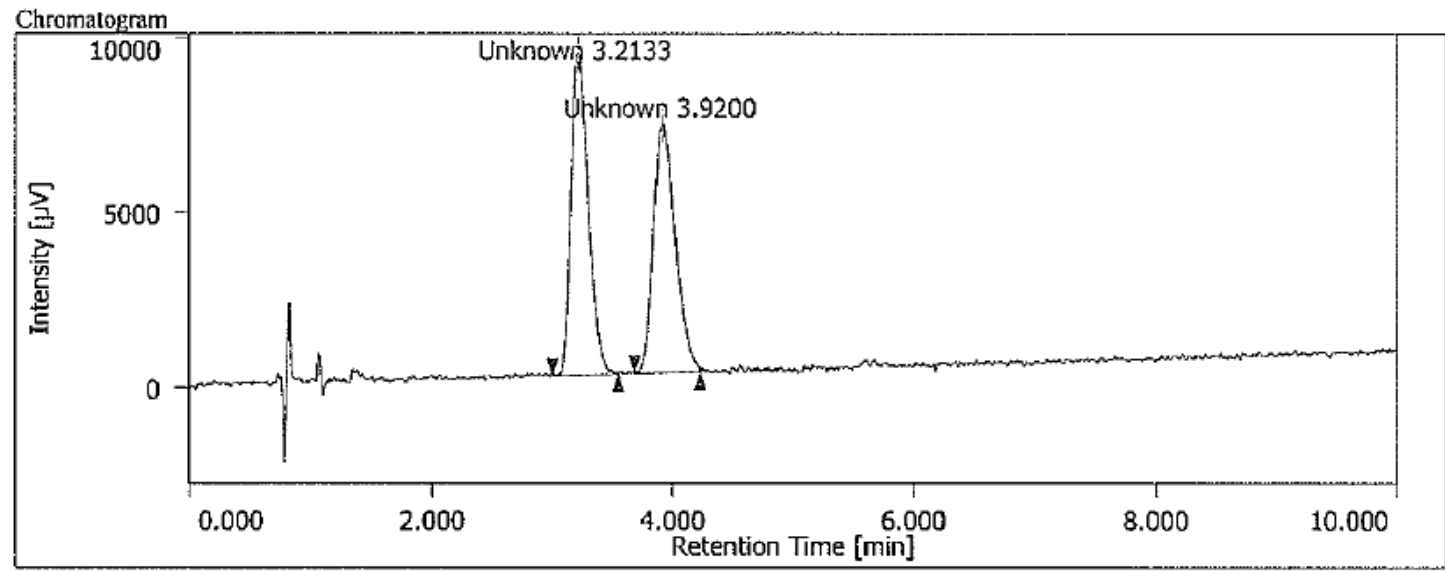

Peak Information

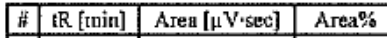

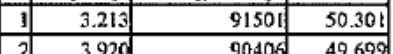

Figure S3. Enantiomeric mixture of $\mathbf{1 1}$.

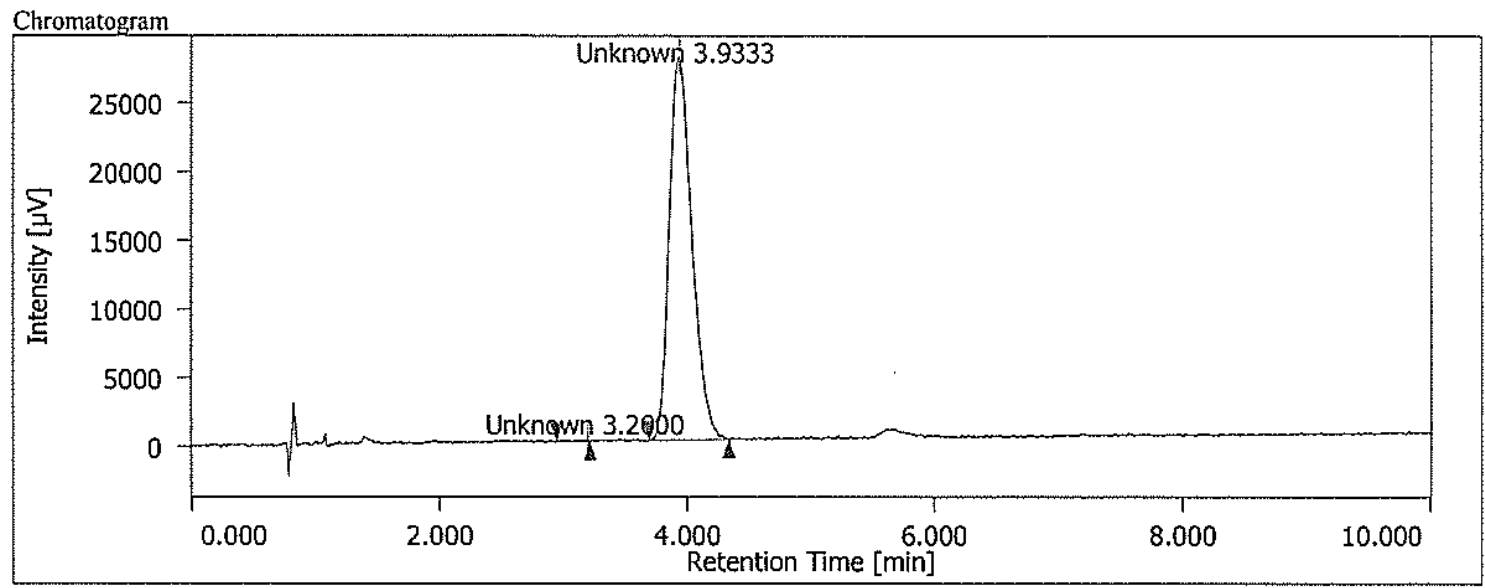

Peak Information

\begin{tabular}{|l|l|l|l|}
\hline$\#$ & tR [min] & Area $[\mu \mathrm{V} \cdot \mathrm{sec}]$ & Area\% \\
\hline & 3.200 & \\
\hline
\end{tabular}

\begin{tabular}{|r|r|r|r|}
\hline 1 & 3.200 & 714 & 0.202 \\
\hline 2 & 3.933 & 352707 & 99.798 \\
\hline
\end{tabular}

Figure S4. $(S)-11$. 


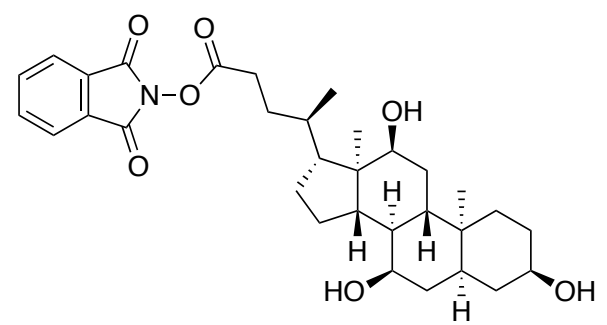

\section{1,3-dioxoisoindolin-2-yl $(R)-4-((3 R, 5 S, 7 R, 8 R, 9 S, 10 S, 12 S, 13 R, 14 S, 17 R)-3,7,12-t$ trihydroxy- 10,13-dimethylhexadecahydro- $1 H$-cyclopenta $[a]$ phenanthren-17-yl)pentanoate $(1 \mathrm{~m})$.}

The procedure was adapted from the method of Pratsch and coworkers. ${ }^{4}$ A round bottom flask containing a teflon-coated magnetic stir bar was charged with cholic acid $(4.09 \mathrm{~g}, 10.0 \mathrm{mmol}$, 1.00 equiv) and dry DMF $(100 \mathrm{~mL})$ under nitrogen. After sequential addition of $N$ hydroxyphthalimide (2.71 g, $16.6 \mathrm{mmol}, 1.66$ equiv), DMAP (244 mg, $2.00 \mathrm{mmol}, 0.200$ equiv), and $N, N^{\prime}$-diisopropylcarbodiimide $(2.33 \mathrm{~mL}, 15.0 \mathrm{mmol}, 1.50$ equiv), the reaction was allowed to stir overnight at room temperature. After this time, the heterogeneous mixture was filtered and the filtrate was concentrated under reduced pressure. The crude viscous liquid was diluted with hot methylcyclohexane and concentrated under reduced pressure. This process was repeated several times until a red solid formed. The solid was purified by silica gel chromatography $\left(100 \%\right.$ EtOAc, $\left.R_{f}=0.31\right)$ to yield the product as a colorless solid $(1.17 \mathrm{~g}, 2.12 \mathrm{mmol}, 21 \%$ yield).

${ }^{1} \mathrm{H}$ NMR (400 MHz; $\left.\mathrm{CDCl}_{3}\right): \delta 7.89(\mathrm{dd}, J=5.4,3.1 \mathrm{~Hz}, 2 \mathrm{H}), 7.79(\mathrm{dd}, J=5.5,3.1 \mathrm{~Hz}, 2 \mathrm{H})$, $4.01(\mathrm{~s}, 2 \mathrm{H}), 3.85(\mathrm{~s}, 2 \mathrm{H}), 3.49-3.43(\mathrm{~m}, 1 \mathrm{H}), 2.76-2.70(\mathrm{~m}, 1 \mathrm{H}), 2.66-2.60(\mathrm{~m}, 1 \mathrm{H}), 2.26-2.17$ $(\mathrm{m}, 2 \mathrm{H}), 1.35(\mathrm{dd}, J=40.6,27.6 \mathrm{~Hz}, 5 \mathrm{H}), 1.14$ (d, $J=6.5 \mathrm{~Hz}, 4 \mathrm{H}), 0.99-0.96(\mathrm{~m}, 2 \mathrm{H}), 0.90$ (s, $3 \mathrm{H}), 0.73(\mathrm{~s}, 3 \mathrm{H})$.

${ }^{13} \mathrm{C} \mathrm{NMR}\left(126 \mathrm{MHz}, \mathrm{CDCl}_{3}\right): \delta$ 170.2, $162.2134 .9,129.1,124.1,76.9,73.1,72.1,68.5,47.1$, 46.8, 42.4, 41.6, 39.9, 39.8, 35.4, 35.1, 34.8, 34.7, 30.8, 30.7, 28.6, 28.2, 27.6, 26.8, 23.3, 22.7, $17.5,12.7$.

mp $99-105^{\circ} \mathrm{C}$

IR ( $\left.\mathrm{cm}^{-1}\right): 3337,2932,2866,2361,1786,1740,1616,1466,1366,1184,1130,1076,972,910$, $876,729,698$.

LRMS (ESI+) $m / z: 576.3\left[\mathrm{M}+\mathrm{Na}^{+}\right]$. 


\section{Coupling of NHP Esters with Aryl Iodides}

General Procedure for the Coupling of $N$-(acyloxy)Phthalimides and Aryl Iodides.

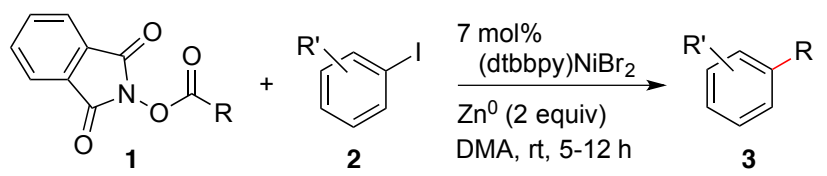

Reactions were set up in a nitrogen filled glove box. To an oven-dried 1-dram vial fitted with a Teflon-coated stir-bar was added (dtbbpy)NiBr 2 (27.3 mg, $0.0560 \mathrm{mmol}, 0.0700$ equiv), $N$ (acyloxy)phthalimide (1.20 mmol, 1.50 equiv), aryl iodide ( $0.800 \mathrm{mmol}, 1.00$ equiv), dodecane (as an internal standard, $10.0 \mu \mathrm{L}$ ), zinc flake (105 mg, $1.60 \mathrm{mmol}, 2.00$ equiv), and DMA (1.00 $\mathrm{mL}$ ). The vial was capped with a screw cap fitted with a PTFE-faced silicone septum, removed from the glove box, and heated in a reaction block set to $28{ }^{\circ} \mathrm{C}$ on the benchtop with stirring at $1195 \mathrm{rpm}$ until the reaction was judged complete $(<5 \% N$-(acyloxy)phthalimide or aryl iodide remaining) by GC analysis, typically $5-11 \mathrm{~h}$.

Alternatively, the catalyst could be generated by pre-stirring $\mathrm{NiBr}_{2}$ (diglyme) $(0.0560$ mmol, 0.0700 equiv) and 4-4'-di-tert-butyl-2,2'-bipyridine (15.0 mg, $0.0560 \mathrm{mmol}, 0.0700$ equiv) in DMA $(1.00 \mathrm{~mL})$ for $\sim 10 \mathrm{~min}$. The remaining reagents were then added, following the order of addition above.

\section{GC Analysis.}

After 5-11 h, the reaction was vented to an oil bubbler out before removing a $25 \mu \mathrm{L}$ aliquot of the reaction mixture with a $50 \mu \mathrm{L}$ gas-tight syringe. The aliquot was diluted with diethyl ether $(1.5 \mathrm{~mL})$ and filtered through a short silica pad $(1.5 \mathrm{~cm})$ in a pipette packed with glass wool. The filtrate was analyzed by gas chromatography and percent yield was calculated versus dodecane as the internal standard.

\section{Isolation and Purification.}

Upon reaction completion (judged as $<5 \% N$-(acyloxy)phthalimide or aryl iodide remaining by $\mathrm{GC}$ analysis) the reaction mixture was filtered through a short plug of silica gel $(1.5 \mathrm{~cm}$ wide $\times 2 \mathrm{~cm}$ high) to remove metal salts and eluted with diethyl ether $(75 \mathrm{~mL})$. DMA was removed by adding deionized water $(30 \mathrm{~mL})$ to the organic layer and extracting the aqueous layer with diethyl ether $(3 \times 30 \mathrm{~mL})$. The combined organic layers were dried over $\mathrm{MgSO}_{4}$, filtered, and concentrated under reduced pressure. The resulting residue was purified by column chromatography on silica gel to afford the pure product. 


\section{Large-Scale Procedure conducted on the benchtop in a round-bottom flask.}

To a $25 \mathrm{~mL}$ round-bottom flask containing a Teflon-coated stir-bar was added nickel pre-catalyst (87 $\mathrm{mg}$ of $\mathrm{NiBr}_{2}$ (diglyme), $0.28 \mathrm{mmol}$ ) and 4,4'-di-tert-butyl-2,2'-bipyridine (75 $\mathrm{mg}, 0.28$ mmol). The reaction flask was sealed with a rubber septum, and the septum was fitted with a needle connected to an oil bubbler and a needle connected to an argon manifold. The headspace of the flask was purged with argon gas for ten minutes and then the needle to the bubbler was removed. DMA $(5 \mathrm{~mL})$, was added via syringe and the resulting mixture was stirred on the benchtop ( 1200 rpm) at rt under Ar for 15 minutes to allow ligation of the nickel. The color of the reaction mixture became blue-green. Iodobenzene $(0.448 \mathrm{~mL}, 816 \mathrm{mg}, 4.00 \mathrm{mmol})$ was added via syringe, and 1,3-dioxoisoindolin-2-yl 3,3-dimethylbutanoate (1a) (1.57 g, $6.00 \mathrm{mmol})$ and zinc dust $(0.52 \mathrm{~g}, 8.0 \mathrm{mmol})$ were added in one portion to the reaction vessel by quickly removing the septum and replacing it. The reaction mixture was warmed to $28{ }^{\circ} \mathrm{C}$ in an oil bath and stirred at that temperature under Ar. After the reaction was judged complete by GC analysis $(\sim 12 \mathrm{~h}$ ), the reaction mixture was filtered through a plug of silica. The pad was washed with $\mathrm{Et}_{2} \mathrm{O}(100 \mathrm{~mL})$ and concentrated. The resulting residue was purified by chromatography on $\mathrm{SiO}_{2}$ (pentane) to yield $480 \mathrm{mg}$ of 3a ( $81 \%$ yield). Characterization data matched those obtained on $0.8 \mathrm{mmol}$ scale (vide infra).

\section{Product Characterization}

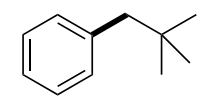

\section{t-butylmethylbenzene [1007-26-7] (3a).}

The general procedure was followed with (dtbbpy) $\mathrm{NiBr}_{2}(27.3 \mathrm{mg}, 0.0560 \mathrm{mmol}, 0.0700$ equiv), 1,3-dioxoisoindolin-2-yl 3,3-dimethylbutanoate (314 mg, $1.20 \mathrm{mmol}, 1.50$ equiv), and iodobenzene $(89.5 \mu \mathrm{L}, 0.800 \mathrm{mmol}, 1.00$ equiv). After $5 \mathrm{~h}$, the reaction mixture was worked up and purified by flash chromatography $\left(100 \%\right.$ pentane, $\left.\mathrm{R}_{\mathrm{f}}=0.54\right)$ to afford a colorless oil (103 $\mathrm{mg}, 87 \%$ yield). Analytical data matched those in the literature. ${ }^{8}$

${ }^{1} \mathrm{H}$ NMR (500 MHz; $\mathrm{CDCl}_{3}$ ): $\delta$ 7.28-7.12 (m, 5H), 2.50 (s, 2H), 0.91 (s, 9H).

${ }^{13} \mathrm{C} \mathrm{NMR}\left(126 \mathrm{MHz}, \mathrm{CDCl}_{3}\right): \delta 139.9,130.6,127.7,125.8,50.4,31.9,29.5$.

GC-MS m/z (\% relative intensity, ion): $149.10\left(2.32, \mathrm{M}^{+}\right), 133.10\left(15.93, \mathrm{M}^{+}-\mathrm{CH}_{3}\right), 92.10$ $\left(100.0, \mathrm{M}^{+}-\mathrm{C}_{4} \mathrm{H}_{8}\right), 57.10\left(89.08, \mathrm{M}^{+}-\mathrm{C}_{7} \mathrm{H}_{7}\right)$.

\footnotetext{
${ }^{8}$ Everson, D. A.; Shrestha, R.; Weix, D. J. J. Am. Chem. Soc. 2010, 132, 920-921.
} 
<smiles>c1ccc(CCCc2ccccc2)cc1</smiles>

\section{1,3-diphenylpropane [1081-75-0] (3b).}

The general procedure was followed with $\mathrm{NiBr}_{2}$ (diglyme) $(17.7 \mathrm{mg}, 0.0560 \mathrm{mmol}, 0.0700$ equiv), 4-4'-di-tert-butyl-2,2'-bipyridine $(15.0 \mathrm{mg}, 0.0560 \mathrm{mmol}, 0.0700$ equiv), 1,3dioxoisoindolin-2-yl 4-phenylbutanoate $(247.5 \mathrm{mg}, 0.800 \mathrm{mmol}, 1.00$ equiv), and iodobenzene $(89.5 \mu \mathrm{L}, 0.800 \mathrm{mmol}, 1.00$ equiv). After $11 \mathrm{~h}$, the reaction mixture was worked up and purified by flash chromatography $\left(100 \%\right.$ pentane, $\left.\mathrm{R}_{\mathrm{f}}=0.50\right)$ to afford a colorless oil (152 $\mathrm{mg}, 97 \%$ yield). Analytical data matched those in the literature. ${ }^{9}$

${ }^{1} \mathrm{H}$ NMR (400 MHz; $\left.\mathrm{CDCl}_{3}\right): \delta 7.31-7.26(\mathrm{~m}, 4 \mathrm{H}), 7.20(\mathrm{~d}, J=6.4 \mathrm{~Hz}, 6 \mathrm{H}), 2.67$ (t, $J=7.7 \mathrm{~Hz}$, $4 \mathrm{H}$ ), 1.98 (quintet, $J=7.7 \mathrm{~Hz}, 2 \mathrm{H}$ ).

${ }^{13} \mathrm{C}$ NMR (126 MHz, $\left.\mathrm{CDCl}_{3}\right): \delta 142.4,128.6,128.4,125.9,35.6,33.1$.

GC-MS m/z (\% relative intensity, ion): $197.20\left(12.21, \mathrm{M}^{+}\right), 92.15\left(100.00, \mathrm{M}^{+}-\mathrm{C}_{8} \mathrm{H}_{9}\right), 77.05$

(12.58, $\left.\mathrm{M}^{+}-\mathrm{C}_{9} \mathrm{H}_{14}\right)$.

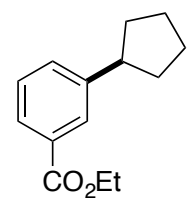

\section{3-cyclopentyl-benzoic acid ethyl ester [1027294-28-5] (3c).}

The general procedure was followed with $\mathrm{NiBr}_{2}$ (diglyme) $(17.7 \mathrm{mg}, 0.0560 \mathrm{mmol}, 0.0700$ equiv), 4-4'-di-tert-butyl-2,2'-bipyridine $(15.0 \mathrm{mg}, 0.0560 \mathrm{mmol}, 0.0700$ equiv), 1,3dioxoisoindolin-2-yl cyclopentanecarboxylate (311 mg, $1.20 \mathrm{mmol}, 1.50$ equiv), and ethyl 3iodobenzoate (135 $\mu \mathrm{L}, 0.800 \mathrm{mmol}, 1.00$ equiv). After $11 \mathrm{~h}$, the reaction mixture was filtered through a silica plug with diethyl ether, concentrated under reduced pressure, and purified by flash chromatography $\left(2 \% \mathrm{Et}_{2} \mathrm{O}\right.$ in pentane, $\left.\mathrm{R}_{\mathrm{f}}=0.31\right)$ to afford a pale yellow oil $(155 \mathrm{mg}, 89 \%$ yield). Analytical data matched those in the literature. ${ }^{10}$

${ }^{1} \mathrm{H}$ NMR (400 MHz; $\left.\mathrm{CDCl}_{3}\right): \delta 7.92(\mathrm{~s}, 1 \mathrm{H}), 7.85(\mathrm{~d}, J=7.6 \mathrm{~Hz}, 1 \mathrm{H}), 7.43(\mathrm{~d}, J=7.7 \mathrm{~Hz}, 1 \mathrm{H})$, $7.34(\mathrm{t}, J=7.6 \mathrm{~Hz}, 1 \mathrm{H}), 4.38(\mathrm{q}, J=7.1 \mathrm{~Hz}, 2 \mathrm{H}), 3.04$ (quintet, $J=8.6 \mathrm{~Hz}, 1 \mathrm{H}), 2.13-2.06(\mathrm{~m}$, $2 \mathrm{H}), 1.87-1.77(\mathrm{~m}, 2 \mathrm{H}), 1.70(\mathrm{dd}, J=12.4,7.8 \mathrm{~Hz}, 2 \mathrm{H}), 1.66-1.57(\mathrm{~m}, 2 \mathrm{H}), 1.40(\mathrm{t}, J=7.1 \mathrm{~Hz}$, $3 \mathrm{H})$.

${ }^{13} \mathrm{C} \mathrm{NMR}\left(126 \mathrm{MHz}, \mathrm{CDCl}_{3}\right): \delta 167.1,147.0,131.8,130.6,129.7,128.4,127.1,61.0,45.9,34.8$, $25.7,14.5$.

\footnotetext{
${ }^{9}$ Gieshoff, T. M.; Villa, M.; Welther, A.; Polis, M.; Chakraborty, U.; Wolf, R.; von Wangelin, A. J. Green Chem. 2015, 17, 1408-1413.

${ }^{10}$ Cuisiat, S.; Bourdiol, N.; Lacharme, V.; Newman-Tancredi, A.; Colpaert, F.; Vacher, B. J. Med. Chem. 2007, 50, 865-876.
} 
GC-MS m/z (\% relative intensity, ion): $219.15\left(12.11, \mathrm{M}^{+}\right), 189.10\left(56.46, \mathrm{M}^{+}-\mathrm{C}_{2} \mathrm{H}_{5}\right), 173.15$ (59.21, $\left.\mathrm{M}^{+}-\mathrm{C}_{2} \mathrm{H}_{5} \mathrm{O}\right), 145.15\left(100.00, \mathrm{M}^{+}-\mathrm{C}_{3} \mathrm{H}_{5} \mathrm{O}_{2}\right), 117.10\left(73.46, \mathrm{M}^{+}-\mathrm{C}_{5} \mathrm{H}_{9} \mathrm{O}_{2}\right), 69.05\left(12.00, \mathrm{M}^{+}-\right.$ $\left.\mathrm{C}_{9} \mathrm{H}_{9} \mathrm{O}_{2}\right)$.

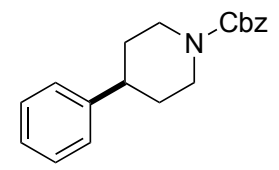

\section{4-phenylpiperidine-1-carboxylic acid benzyl ester [733810-73-6] (3d).}

The general procedure was followed with $\mathrm{NiBr}_{2}$ (diglyme) (15.2 mg, $0.0480 \mathrm{mmol}, 0.120$ equiv), 4-4'-di-tert-butyl-2,2'-bipyridine (12.9 mg, $0.0480 \mathrm{mmol}, 0.120$ equiv), 1-benzyl 4-(1,3dioxoisoindolin-2-yl) piperidine-1,4-dicarboxylate (163 mg, $0.400 \mathrm{mmol}, 1.00$ equiv), iodobenzene (44.8 $\mu \mathrm{L}, 0.400 \mathrm{mmol}, 1.00$ equiv), $\mathrm{Zn}$ (52.3 mg, $0.800 \mathrm{mmol}, 2.00$ equiv), and DMA $(0.500 \mathrm{~mL})$. After $9 \mathrm{~h}$, the reaction mixture was filtered through a silica plug with diethyl ether, concentrated under reduced pressure, and purified by flash chromatography (90:10, hexanes/acetone, $\left.\mathrm{R}_{\mathrm{f}}=0.31\right)$ to afford a white solid $(75.4 \mathrm{mg}, 64 \%$ yield). Analytical data matched those in the literature. ${ }^{11}$

${ }^{1} \mathrm{H}$ NMR (500 MHz; $\mathrm{CDCl}_{3}$ ): $\delta$ 7.38-7.29 (m, 7H), 7.20 (dd, $\left.J=12.7,7.4 \mathrm{~Hz}, 3 \mathrm{H}\right), 5.16(\mathrm{~s}, 2 \mathrm{H})$, 4.34 (s, 2H), 2.89 (s, 2H), 2.67 (t, $J=12.2 \mathrm{~Hz}, 1 \mathrm{H}), 1.84$ (d, $J=11.6 \mathrm{~Hz}, 2 \mathrm{H}), 1.65$ (d, $J=10.6$ $\mathrm{Hz}, 2 \mathrm{H})$.

${ }^{13} \mathrm{C} \mathrm{NMR}\left(126 \mathrm{MHz}, \mathrm{CDCl}_{3}\right): \delta 155.4,145.7,137.2,128.7,128.6,128.1,128.0,126.9,126.6$, 67.2, 44.8, 42.8, 33.3.

mp $56-57{ }^{\circ} \mathrm{C}$

IR ( $\left.\mathrm{cm}^{-1}\right): 3028,2936,2851,2361,1682,1493,1431,1362,1277,1223,1123,1080,1015,748$, 698.

LRMS (ESI+) $m / z: 431.2\left[\mathrm{M}+\mathrm{Na}^{+}\right], 447.1\left[\mathrm{M}+\mathrm{K}^{+}\right]$.

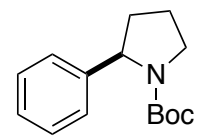

\section{N-(tert-butoxycarbonyl)-2-phenylpyrrolidine [154874-88-1] (3e).}

The general procedure was followed with $\mathrm{NiBr}_{2}$ (diglyme) (14.9 mg, $0.0480 \mathrm{mmol}, 0.120$ equiv), 4-4'-di-tert-butyl-2,2'-bipyridine (12.9 mg, $0.0480 \mathrm{mmol}, 0.120$ equiv), 1-(tert-butyl) 2-(1,3dioxoisoindolin-2-yl) pyrrolidine-1,2-dicarboxylate $(216 \mathrm{mg}, 0.600 \mathrm{mmol}, 1.50$ equiv), iodobenzene (44.8 $\mu \mathrm{L}, 0.400 \mathrm{mmol}, 1.00$ equiv), $\mathrm{Zn}$ (52.3 mg, $0.800 \mathrm{mmol}, 2.00$ equiv), and DMA $(0.500 \mathrm{~mL})$. After $8 \mathrm{~h}$, the reaction mixture was filtered through a silica plug with diethyl

\footnotetext{
${ }^{11}$ Powell, D. A.; Fu, G. C. J. Am. Chem. Soc. 2004, 126, 7788-7789.
} 
ether, concentrated under reduced pressure, and purified by flash chromatography (90:10, hexanes/acetone, $\left.\mathrm{R}_{\mathrm{f}}=0.31\right)$ to afford a white solid $(66.5 \mathrm{mg}, 67 \%$ yield $)$. The isolated product was contaminated with a small amount of $N$-Boc-pyrrolidine. The actual yield of product was determined to be $63.8 \mathrm{mg}$ (65\% yield). Analytical data matched those in the literature. ${ }^{12}$

Due to the existence of rotamers at ambient temperature, the ${ }^{1} \mathrm{H}$ NMR spectrum was obtained at $55^{\circ} \mathrm{C}$.

${ }^{1} \mathrm{H}$ NMR (500 MHz; $\left.\mathrm{CDCl}_{3 ;} 55^{\circ} \mathrm{C}\right): \delta 7.26(\mathrm{q}, J=6.5 \mathrm{~Hz}, 2 \mathrm{H}), 7.19-7.14(\mathrm{~m}, 3 \mathrm{H}), 4.82(\mathrm{~s}, 1 \mathrm{H})$, $3.59(\mathrm{t}, J=6.3 \mathrm{~Hz}, 2 \mathrm{H}), 2.32-2.26(\mathrm{~m}, 1 \mathrm{H}), 1.95-1.80(\mathrm{~m}, 3 \mathrm{H}), 1.27(\mathrm{~s}, 9 \mathrm{H})$.

${ }^{13} \mathrm{C} \mathrm{NMR}\left(126 \mathrm{MHz}, \mathrm{CDCl}_{3}\right): \delta 154.7,145.3,128.2,126.6,125.6,79.3,61.5,47.2,36.2,28.3$, 23.3 .

mp 47-49 ${ }^{\circ} \mathrm{C}\left(\right.$ lit. $\left.^{13} \mathrm{mp} 62-63{ }^{\circ} \mathrm{C}\right)$

IR (cm $\left.{ }^{-1}\right): 2970,2886,2158,2021,1975,1678,1449,1398,1362,1256,1165,1155,1113,876$, 764, 737, 700.

LRMS (ESI+) $m / z: 270.2\left[\mathrm{M}+\mathrm{Na}^{+}\right]$.

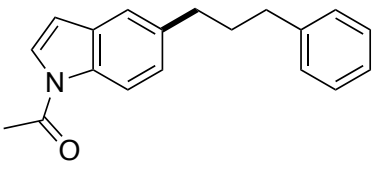

\section{1-(5-(3-phenylpropyl)-1H-indol-1-yl)ethan-1-one (3f).}

The general procedure was followed with $\mathrm{NiBr}_{2}$ (diglyme) $(17.7 \mathrm{mg}, 0.0560 \mathrm{mmol}, 0.0700$ equiv), 4-4'-di-tert-butyl-2,2'-bipyridine $(15.0 \mathrm{mg}, \quad 0.0560 \mathrm{mmol}, 0.0700$ equiv), 1,3dioxoisoindolin-2-yl 4-phenylbutanoate $(371 \mathrm{mg}, 1.20 \mathrm{mmol}, 1.50$ equiv), and 1-(5-iodo-1Hindol-1-yl)ethanone (228 mg, $0.800 \mathrm{mmol}, 1.00$ equiv). After $8 \mathrm{~h}$, the reaction mixture was worked up and purified by flash chromatography $\left(85: 15\right.$, hexanes/acetone, $\left.\mathrm{R}_{\mathrm{f}}=0.33\right)$ to afford a light yellow oil (198 mg, 89\% yield).

${ }^{1} \mathrm{H}$ NMR (500 MHz; $\left.\mathrm{CDCl}_{3}\right): \delta 8.32(\mathrm{~d}, J=7.0 \mathrm{~Hz}, 1 \mathrm{H}), 7.37(\mathrm{~d}, J=13.6 \mathrm{~Hz}, 2 \mathrm{H}), 7.27(\mathrm{dd}, J=$ 13.4, $6.3 \mathrm{~Hz}, 2 \mathrm{H}), 7.18(\mathrm{t}, J=7.2 \mathrm{~Hz}, 4 \mathrm{H}), 6.58(\mathrm{~d}, J=3.7 \mathrm{~Hz}, 1 \mathrm{H}), 2.75(\mathrm{t}, J=7.6 \mathrm{~Hz}, 2 \mathrm{H}), 2.66$ (t, $J=7.8 \mathrm{~Hz}, 2 \mathrm{H}), 2.62$ (s, 3H), 2.00 (quintet, $J=7.7 \mathrm{~Hz}, 2 \mathrm{H}$ ).

${ }^{13} \mathrm{C} \mathrm{NMR}\left(126 \mathrm{MHz}, \mathrm{CDCl}_{3}\right): \delta 168.6,142.5,137.9,134.1,130.8,128.6,128.4,126.0,125.8$, 125.4, 120.4, 116.4, 109.2, 35.5, 35.5, 33.5, 24.0.

IR ( $\left.\mathrm{cm}^{-1}\right): 3387,3340,2936,2866,2361,1813,1786,1740,1466,1377,1327,1076,910,880$, 729, 698.

LRMS (ESI+) $m / z: 278.2\left[\mathrm{M}+\mathrm{H}^{+}\right], 300.2\left[\mathrm{M}+\mathrm{Na}^{+}\right]$.

\footnotetext{
${ }^{12}$ Beak, P.; Wu, S.; Yum, E. K.; Jun, Y. M. J. Org. Chem. 1994, 59, 276-277., Dieter, R. K.; Li, S. J. Org. Chem. 1997, 62, 7726-7735.

${ }^{13}$ Barker, G.; O’Brien, P.; Campos, K. R. Org. Lett. 2010, 12 , 4176-4179
} 


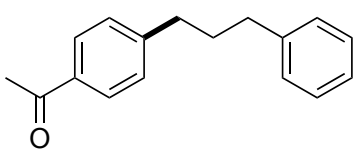

\section{1-(p-Acetylphenyl)-3-phenylpropane [113138-08-2] (3g).}

The general procedure was followed with (dtbbpy) $\mathrm{NiBr}_{2}(27.3 \mathrm{mg}, 0.0560 \mathrm{mmol}, 0.0700$ equiv), 1,3-dioxoisoindolin-2-yl 4-phenylbutanoate (371 mg, $1.20 \mathrm{mmol}, 1.50$ equiv), and 4iodoacetophenone $(197 \mu \mathrm{L}, 0.800 \mathrm{mmol}, 1.00$ equiv). After $5 \mathrm{~h}$, the reaction mixture was worked up and purified by flash chromatography $\left(95: 5\right.$ hexanes/acetone, $\left.\mathrm{R}_{\mathrm{f}}=0.14\right)$ to afford a colorless oil (135 mg, 71\% yield). Analytical data matched those in the literature. ${ }^{14}$

${ }^{1} \mathrm{H} \mathrm{NMR}\left(400 \mathrm{MHz} ; \mathrm{CDCl}_{3}\right): \delta 7.88(\mathrm{~d}, J=8.2 \mathrm{~Hz}, 2 \mathrm{H}), 7.31-7.26(\mathrm{~m}, 4 \mathrm{H}), 7.19(\mathrm{t}, J=8.4 \mathrm{~Hz}$, $3 \mathrm{H}$ ), 2.68 (dt, $J=20.3,7.7 \mathrm{~Hz}, 4 \mathrm{H}), 2.58(\mathrm{~s}, 3 \mathrm{H}), 1.98$ (quintet, $J=7.7 \mathrm{~Hz}, 2 \mathrm{H}$ ).

${ }^{13} \mathrm{C} \mathrm{NMR}\left(126 \mathrm{MHz}, \mathrm{CDCl}_{3}\right): \delta 197.9,148.2,142.0,135.2,128.8,128.6,128.5,128.5,126.0$, $35.5,35.5,32.7,26.7$.

GC-MS m/z (\% relative intensity, ion): $238.10\left(70.99, \mathrm{M}^{+}\right), 223.05\left(100.00, \mathrm{M}^{+}-\mathrm{CH}_{3}\right), 147.05$ (75.15, $\left.\mathrm{M}^{+}-\mathrm{C}_{7} \mathrm{H}_{7}\right), 134.05$ (84.36), 105.05 (94.62, $\left.\mathrm{M}^{+}-\mathrm{C}_{9} \mathrm{H}_{9} \mathrm{O}\right)$.

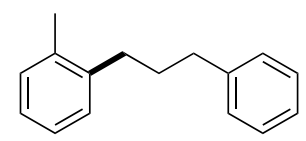

\section{1-methyl-2-(3-phenylpropyl)benzene [1520-39-4] (3h).}

The general procedure was followed with $\mathrm{NiBr}_{2}$ (diglyme) $(17.7 \mathrm{mg}, 0.0560 \mathrm{mmol}, 0.0700$ equiv), 4-4'-di-tert-butyl-2,2'-bipyridine (15.0 mg, $0.0560 \mathrm{mmol}, 0.0700$ equiv), 1,3dioxoisoindolin-2-yl 4-phenylbutanoate (248 mg, $0.800 \mathrm{mmol}, 1.00$ equiv), and 2-iodotoluene (102 $\mu \mathrm{L}, 0.800 \mathrm{mmol}, 1.00$ equiv). After $4 \mathrm{~h}$, the reaction mixture was filtered through a silica plug with diethyl ether, concentrated under reduced pressure, and purified by flash chromatography $\left(100 \%\right.$ pentane, $\left.\mathrm{R}_{\mathrm{f}}=0.47\right)$. Mixed fractions of the desired product were obtained. The mixed fractions were combined, concentrated under reduced pressure and the residue was resubjected to flash chromatography (100\% pentane) to afford a colorless oil (153 $\mathrm{mg}, 91 \%$ yield). Analytical data matched those in the literature. ${ }^{15}$

${ }^{1} \mathrm{H}$ NMR (500 MHz; $\mathrm{CDCl}_{3}$ ): $\delta$ 7.31-7.25 (m, 3H), 7.20 (dt, $\left.J=8.8,4.7 \mathrm{~Hz}, 3 \mathrm{H}\right), 7.14-7.10(\mathrm{~m}$, 4H), $2.71(\mathrm{t}, J=7.7 \mathrm{~Hz}, 2 \mathrm{H}), 2.64(\mathrm{t}, J=7.9 \mathrm{~Hz}, 2 \mathrm{H}), 2.27$ (s, 3H), 1.92 (quintet, $J=7.8 \mathrm{~Hz}$, $2 \mathrm{H})$.

${ }^{13} \mathrm{C} \mathrm{NMR}\left(126 \mathrm{MHz}, \mathrm{CDCl}_{3}\right): \delta 142.4,140.6,136.0,130.3,128.9,128.6,128.5,128.4,126.0$, $126.0,125.9,36.0,33.0,31.9,19.4$.

\footnotetext{
${ }^{14}$ Molander, G.; Ito, T. Org. Lett. 2001, 3, 393-396.

${ }^{15}$ Moriya, T.; Kentaro, T.; Konakahara, T.; Ogiaware, Y.; Sakai, N. Eur, J. Org. Chem. 2015, 10, $2277-2281$.
} 
GC-MS m/z (\% relative intensity, ion): $211.15\left(8.35, \mathrm{M}^{+}\right), 119.10\left(53.90, \mathrm{M}^{+}-\mathrm{C}_{7} \mathrm{H}_{7}\right), 106.10$ $\left(100.00, \mathrm{M}^{+}-\mathrm{C}_{8} \mathrm{H}_{9}\right), 91.10\left(47.45, \mathrm{M}^{+}-\mathrm{C}_{7} \mathrm{H}_{7}\right)$.

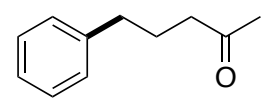

\section{5-phenyl-2-pentanone [2235-83-8] (3i).}

The general procedure was followed with $\mathrm{NiBr}_{2}$ (diglyme) $(17.7 \mathrm{mg}, 0.0560 \mathrm{mmol}, 0.0700$ equiv), 4-4'-di-tert-butyl-2,2'-bipyridine $(15.0 \mathrm{mg}, \quad 0.0560 \mathrm{mmol}, 0.0700$ equiv), 1,3dioxoisoindolin-2-yl 5-oxohexanoate (330 mg, $1.20 \mathrm{mmol}, 1.50$ equiv), and iodobenzene (89.5 $\mu \mathrm{L}, 0.800 \mathrm{mmol}, 1.00$ equiv). After $14 \mathrm{~h}$, the reaction mixture was filtered through a silica plug with diethyl ether, concentrated under reduced pressure, and purified by flash chromatography (20:1 Pentane/ $\left.\mathrm{Et}_{2} \mathrm{O}, \mathrm{R}_{\mathrm{f}}=0.18\right)$ to afford a light yellow oil $(90.9 \mathrm{mg}, 70 \%$ yield). Analytical data matched those in the literature. ${ }^{16}$

${ }^{1} \mathrm{H}$ NMR (400 MHz; $\left.\mathrm{CDCl}_{3}\right): \delta 7.28(\mathrm{dd}, J=12.6,5.1 \mathrm{~Hz}, 3 \mathrm{H}), 7.19(\mathrm{dd}, J=12.4,7.1 \mathrm{~Hz}, 3 \mathrm{H})$, $2.63(\mathrm{t}, J=7.6 \mathrm{~Hz}, 2 \mathrm{H}), 2.44(\mathrm{t}, J=7.4 \mathrm{~Hz}, 2 \mathrm{H}), 2.12(\mathrm{~s}, 3 \mathrm{H}), 1.91$ (quintet, $J=7.5 \mathrm{~Hz}, 2 \mathrm{H}$ ).

${ }^{13} \mathrm{C} \mathrm{NMR}\left(126 \mathrm{MHz}, \mathrm{CDCl}_{3}\right): \delta 208.8,141.7,128.6,128.5,126.0,42.9,35.1,30.0,25.3$. GC-MS m/z (\% relative intensity, ion): $163.15\left(8.31, \mathrm{M}^{+}\right), 104.10\left(100.00, \mathrm{M}^{+}-\mathrm{C}_{3} \mathrm{H}_{5} \mathrm{O}\right), 91.10$ $\left(14.71, \mathrm{M}^{+}-\mathrm{C}_{7} \mathrm{H}_{7}\right)$.

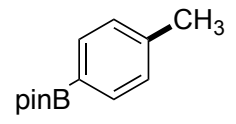

\section{4,4,5,5-tetramethyl-2-(p-tolyl)-1,3,2-dioxaborolane [195062-57-8] (3j).}

The general procedure was followed with $\mathrm{NiBr}_{2}$ (diglyme) $(17.7 \mathrm{mg}, 0.0560 \mathrm{mmol}, 0.0700$ equiv), 4-4'-di-tert-butyl-2,2'-bipyridine $(15.0 \mathrm{mg}, 0.0560 \mathrm{mmol}, 0.0700$ equiv), 1,3dioxoisoindolin-2-yl acetate (246 mg, $1.20 \mathrm{mmol}, 1.50$ equiv), and 4-iodophenylboronic acid pinacol ester (264 mg, $0.800 \mathrm{mmol}, 1.00$ equiv). After $6 \mathrm{~h}$, the reaction mixture was worked up and purified by flash chromatography $\left(97: 3\right.$ hexanes/acetone, $\left.\mathrm{R}_{\mathrm{f}}=0.30\right)$ to afford a white solid (72.2 mg, 41\% yield). The actual yield of the reaction was higher, but $\sim 19 \mathrm{mg}$ (11\%) product was collected in fractions that also contained the hydrodehalogenation byproduct, 4,4,5,5tetramethyl-2-phenyl-1,3,2-dioxaborolane and we were unable to separate this mixture further after repeated attempts. Analytical data matched those in the literature. ${ }^{17} \mathrm{In}$ the ${ }^{13} \mathrm{C}$ NMR spectrum, the carbon attached to boron was not observed. ${ }^{18}$

\footnotetext{
${ }^{16}$ Moriyama, K.; Takemura, M.; Togo, H. J. Org. Chem. 2014, 79, 6094-6104.

${ }^{17}$ Mo, F.; Jiang, Y.; Qiu, D.; Zhang, Y.; Wang, J. Angew. Chem. Int. Ed. 2010, 49, 1846-1849.

${ }^{18}$ Zhao, C.-J.; Xue, D.; Jia, Z.-H.; Wang, C.; Xiao, J. Synlett. 2014, 25, 1577-1584.
} 
${ }^{1} \mathrm{H} \mathrm{NMR}\left(400 \mathrm{MHz} ; \mathrm{CDCl}_{3}\right): \delta 7.70(\mathrm{~d}, J=7.8 \mathrm{~Hz}, 2 \mathrm{H}), 7.19(\mathrm{~d}, J=7.6 \mathrm{~Hz}, 2 \mathrm{H}), 2.36(\mathrm{~s}, 3 \mathrm{H})$, $1.34(\mathrm{~s}, 12 \mathrm{H})$.

${ }^{13} \mathrm{C}$ NMR (126 MHz, $\left.\mathrm{CDCl}_{3}\right): \delta 141.5,134.9,128.7,83.7,25.0,21.9$.

mp $48-49^{\circ} \mathrm{C}$ (lit. $\left.{ }^{19} \mathrm{mp} 47.2-48.0^{\circ} \mathrm{C}\right)$

IR $\left(\mathrm{cm}^{-1}\right): 2976,2924,2158,2021,1977,1610,1439,1396,1358,1319,1265,1213,1142,1088$, $1022,962,858,816,725,700$.

LRMS (ESI+) $m / z: 219.2\left[\mathrm{M}+\mathrm{H}^{+}\right], 241.2\left[\mathrm{M}+\mathrm{Na}^{+}\right]$.

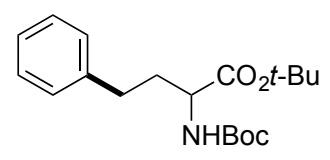

(S)-tert-butyl 2-((tert-butoxycarbonyl)amino)-4-phenylbutanoate [111652-08-5] (3k).

The general procedure was followed with $\mathrm{NiBr}_{2}$ (diglyme) $(17.7 \mathrm{mg}, 0.0560 \mathrm{mmol}, 0.0700$ equiv), 4-4'-di-tert-butyl-2,2'-bipyridine ( $15.0 \mathrm{mg}, 0.0560 \mathrm{mmol}, 0.0700$ equiv), 1-(tert-butyl) 5 (1,3-dioxoisoindolin-2-yl) (tert-butoxycarbonyl)-glutamate (538 mg, $1.20 \mathrm{mmol}, 1.50$ equiv), and iodobenzene $(89.5 \mu \mathrm{L}, 0.800 \mathrm{mmol}, 1.00$ equiv). After $14 \mathrm{~h}$, the reaction mixture was filtered through a silica plug with diethyl ether, concentrated under reduced pressure, and purified by flash chromatography (95:5, hexanes/acetone, $\left.R_{\mathrm{f}}=0.31\right)$ to afford a white solid (75.4 $\mathrm{mg}, 64 \%$ yield). Analytical data matched those in the literature. ${ }^{20}$

$[\alpha]_{\mathrm{D}}^{23}+30.3\left(\mathrm{c}=1.03, \mathrm{CHCl}_{3}\right)$

${ }^{1} \mathrm{H}$ NMR (400 MHz; $\left.\mathrm{CDCl}_{3}\right): \delta 7.29(\mathrm{~d}, J=7.5 \mathrm{~Hz}, 2 \mathrm{H}), 7.19(\mathrm{t}, J=8.0 \mathrm{~Hz}, 3 \mathrm{H}), 5.07$ (d, $J=7.3$ $\mathrm{Hz}, 1 \mathrm{H}), 4.25(\mathrm{~d}, J=5.5 \mathrm{~Hz}, 1 \mathrm{H}), 2.73-2.59(\mathrm{~m}, 2 \mathrm{H}), 2.10(\mathrm{dt}, J=11.0,5.9 \mathrm{~Hz}, 1 \mathrm{H}), 1.90(\mathrm{tt}, J=$ $11.9,6.0 \mathrm{~Hz}, 1 \mathrm{H}), 1.47(\mathrm{~d}, J=8.9 \mathrm{~Hz}, 18 \mathrm{H})$.

${ }^{13} \mathrm{C}$ NMR (126 MHz, $\left.\mathrm{CDCl}_{3}\right): \delta 171.9,155.5,141.3,128.6,128.5,126.2,82.0,79.7,54.0,34.9$, $31.7,28.4,28.1$.

mp 71-72 ${ }^{\circ} \mathrm{C}$ (lit. ${ }^{20} \mathrm{mp} 68-69^{\circ} \mathrm{C}$ ) IR $\left(\mathrm{cm}^{-1}\right)$ : 3395, 2974, 2927, 2361, 1728, 1694, 1497, 1362, 1150, 1030, 860, 845, 721, 698. LRMS (ESI+) $m / z: 336.3\left[\mathrm{M}+\mathrm{H}^{+}\right], 358.3\left[\mathrm{M}+\mathrm{Na}^{+}\right], 374.2\left[\mathrm{M}+\mathrm{K}^{+}\right]$.

SFC analysis (Daicel Chiralpak IA $(0.46 \mathrm{~cm} \mathrm{ID} \times 25 \mathrm{~cm} \mathrm{~L}), 10 \% i-\mathrm{PrOH}$ in $\mathrm{CO}_{2}, 4 \mathrm{~mL} / \mathrm{min}, \lambda=$ $\left.220 \mathrm{~nm}, 35^{\circ} \mathrm{C}, 12.0 \mathrm{MPa}\right)$ : tR $[\mathrm{min}]=2.3(1.4 \%), 2.7(98.6 \%), 97 \%$ ee.

\footnotetext{
${ }^{19}$ Labre, F.; Gimbert, Y.; Bannwarth, P.; Olivero, S.; Duñach, E.; Chavant, P. Y. Org. Lett. 2014, 16, 2366-2369.

${ }^{20}$ Münster, P.; Steglich, W. Synthesis 1987, 223-225.
} 


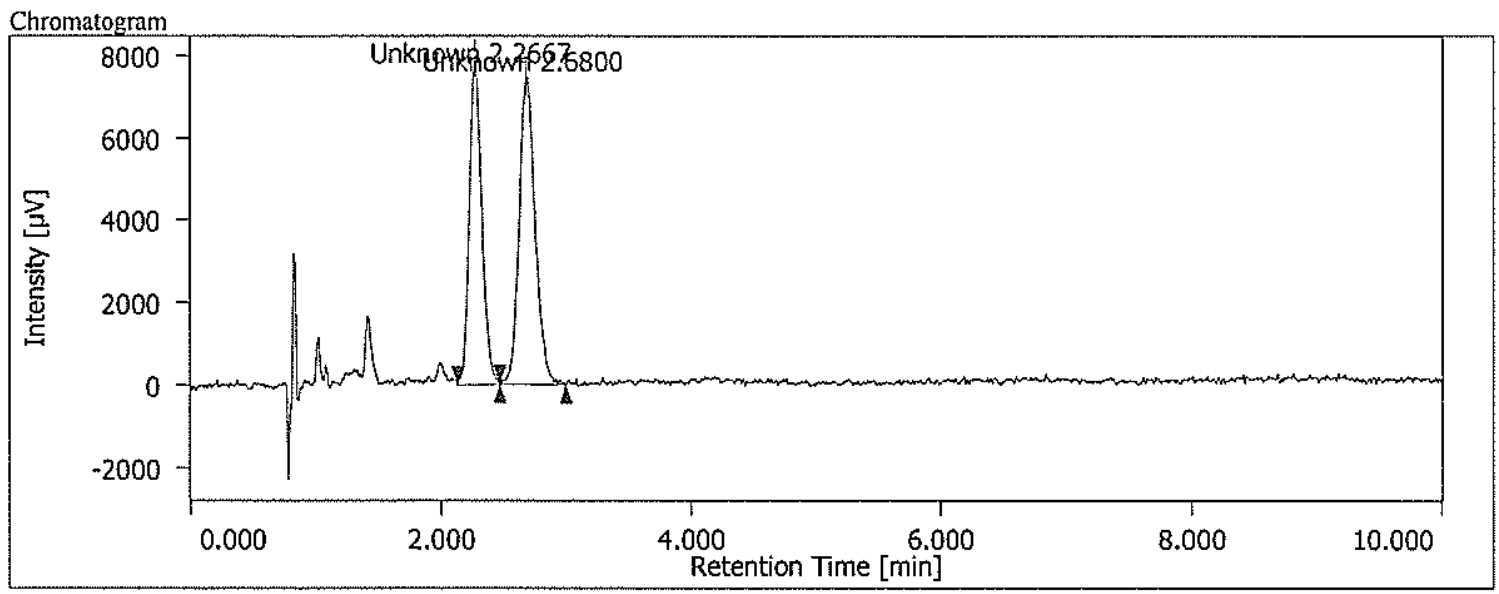

Peak Information

\begin{tabular}{|l|l|l|l|}
\hline$\#$ & $\mathrm{tR}$ [min] & Area [ $\mu \mathrm{V} \cdot \mathrm{sec}]$ & Area\% \\
\hline & & \\
\hline
\end{tabular}

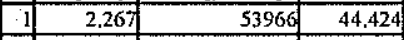

\begin{tabular}{ll|l|}
2.680 & 67514 & 55.576 \\
\hline
\end{tabular}

Figure S5. Enantiomeric mixture of 3k.

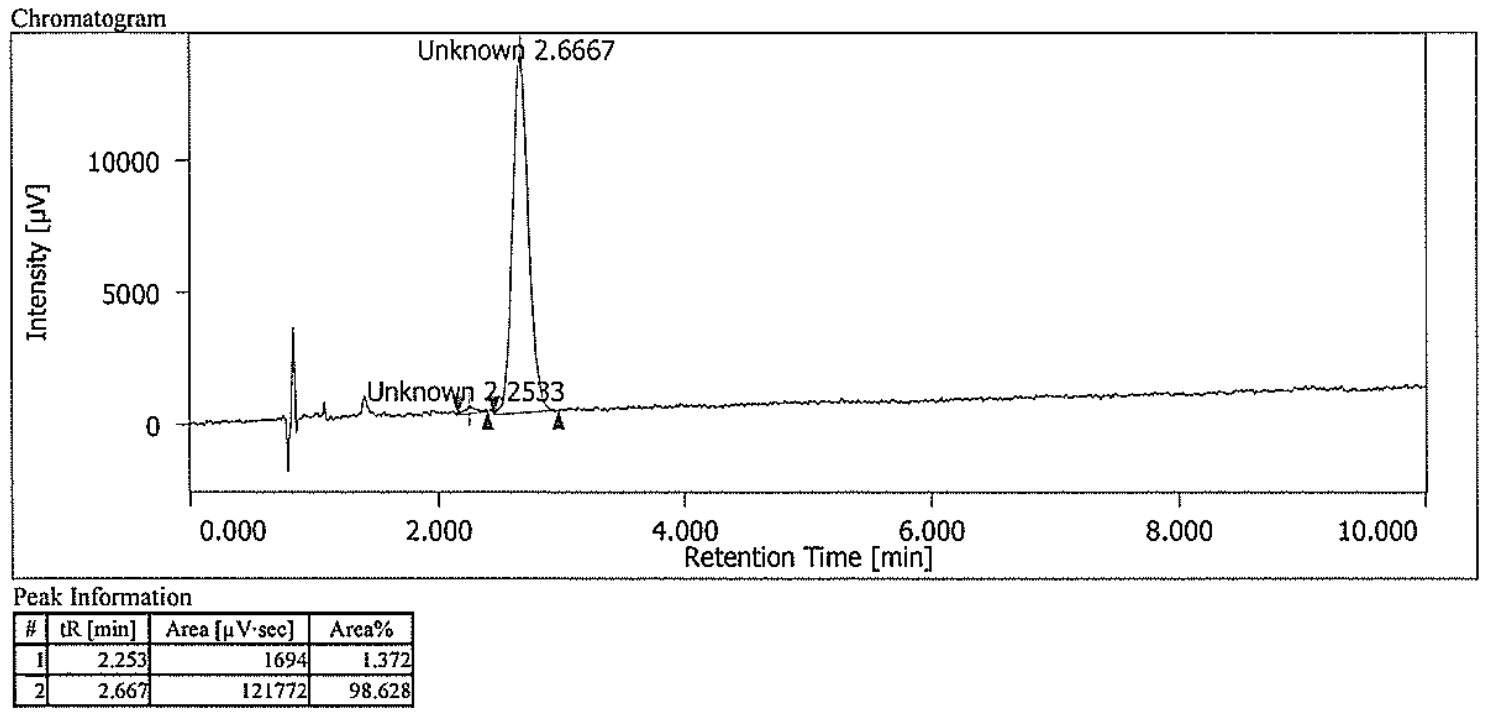

Figure S6. $(S)-3 \mathbf{k}$.<smiles>CCOC(=O)C(Cc1ccccc1)NC(=O)OCc1ccccc1</smiles>

\section{(S)-tert-butyl $N$-Boc-phenylalaninate (3I).}

The general procedure was followed with $\mathrm{NiBr}_{2}$ (diglyme) $(17.7 \mathrm{mg}, 0.0560 \mathrm{mmol}, 0.0700$ equiv), 4-4'-di-tert-butyl-2,2'-bipyridine ( $15.0 \mathrm{mg}, 0.0560 \mathrm{mmol}, 0.0700$ equiv), 1-(tert-butyl) 3(1,3-dioxoisoindolin-2-yl)-2-((tert-butoxycarbonyl)amino)malonate $(521 \mathrm{mg}, 1.20 \mathrm{mmol}, 1.50$ equiv), and iodobenzene ( $89.5 \mu \mathrm{L}, 0.800 \mathrm{mmol}, 1.00$ equiv). After $11 \mathrm{~h}$, the reaction mixture was filtered through a silica plug with diethyl ether, concentrated under reduced pressure, and 
purified by flash chromatography (95:5 hexane/acetone, $\left.\mathrm{R}_{\mathrm{f}}=0.24\right)$ to afford a light yellow oil $(192 \mathrm{mg}, 75 \%)$. Analytical data matched those in the literature. ${ }^{21}$

$[\alpha]_{\mathrm{D}}{ }^{23} 15.2\left(\mathrm{c}=1.05, \mathrm{CHCl}_{3}\right)$, which corresponds to $48-52 \%$ ee depending upon which literature value is used ( $32^{21}$ to $29.5^{22}$ have been reported). Chiral-phase SFC analysis demonstrates that the material is $97 \%$ ee (vide infra).

${ }^{1} \mathrm{H}$ NMR (400 MHz; $\mathrm{CDCl}_{3}$ ): $\delta 7.27$ (q, $\left.J=9.5 \mathrm{~Hz}, 4 \mathrm{H}\right), 7.17$ (d, $\left.J=6.8 \mathrm{~Hz}, 2 \mathrm{H}\right), 4.99$ (d, $J=7.1$ $\mathrm{Hz}, 1 \mathrm{H}), 4.45$ (q, $J=6.5 \mathrm{~Hz}, 1 \mathrm{H}), 3.05$ (d, $J=5.8 \mathrm{~Hz}, 2 \mathrm{H}), 1.41$ (d, $J=9.4 \mathrm{~Hz}, 18 \mathrm{H})$.

${ }^{13} \mathrm{C} \mathrm{NMR}\left(126 \mathrm{MHz}, \mathrm{CDCl}_{3}\right): \delta 171.1,155.2,136.5,129.6,128.4,126.9,82.1,79.7,55.0,38.66$, 28.4, 28.1 .

LRMS (ESI+) $m / z: 322.2\left[\mathrm{M}+\mathrm{H}^{+}\right], 344.2\left[\mathrm{M}+\mathrm{Na}^{+}\right]$.

SFC analysis (Daicel Chiralpak IC $(0.46 \mathrm{~cm} \mathrm{ID} \times 25 \mathrm{~cm} \mathrm{~L}), 10 \%$ modifier (4:1 hexanes/EtOH) in $\left.\mathrm{CO}_{2}, 4 \mathrm{~mL} / \mathrm{min}, \lambda=220 \mathrm{~nm}, 35^{\circ} \mathrm{C}, 12.0 \mathrm{MPa}\right)$ : $\mathrm{tR}[\mathrm{min}]=2.9(1.3 \%) 3.2(98.7 \%), 97 \%$ ee.

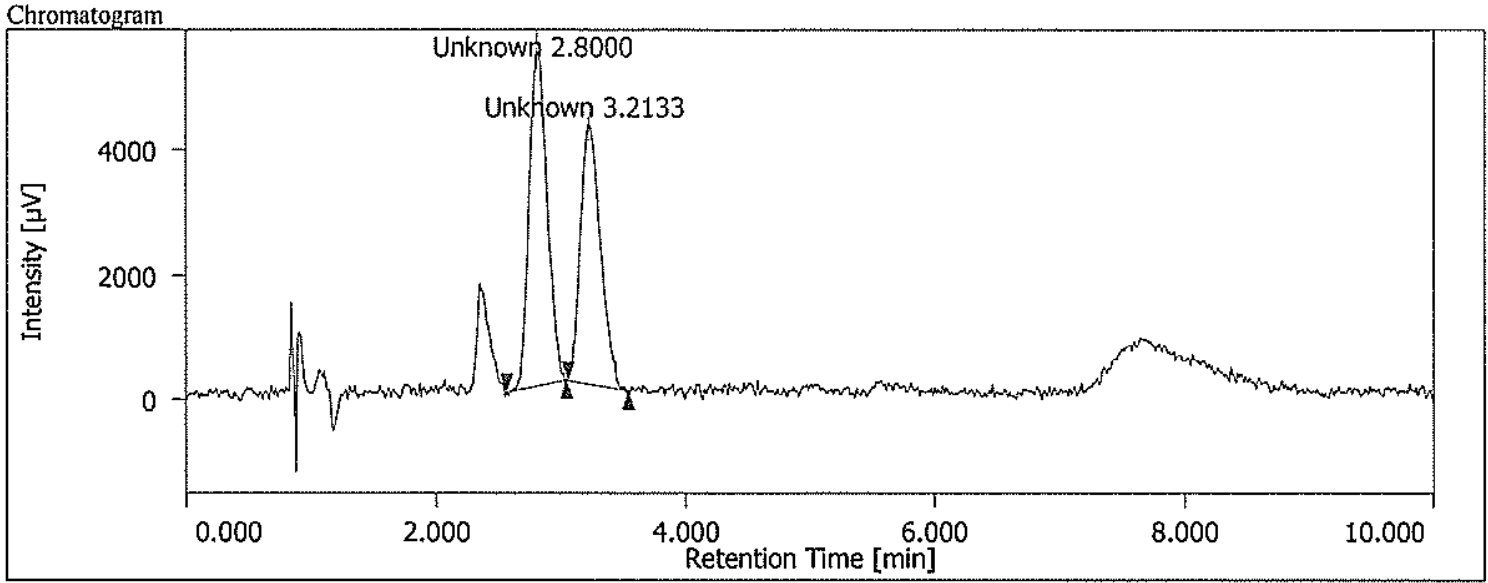

Peak Information
\begin{tabular}{|r|r|r|r|}
\hline \# & $\mathrm{tR}[\mathrm{min}]$ & Area [ $\mu \mathrm{V} \cdot \mathrm{sec}]$ & Area\% \\
\hline 1 & 2.800 & 54276 & 53.709 \\
\hline 2 & 3.213 & 46779 & 46.291 \\
\hline
\end{tabular}

Figure S7. Enantiomeric mixture of $\mathbf{3 1}$.

\footnotetext{
${ }^{21}$ Konda, Y.; Takahashi, Y.; Arima, S.; Sato, N. Takeda, K.; Dobashi, K.; Baba, M.; Harigaya, Y. Tetrahedron 2001, 57, 4311-4321.

${ }^{22}$ Strazzolini, P.; Melloni, T.; Giumanini, A. G. Tetrahedron 2001, 57, 9033.
} 


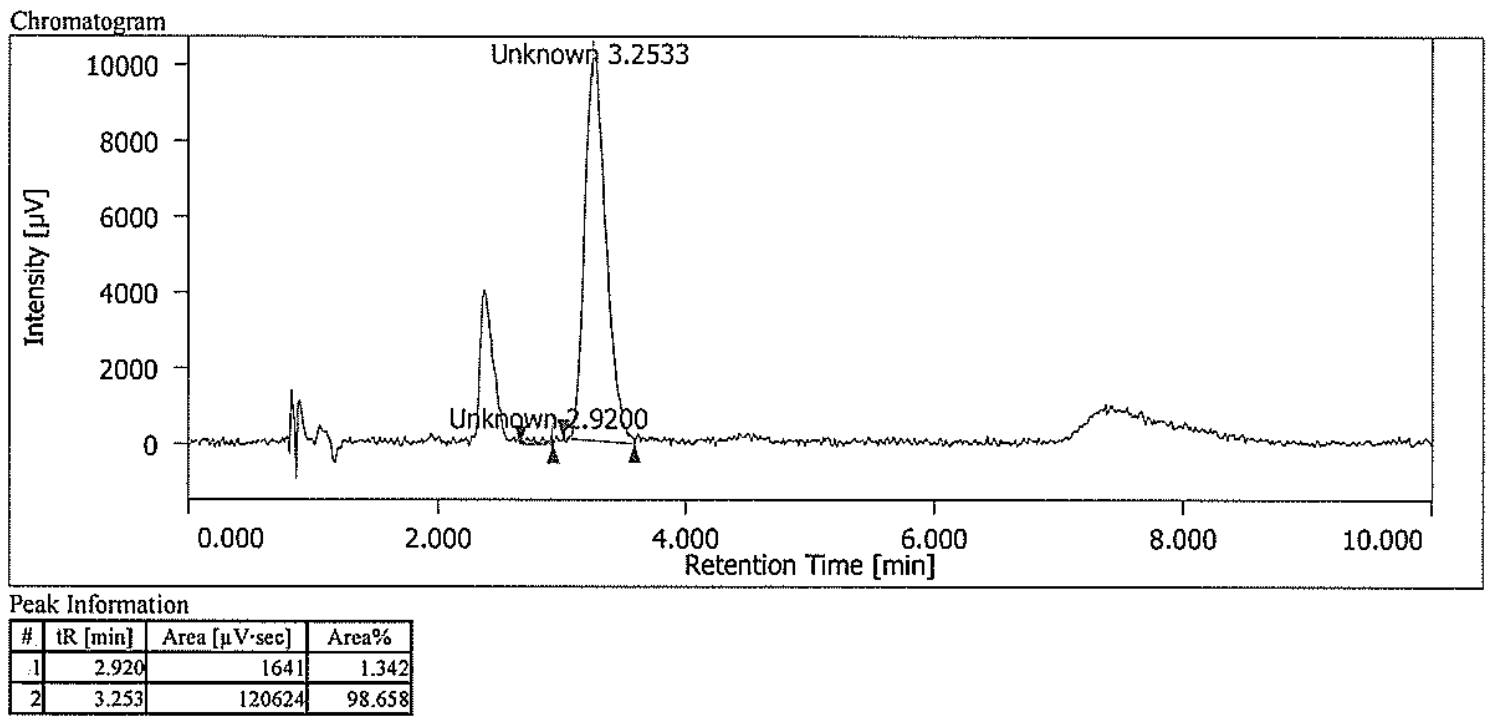

Figure S8. $(S)-31$.

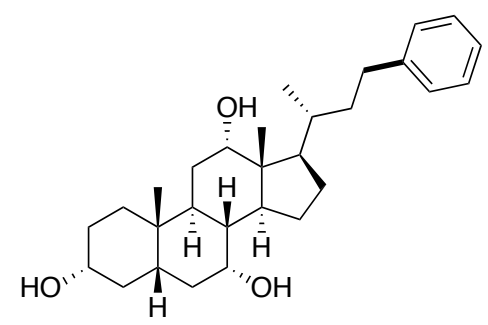

$(3 R, 5 S, 7 R, 8 R, 9 S, 10 S, 12 S, 13 R, 14 S, 17 R)-10,13-d i m e t h y l-17-((R)-4-p h e n y l b u t a n-2-$ yl)hexadecahydro- $1 H$-cyclopenta[ $[a]$ phenanthrene-3,7,12-triol $(3 \mathrm{~m})$.

The general procedure was followed with $\mathrm{NiBr}_{2}$ (diglyme) $(8.86 \mathrm{mg}, 0.0282 \mathrm{mmol}, 0.0700$ equiv), 4-4'-di-tert-butyl-2,2'-bipyridine (7.52 mg, $0.0282 \mathrm{mmol}, 0.0700$ equiv), 1,3dioxoisoindolin-2-yl $\quad(R)-4-((3 R, 5 S, 7 R, 8 R, 9 S, 10 S, 12 S, 13 R, 14 S, 17 R)-3,7,12$-trihydroxy-10,13dimethylhexadecahydro-1H-cyclopenta $[a]$ phenanthren-17-yl)pentanoate $(221 \mathrm{mg}, 0.600 \mathrm{mmol}$, 1.33 equiv), iodobenzene (91.0 mg, $0.450 \mathrm{mmol}, 1.00$ equiv), $\mathrm{Zn}$ (52.3 mg, $0.800 \mathrm{mmol}, 2.00$ equiv), and DMA $(0.500 \mathrm{~mL})$. After $5 \mathrm{~h}$, the reaction mixture was filtered through a silica plug, the plug was washed with diethyl ether, and the filtrate was concentrated under reduced pressure. The residue was purified by flash chromatography $\left(100 \%\right.$ EtOAc, $\left.\mathrm{R}_{\mathrm{f}}=0.44\right)$ to afford a colorless solid $(221 \mathrm{mg})$ that contained $N, N$-diisopropylurea $(\sim 10 \mathrm{wt} \%)$ and phthalimide $(\sim 4 \mathrm{wt} \%){ }^{23}$ Because we had difficulty separating these impurities from $\mathbf{3 m}$, we analyzed the product mixture by ${ }^{1} \mathrm{H}$ NMR with an internal standard (trimethoxybenzene) to determine the actual yield of product: (191 mg, 97\%). Peaks reported are for 3m only.

${ }^{1} \mathrm{H}$ NMR (400 MHz; $\mathrm{CDCl}_{3}$ ): $\delta$ 7.29-7.25 (m, 3H), 7.17 (d, J=6.0 Hz, 2H), $4.01(\mathrm{~s}, 1 \mathrm{H}), 3.85$ (s,

${ }^{23}$ The identity of these species was confirmed by GC analysis vs. authentic samples, GC-MS, and comparison of ${ }^{1} \mathrm{H}$ NMR chemical shifts. 
$1 \mathrm{H}), 3.45(\mathrm{ddd}, J=9.7,6.9,5.2 \mathrm{~Hz}, 1 \mathrm{H}), 2.72-2.68(\mathrm{~m}, 1 \mathrm{H}), 2.51-2.45(\mathrm{~m}, 1 \mathrm{H}), 2.25-2.16(\mathrm{~m}$, $1 \mathrm{H}), 1.99-1.86(\mathrm{~m}, 2 \mathrm{H}), 1.84-1.58(\mathrm{~m}, 8 \mathrm{H}), 1.57-1.48(\mathrm{~m}, 3 \mathrm{H}), 1.46-1.34(\mathrm{~m}, 3 \mathrm{H}), 1.29-1.23(\mathrm{~m}$, $1 \mathrm{H}), 1.02-0.94(\mathrm{~m}, 1 \mathrm{H}), 0.89-0.86(\mathrm{~m}, 3 \mathrm{H}), 0.68(\mathrm{~s}, 2 \mathrm{H})$.

${ }^{13} \mathrm{C}$ NMR (126 MHz, $\left.\mathrm{CDCl}_{3}\right): \delta 143.4,134.9,128.5$ 128.4, 125.7, 73.2, 72.1, 68.6, 47.42, 46.7, $42.0,41.6,39.8,39.7,38.1,35.6,35.4,34.9,34.8,32.8,30.7,28.4,27.7,26.7,23.4,22.7,17.9$, 12.7 .

$\operatorname{mp} 88-94{ }^{\circ} \mathrm{C}$

IR $\left(\mathrm{cm}^{-1}\right): 3352,2932,2862,2338,1790,1744,1640,1555,1450,1369,1234,1165,1076,1042$, 1018, 980, 748, 698 .

LRMS (ESI+) $m / z: 463.3\left[\mathrm{M}+\mathrm{Na}^{+}\right]$.

\section{Coupling of NHP ester with an acid chloride.}

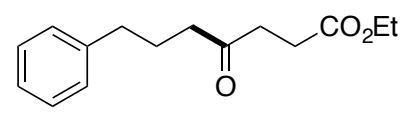

\section{ethyl 4-oxo-7-phenylheptanoate [108972-13-0] (5).}

The reaction was set up in a nitrogen filled glove box. To an oven-dried 1-dram vial, a Tefloncoated stir-bar was added. The catalyst was generated by pre-stirring $\mathrm{NiBr}_{2}$ (diglyme) (17.7 mg $0.0560 \mathrm{mmol}, 0.0700$ equiv) and 4-4'-di-tert-butyl-2,2'-bipyridine (15.0 mg, $0.0560 \mathrm{mmol}$, 0.0700 equiv) in DMA (1.00 mL) for $\sim 10 \mathrm{~min}$. Next, 1,3-dioxoisoindolin-2-yl 4-phenylbutanoate (248 mg, $0.800 \mathrm{mmol}, 1.00$ equiv), ethyl succinyl chloride (362 $\mu \mathrm{L}, 2.40 \mathrm{mmol}, 3.00$ equiv), dodecane as an internal standard $(10.0 \mu \mathrm{L})$, and zinc flake (105 mg, $1.60 \mathrm{mmol}, 2.00$ equiv) were consecutively added. The vial was capped with a screw cap fitted with a PTFE-faced silicone septum, removed from the glove box, and heated in a reaction block set to $28{ }^{\circ} \mathrm{C}$ on the benchtop with stirring at $1195 \mathrm{rpm}$. After $1 \mathrm{~h}$, the reaction mixture was worked up and purified by flash chromatography $\left(10 \%\right.$ EtOAc in hexanes, $\left.\mathrm{R}_{\mathrm{f}}=0.21\right)$ to afford a yellow oil (167 $\mathrm{mg}, 84 \%$ yield). Analytical data matched those in the literature. ${ }^{24}$

${ }^{1} \mathrm{H}$ NMR (400 MHz; $\left.\mathrm{CDCl}_{3}\right): \delta 7.28(\mathrm{t}, J=7.4 \mathrm{~Hz}, 2 \mathrm{H}), 7.18(\mathrm{t}, J=8.4 \mathrm{~Hz}, 3 \mathrm{H}), 4.12(\mathrm{q}, J=7.1$ $\mathrm{Hz}, 2 \mathrm{H}), 2.62(\mathrm{ddt}, J=32.2,15.9,7.6 \mathrm{~Hz}, 6 \mathrm{H}), 2.46(\mathrm{t}, J=7.3 \mathrm{~Hz}, 2 \mathrm{H}), 1.93$ (quintet, $J=7.4 \mathrm{~Hz}$, $2 \mathrm{H}), 1.25(\mathrm{t}, J=7.0 \mathrm{~Hz}, 3 \mathrm{H})$.

${ }^{13} \mathrm{C} \mathrm{NMR}\left(126 \mathrm{MHz}, \mathrm{CDCl}_{3}\right): \delta 208.7,172.8,141.6,128.5,128.4,126.0,60.6,41.9,37.2,35.1$, 28.0, 25.3, 14.2.

LRMS (ESI+) $m / z: 249.6\left[\mathrm{M}+\mathrm{H}^{+}\right], 271.5\left[\mathrm{M}+\mathrm{Na}^{+}\right]$.

${ }^{24}$ Debien, L.; Quiclet-Sire, B.; Zard, S. Z. Org. Lett. 2011, 13, 5676-5679. 


\section{Synthesis and reactions of (dtbbpy) $\mathrm{Ni}^{\mathrm{II}}(2$-tolyl)I (6)}

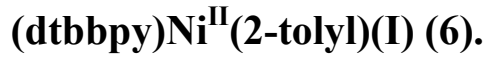

A solution of (dtbbpy)Ni(cod) was generated by stirring $138 \mathrm{mg}$ of $\mathrm{Ni}(\mathrm{cod})_{2}(0.5 \mathrm{mmol})$ and 134 $\mathrm{mg}$ of 4,4'-di-tert-butyl-2,2'-bipyridine $(0.5 \mathrm{mmol})$ in $5 \mathrm{~mL}$ of $\mathrm{THF}$ overnight at room temperature in an argon-filled glovebox. Upon dissolution of the solids, the reaction mixture became dark purple. To this purple mixture was added $0.076 \mathrm{~mL}$ of 2 -iodotoluene $(131 \mathrm{mg}, 0.6$ $\mathrm{mmol}$ ) and the color rapidly changed to red, indicating formation of $\mathbf{6}$. The solution was stirred for an additional $2 \mathrm{~h}$ before the solvent was removed under vacuum. The solid was triturated with dry, degassed pentane three times to remove residual cyclooctadiene and 2-iodotoluene and then dried under vacuum overnight to provide $230 \mathrm{mg}$ of 6 ( $85 \%$ yield). The ${ }^{1} \mathrm{H}$ and ${ }^{13} \mathrm{C} \mathrm{NMR}$ spectra are consistent with $\left[(\mathrm{dtbbpy}) \mathrm{Ni}(2\right.$-tolyl $\left.)\left(\mathrm{O}=\mathrm{C}\left(\mathrm{CD}_{3}\right)_{2}\right)\right] \mathrm{I}$ being the predominant species in solution, most notably the bound acetone- $d_{6}$ in the ${ }^{13} \mathrm{C}$ NMR gives rise to characteristic peaks at 209.9 (s) and 54.6 (apparent q, expected septet for $-\mathrm{CD}_{3}$ ). The propensity of $\mathrm{Ni}(\mathrm{II})$ halide complexes to exist as solvated cations is well documented. ${ }^{25}$

${ }^{1} \mathrm{H}$ NMR (500 MHz; acetone- $\left.d_{6}\right): \delta 9.56(\mathrm{~d}, J=2.9 \mathrm{~Hz}, 1 \mathrm{H}), 8.40(\mathrm{~d}, J=16.4 \mathrm{~Hz}, 2 \mathrm{H}), 7.65(\mathrm{~s}$, $1 \mathrm{H}), 7.57(\mathrm{~d}, J=6.9 \mathrm{~Hz}, 1 \mathrm{H}), 7.38(\mathrm{~s}, 1 \mathrm{H}), 6.84(\mathrm{~d}, J=2.8 \mathrm{~Hz}, 1 \mathrm{H}), 6.76(\mathrm{~d}, J=5.7 \mathrm{~Hz}, 1 \mathrm{H})$, 6.70-6.69 (m, 1H), $6.63(\mathrm{~d}, J=6.8 \mathrm{~Hz}, 1 \mathrm{H}), 2.95(\mathrm{~s}, 3 \mathrm{H}), 1.43(\mathrm{~s}, 9 \mathrm{H}), 1.37(\mathrm{~s}, 9 \mathrm{H})$.

${ }^{13} \mathrm{C}$ NMR $\left(126 \mathrm{MHz}\right.$, acetone- $\left.d_{6}\right): \delta 209.94,164.42,164.01,156.64,154.20,154.04,149.82$, $148.97,143.42,138.49,127.80,124.37,124.28,123.63,122.62,119.66,119.25,69.15,54.64$ (q, $J=19.1 \mathrm{~Hz}), 36.18$ (2C), 30.37, 27.20.

Anal. Cald for $\mathrm{C}_{25} \mathrm{H}_{31} \mathrm{~N}_{2} \mathrm{NiI}$ : $55.08 \% \mathrm{C}, 5.73 \% \mathrm{H}, 5.14 \% \mathrm{~N}$; found $53.75 \% \mathrm{C}, 5.69 \% \mathrm{H}, 4.80 \%$ N.

UV-Vis spectrum in DMF (Figure S9): $\varepsilon_{430}=2598 \mathrm{M}^{-1} \mathrm{~cm}^{-1}$.

25 (a) Osakada, K.; Yamamoto, T. Coord. Chem. Rev. 2000, 198, 379-399; (b) Ishiguro, S.-i.; Ozutsumi, K. Bull. Chem. Soc. Jpn. 1989, 62, 2392-2393. 


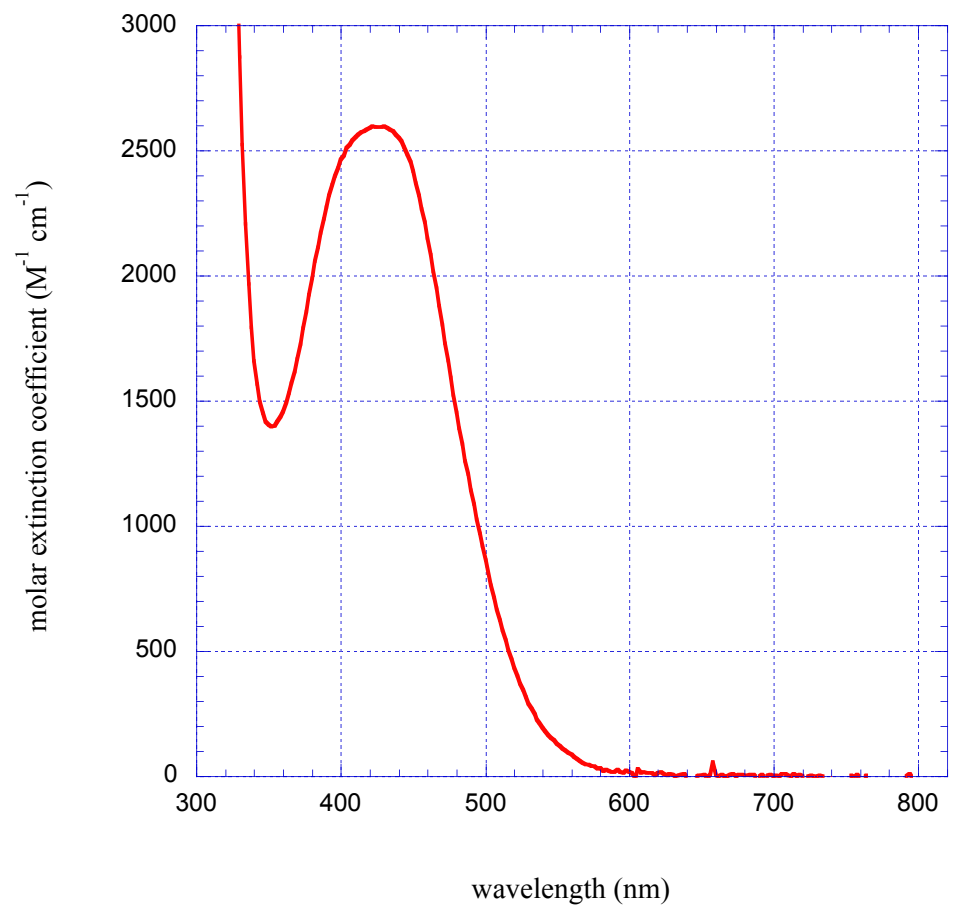

Figure S9. UV-vis of 6 recorded in DMF at rt.

\section{NMR analysis of reaction of $\mathbf{6}$ with $1 \mathrm{~b}$.}

A solution of $0.0125 \mathrm{mmol}(6.8 \mathrm{mg})$ (dtbbpy) $\mathrm{Ni}^{\mathrm{II}}(2$-tolyl)(I) (6) was generated in $0.75 \mathrm{~mL}$ of DMF- $d 7$. To this solution was added 1,3-dioxoisoindolin-2-yl 4-phenylbutanoate (1b) $(0.13$ $\mathrm{mmol}, 40 \mathrm{mg}$ ). During this time the color changed from red to orange indicating reaction. The resulting orange homogenous solution was then analyzed by ${ }^{1} \mathrm{H}$ NMR using paramagnetic parameters. Consumption of $\mathbf{6}$ was confirmed in the diamagnetic region (Figure S10). A paramagnetic species was present in the reaction mixture as indicated by broad peaks at 60,41 , and $19 \mathrm{ppm}$ (Figure S11). Examination of this material by EPR spectroscopy at both $\mathrm{rt}$ and $15 \mathrm{~K}$ found no signal, consistent with an integer-spin system.

UV-Vis (Figure S12): $\varepsilon_{440}=1931 \mathrm{M}^{-1} \mathrm{~cm}^{-1}$. 


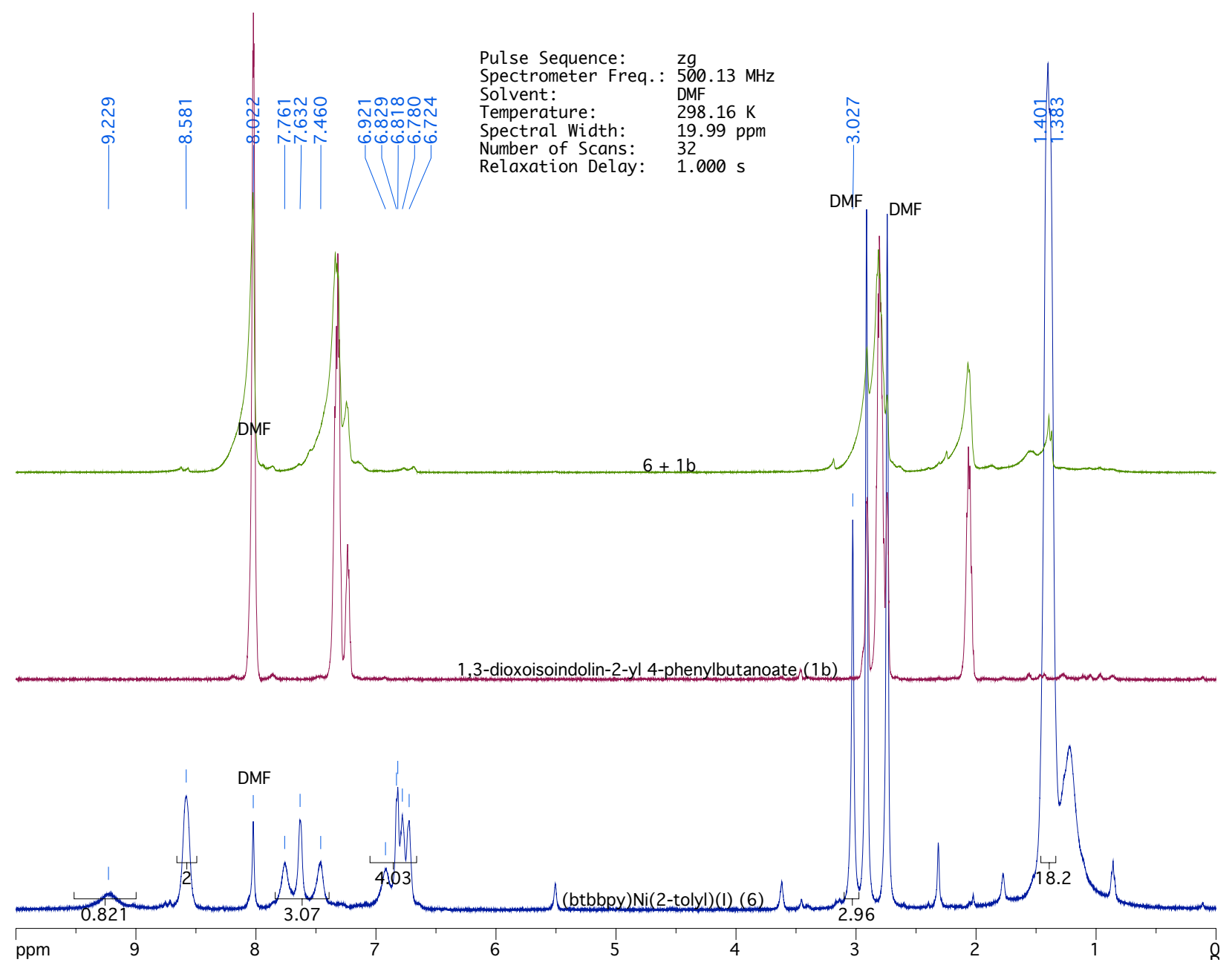

Figure S10. ${ }^{1} \mathrm{H}$ NMR spectra of $\mathbf{6}, \mathbf{1 b}$, and $\mathbf{6}+\mathbf{1 b}$ after stirring overnight in DMF- $d_{7}$ at rt. 


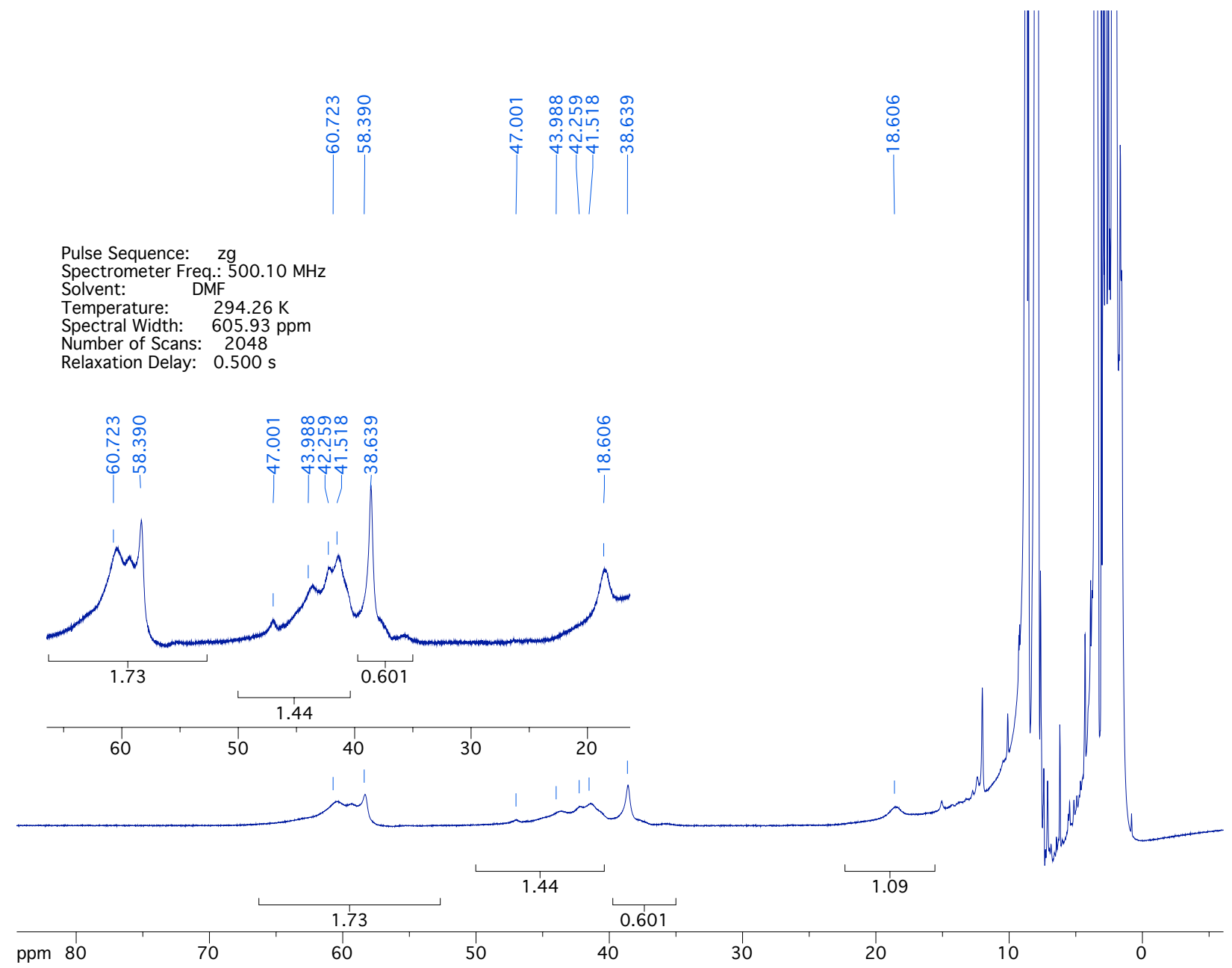

Figure S11. Paramagnetic ${ }^{1}$ H NMR spectrum of $\mathbf{6}+\mathbf{1 b}$ after stirring overnight in DMF- $d_{7}$ at rt. 


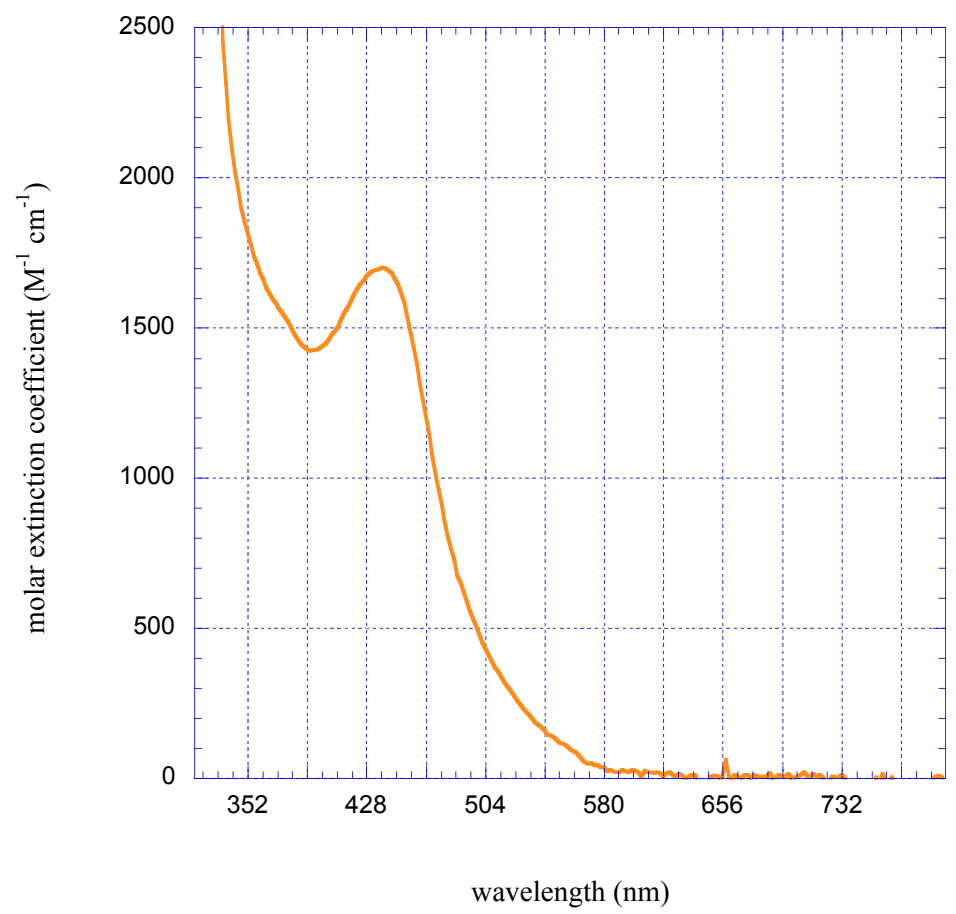

Figure S12. UV-vis spectrum of $\mathbf{6}+\mathbf{1 b}$ after stirring overnight in DMF- $d_{7}$ at rt.

\section{Reaction of (dtbbpy) Ni ${ }^{\text {II }}$ (2-tolyl)(I) (6) with 1 b.}

To a $1 \mathrm{~mL}$ oven-dried conical vial equipped with a PTFE-coated stir-vane was added (dtbbpy) $\mathrm{Ni}^{\mathrm{II}}$ (2-tolyl)(I) (6) (6.8 mg, $\left.0.0125 \mathrm{mmol}\right), 1,3$-dioxoisoindolin-2-yl 4-phenylbutanoate (1b) $(40 \mathrm{mg}, 0.13 \mathrm{mmol})$, dodecane $(10 \mu \mathrm{L}, 0.044 \mathrm{mmol})$, and $250 \mu \mathrm{L}$ of DMF. The vial was capped with a PTFE-faced silicone septum, removed from the glovebox, and set to stir at room temperature. GC analysis of acid-quenched aliquots of this reaction mixture showed product $\mathbf{3 h}$ ( $47 \%$ average yield over 3 runs, corrected GC yield). We could not account for all of the mass of 6 added to the reaction mixture - only trace toluene and no 2,2'-bitoluene were observed.

\section{Reaction of (dtbbpy)Ni ${ }^{\text {II }}$ (2-tolyl)(I) (6) with 1 b and $\mathrm{Zn}$.}

In a $1 \mathrm{~mL}$ oven-dried conical vial equipped with a PTFE-coated stir-vane was added (dtbbpy) $\mathrm{Ni}^{\mathrm{II}}$ (2-tolyl)(I) (6) (6.8 mg, $\left.0.0125 \mathrm{mmol}\right), 1,3$-dioxoisoindolin-2-yl 4-phenylbutanoate (1b) $(40 \mathrm{mg}, 0.13 \mathrm{mmol})$, zinc $(1.6 \mathrm{mg}, 0.025 \mathrm{mmol})$, dodecane $(10 \mu \mathrm{L}, 0.044 \mathrm{mmol})$, and 250 $\mu \mathrm{L}$ of DMF. The vial was capped with a PTFE-faced silicone septum, removed from the 
glovebox, and set to stir at room temperature. GC analysis of a acid-quenched $\left(\mathrm{NaHSO}_{4}\right.$ (aq) $)$ aliquots of this reaction mixture showed product $\mathbf{3 h}$ ( $98 \%$ average yield over 3 runs, corrected GC yield). At later reaction points, propylbenzene and 1,6-diphenylhexane started growing in, presumably due to the action of zinc and nickel in the absence of an aryl electrophile.

\section{Catalytic reaction of 2-iodotoluene with $1 \mathrm{~b}$ in DMF.}

Run as the general procedure on $0.4 \mathrm{mmol}$ scale with $\mathbf{1 b}$ and 2-iodotoluene in a 1:1 ratio (vide supra) except in dry DMF. Yield was calculated by GC vs. an internal standard (dodecane). After $4 \mathrm{~h}, \mathbf{1 b}$ had been completely consumed but 2-iodotoluene remained. In addition to a $46 \%$ yield of $\mathbf{3 h}$, a substantial amount of 1,6-diphenylhexane (the dimer derived from $\mathbf{1 b}$ ) was also present.

\section{Reaction of ethyl 3-iodobenzate with zinc.

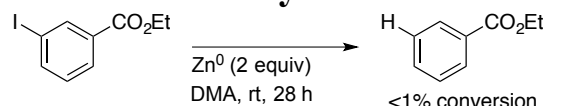

Ethyl 3-iodobenzoate was subjected to standard reaction conditions (vide supra) except that nickel and ligand were omitted from the reaction. The mixture was stirred at rt for 28 hours and monitored by GC analysis. Comparison of the chromatograms to chromatograms of authentic starting material and ethyl benzoate confirmed that $<1 \%$ of the aryl iodide was reduced in $28 \mathrm{~h}$. For comparison, catalytic reactions are complete in $12 \mathrm{~h}$ or less.

\section{NMR Spectra}



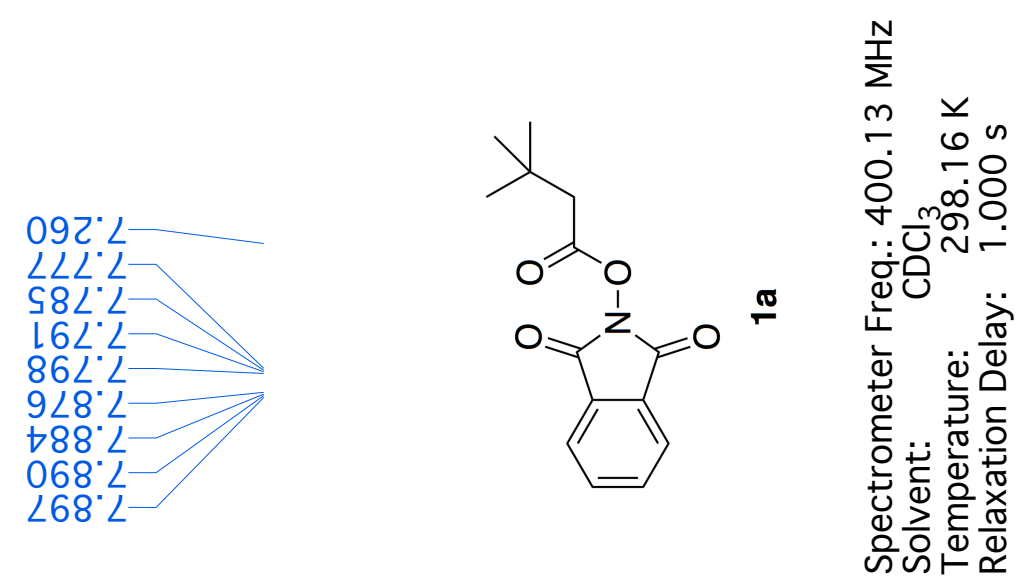


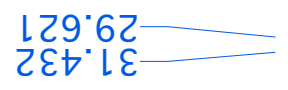

Oヤட゙ャヤ

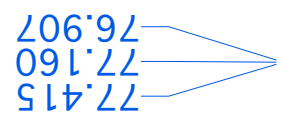

ยSO॰ヤ乙L吅62 L

$\varepsilon \varepsilon 8 " \triangleright \varepsilon\llcorner$

L 6 L"29 L

S66 $\angle 9 \mathrm{~L}$

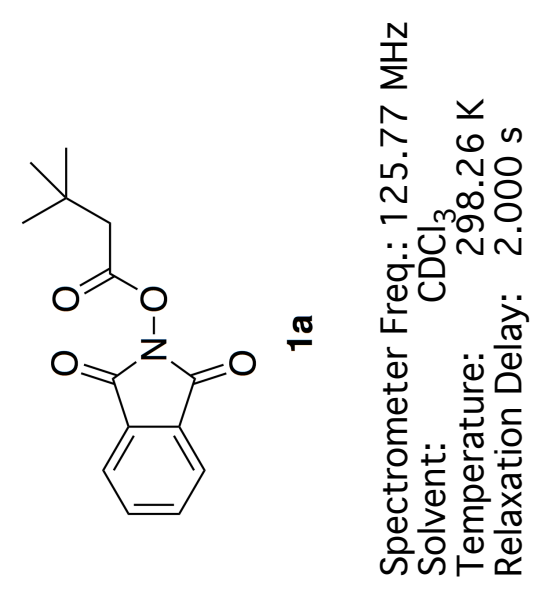


$5 \angle 0^{\circ} \mathrm{Z}$

$\angle \angle O^{\circ}$

$\varepsilon 80.2$

$20 \mathrm{~L}^{\circ} \mathrm{Z}$

$02 \mathrm{~L}$ "

$6 \varepsilon l \cdot 2$

$8 \mathrm{SL} 2$

$2 \mathrm{~L}$.

959.

$\rightarrow \angle 9^{\circ}$

269.

192

$08 L$

$66 L ' 2$
$022: \angle$

$6 \varepsilon 2^{\circ}$

962.

$\rightarrow\left\llcorner\varepsilon^{\circ}\right.$

$\neg \varepsilon \varepsilon^{\circ}$

$\rightarrow 8 L^{\circ}$

$26 L^{\circ} \mathrm{L}$

$86 L^{\circ}$

$508^{\circ}$

$588^{\circ} \mathrm{L}$

$\varepsilon 68^{\circ}$

$868^{\circ}$

$906^{\circ}$

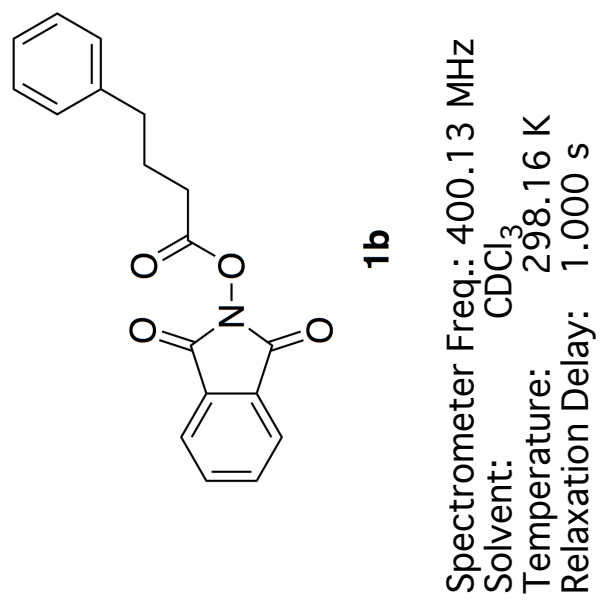

$\infty$

$\neg-\tilde{m}] \stackrel{\sim}{\sim}$

굴

$-\infty$ 
$02 l+t z$
$8 \angle \varepsilon=92$

$\angle 89^{\circ} 82$

$12 L .82$

†L L'6ZL

$968{ }^{\circ} \downarrow \varepsilon L$

0 L OH L $^{\circ}$

LLL'29L

195.69 L

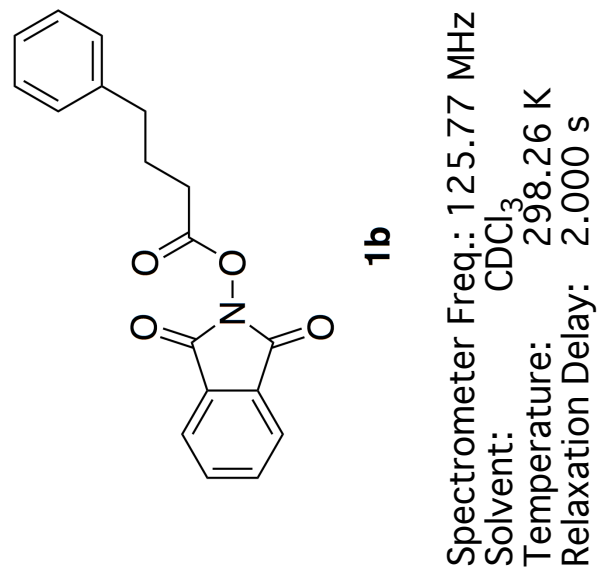

S36 


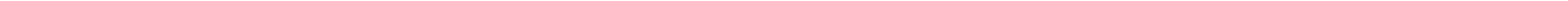


$6 \angle 0.92$

$0 \angle \mathcal{E}^{\circ} 0 \varepsilon$

L L $8^{\circ} 0 t$

S06.9L

$09 l^{\circ}<L$

8\&0"†乙L 99 l'62 L-

เ เ $\downarrow \varepsilon\llcorner$

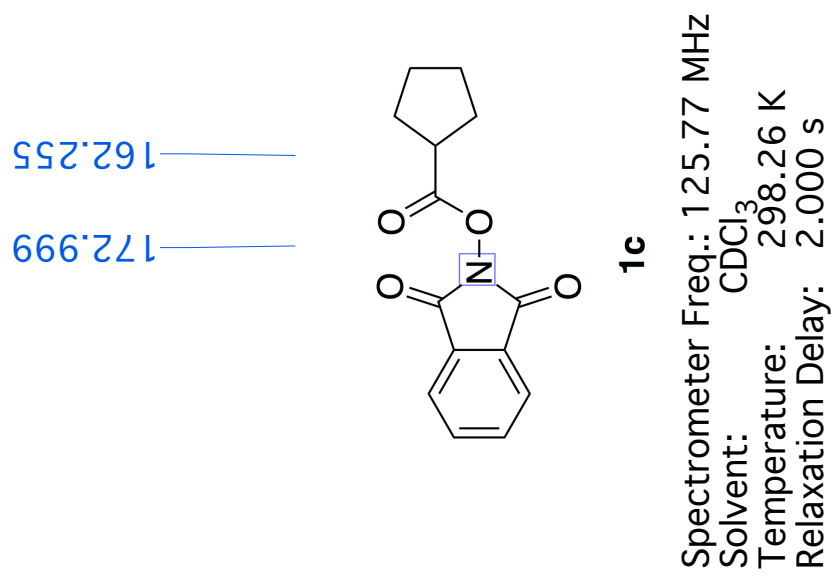




\section{$6 \angle 8^{\circ}$ \\ S06. \\ $626^{\circ} \mathrm{L}$ \\ $S \angle O^{\circ} \mathrm{Z}$ \\ LOL'Z \\ 906.2 \\ S $16^{\circ} \mathrm{C}$ \\ $1 \rightarrow 6^{\circ} 2$ \\ IS6.2 \\ $996^{\circ} 2$ \\ $9 \angle 6^{\circ} 2$ \\ S60 $\varepsilon$ \\ $02 L^{\circ} \varepsilon$ \\ $9 b l^{\circ} \varepsilon$}

$660^{\circ} \bullet$

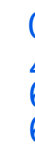

LOE:

6乙E.

$\neg \varepsilon \varepsilon^{\prime \prime} L$

$\downarrow \downarrow \varepsilon \mathcal{C}^{\circ}$

L $9 \varepsilon^{\circ} 2$

S $8 L$

E $6 L^{\circ}$

$66 L^{\circ}$

908.

9 l.

$698^{\circ}$

$6 \angle 8^{\circ} \mathrm{L}$

$\angle 88^{\circ} \mathrm{L}$

E68.'

$006^{\circ} \mathrm{L}$

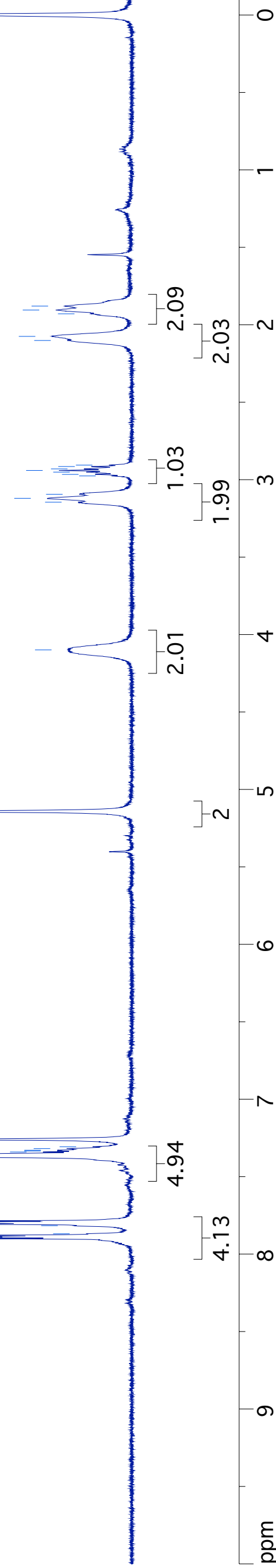




\section{$2 \angle L \angle Z$}

$187^{\circ} 8 \varepsilon$

$888^{\circ} \mathrm{t} t$

\section{$\angle 8 \varepsilon^{\circ} \angle 9$}

$\angle 06.92$

$091^{\circ}<2$

$9 l 1 . t 2$

$981^{\circ} 82$

$\rightarrow 20.621$

$1 \varepsilon 6^{\circ} \forall \varepsilon$

$\rightarrow S L^{\circ} 9 \varepsilon L$

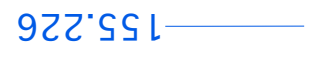

ع86. $19 \mathrm{~L}$

†09.0L
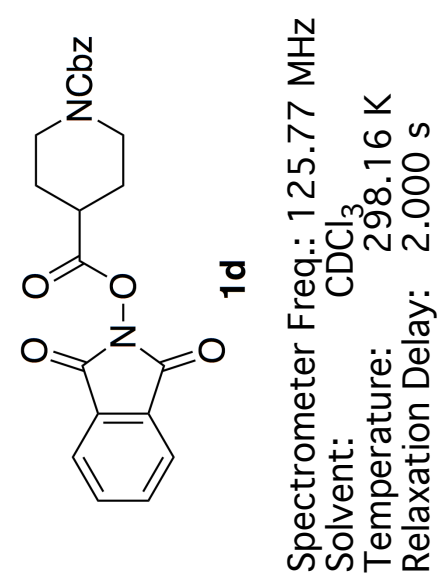
LIS. L 276 . 2S6. $\mathrm{L}$ $796^{\circ}$

$\angle \angle 6^{\circ}$ $\angle 86^{\circ}$ $6 \varepsilon 0^{\circ}$ $\triangle S 0^{\circ} \mathrm{Z}$ $1 \angle O^{\circ} \mathrm{C}$ $980^{\circ}$ E60'ح

L L L'C

$92 l^{\circ} \mathrm{C}$

29ع ट

$\downarrow \angle \varepsilon$

$\varepsilon 8 \varepsilon$

七Ot 2

$88 t^{\circ}$ $667^{\circ}$

009.

S $19^{\circ} \mathrm{t}$

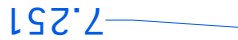
$\varepsilon S L^{\circ}$

8SL.

$\varepsilon 9 L$ $\angle 9 L$ 958. $198^{\circ}$

$998^{\circ}$

L $\angle 8^{\circ} \mathrm{L}$

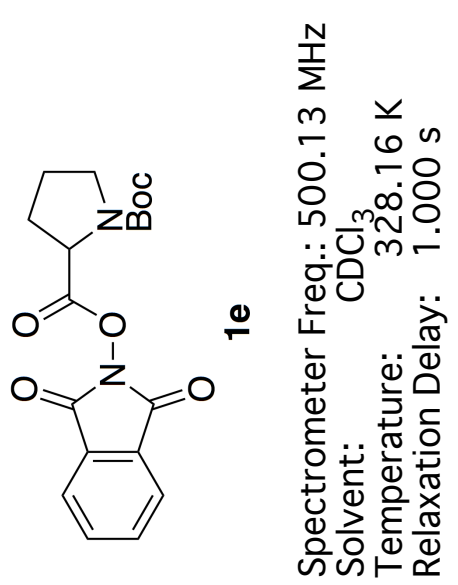


$\dashv\left\lfloor 9^{\circ} \varepsilon \mathcal{L}\right.$

59l.82

0†t:82

stc: 97

$\varepsilon \downarrow \mathcal{S}^{\prime} 9 t$

$9 \angle L \angle S$

$\angle 92^{\circ} \angle S$

S06:9L

091 L $L L$

L $\angle L '$ L8
ᄂ LO"†乙 L

S†6"8己 L

$8 S L t \varepsilon L$

$\varepsilon 68$ " $\downarrow \varepsilon l$

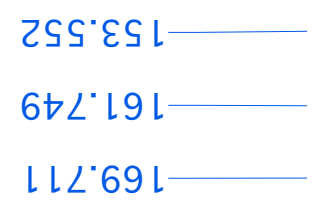



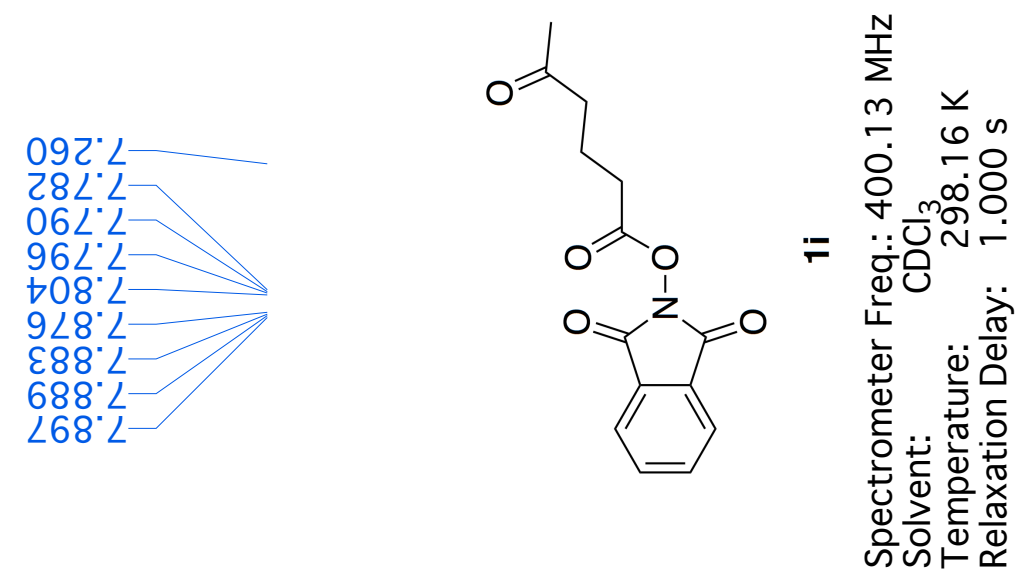
ع69"8 L-

ZLO०OE-
EOZ

ธย9"เฤ

906:9L

$09 L^{\circ} \angle L$

SLt $2 L$

乙Lし๋乙L

$\angle \angle 6^{\circ} 82 \mathrm{~L}$

$\angle \varepsilon 6^{\circ} \triangleright \varepsilon \mathrm{L}$

OSO"29L-

$88 \varepsilon^{\circ} 69$ L

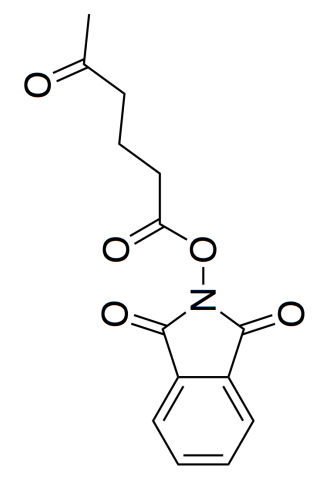

$679 * \angle 02$

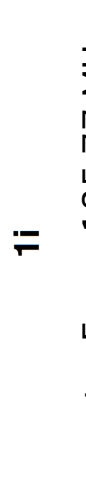

$\stackrel{N}{\Sigma}$

$\curvearrowright \underline{0}$

กิ

= $\because$ mన̃

:ㅇํㅇ

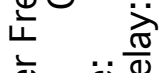

๑)

동. 둔

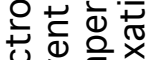

d릉

ज윰ำ

S44 


\section{$8 \nabla S^{\circ} L$}

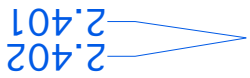

092 L $\varepsilon 08^{\circ} \mathrm{L}$

988'L

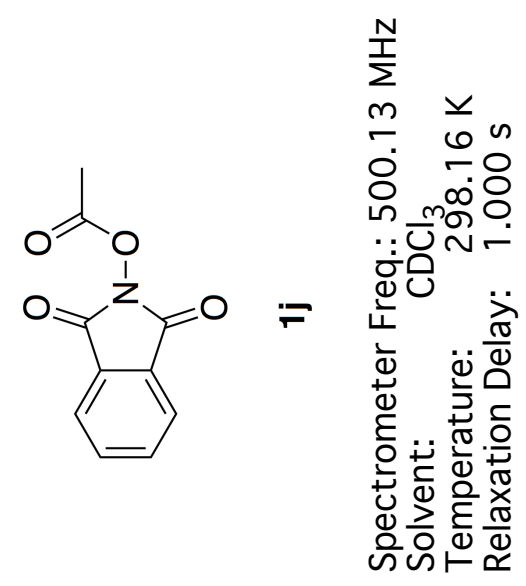



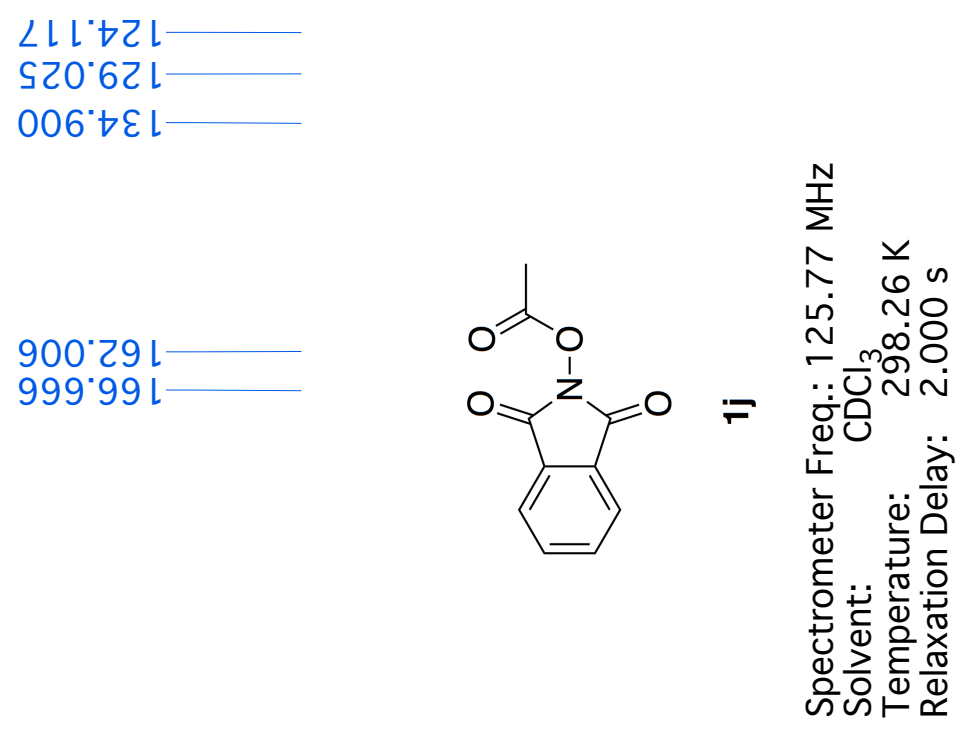
OOS $\angle 2$

880.82

$96 \varepsilon^{\circ} 82$

$68 L^{\prime} \varepsilon S$

906:9L

$09 \mathrm{~L}^{\circ} \angle 2$

$6 \angle 00^{\circ} 08$

SZL'Z8

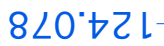

$6 \succ 6.82 \mathrm{~L}$

$968^{\circ} \downarrow \varepsilon L$

96t"SSL

†68. $19 \mathrm{~L}$

L $\angle 0^{\circ} 69 \mathrm{~L}$ $\angle 68^{\circ} 0 \angle \mathrm{L}$
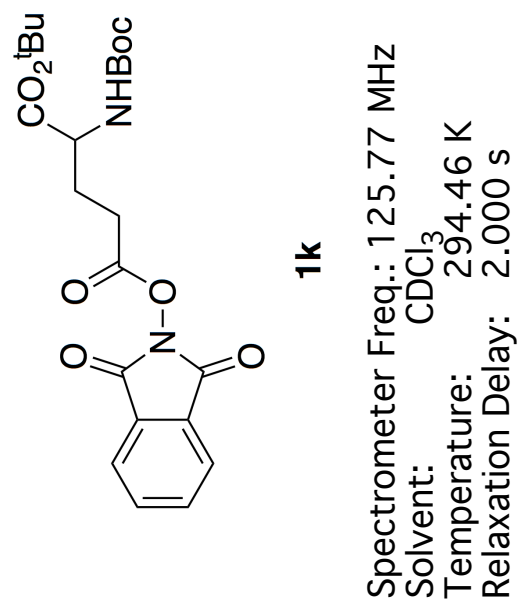

S48

8 
$\succ 9 \nabla^{\circ} \mathrm{L}$

$\varepsilon 8 t^{\circ}$

$6 \varepsilon \mathcal{C}^{\circ} \varepsilon$

OSZ

เOE. $\varepsilon$

$0\left\llcorner\varepsilon^{\prime} \varepsilon\right.$

2
7
9
6
1

$\forall \angle S^{\circ} \forall$

$9 \angle S " \nabla$

$6 \angle S^{\circ} t$

L8S"

$78 S^{\circ} \rightarrow$

885. $t$

$68 S^{\circ} t$

L6S" 7

E6t.

Els.
092:L$\varepsilon 8 L^{\circ} L$

L $6 L^{\circ}$

$96 L$

$\rightarrow 08^{\circ} 2$

$6 \angle 8^{\circ} \angle$

$\angle 88^{\circ} \mathrm{L}$

$268^{\circ} 2$

$006^{\circ} \mathrm{L}$
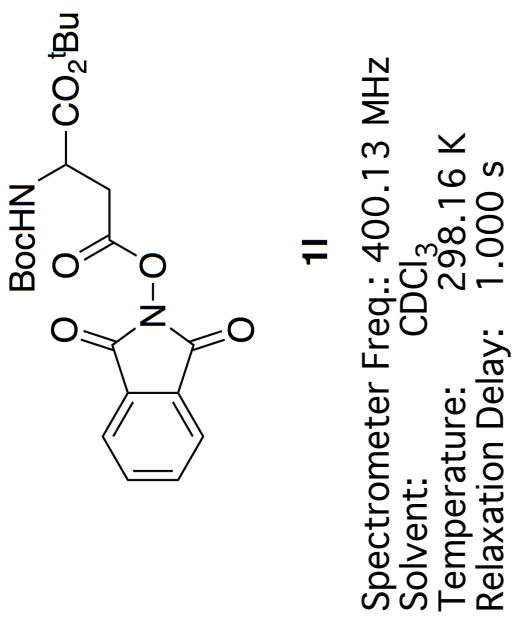

$\sqrt{-\infty}$

$\sqrt{\frac{6}{\sim}}$

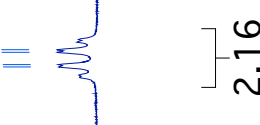

$=\left\{\begin{array}{l}\hat{\sigma} \\ 0\end{array}\right.$

$=$ =

$-\infty$

$\frac{\varepsilon}{2}$ 
$9 S 8^{\circ} \angle 2$

$86 \varepsilon^{\prime} 82$

$\varsigma 8 乙^{\circ} \downarrow \varepsilon$

†92.0s

906:9L

$S I t: L L$

$662^{\circ} 08$

$8 \sqcup \varepsilon^{\circ} \varepsilon 8$
80 レ゙ヤてい

ऽะ6.8Z L

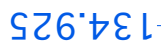

LItৎS L

LS9.19L

$8 S^{\circ} \angle 9 \mathrm{~L}$

七ट $89 \mathrm{~L}$
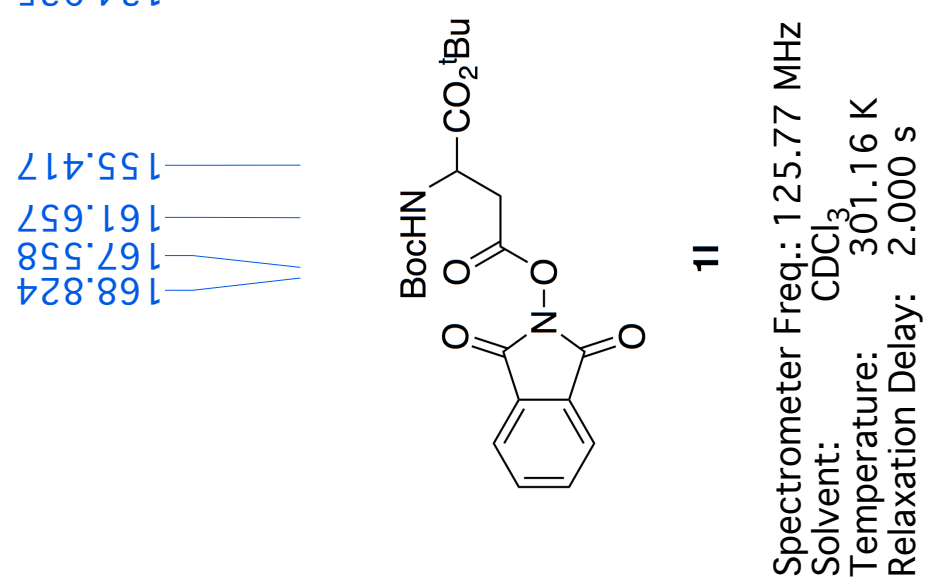


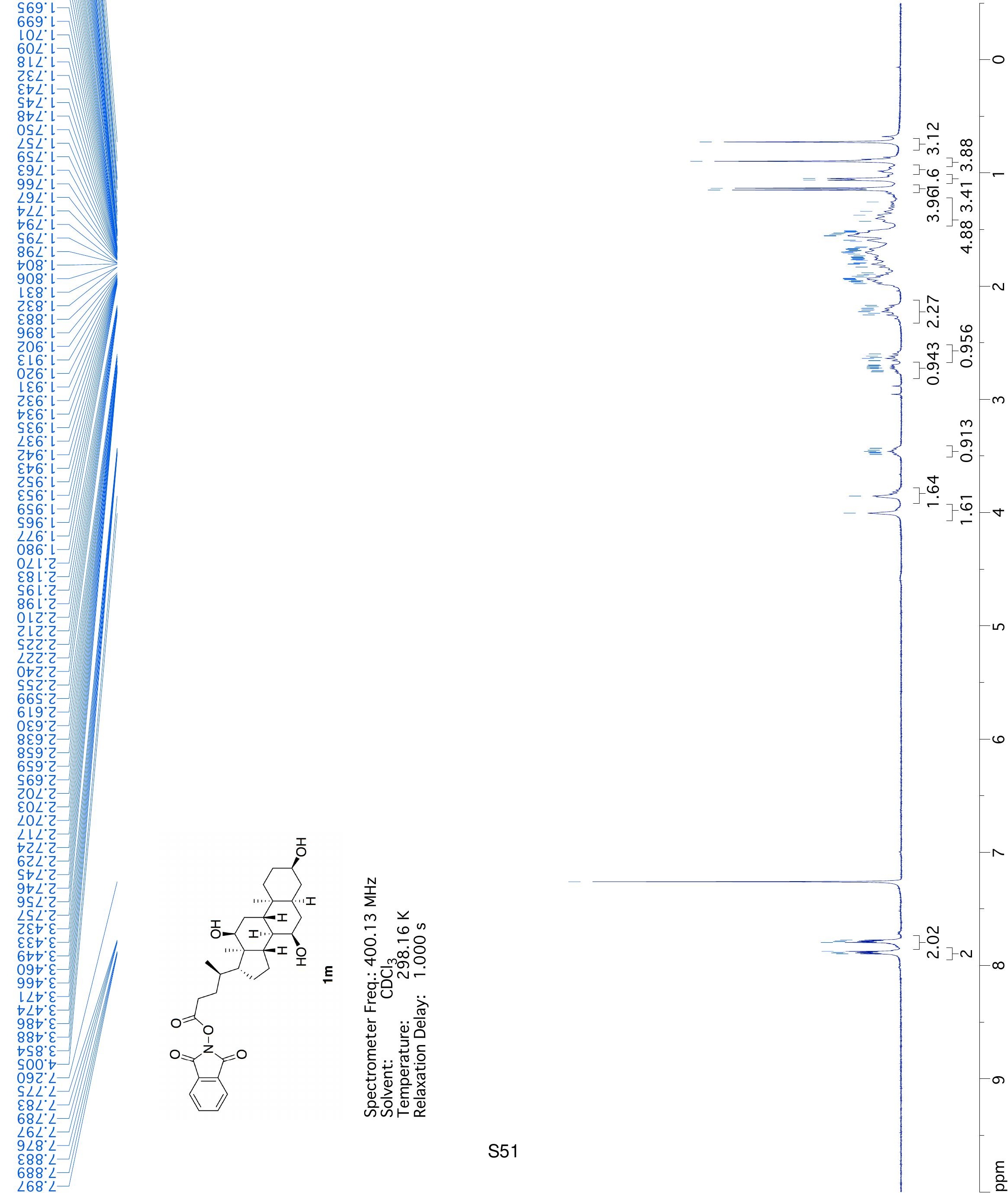




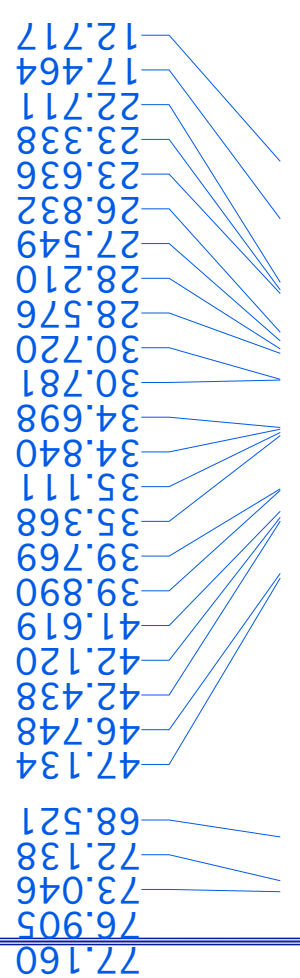

091.2L

$\varepsilon L \nabla^{\circ} L L$

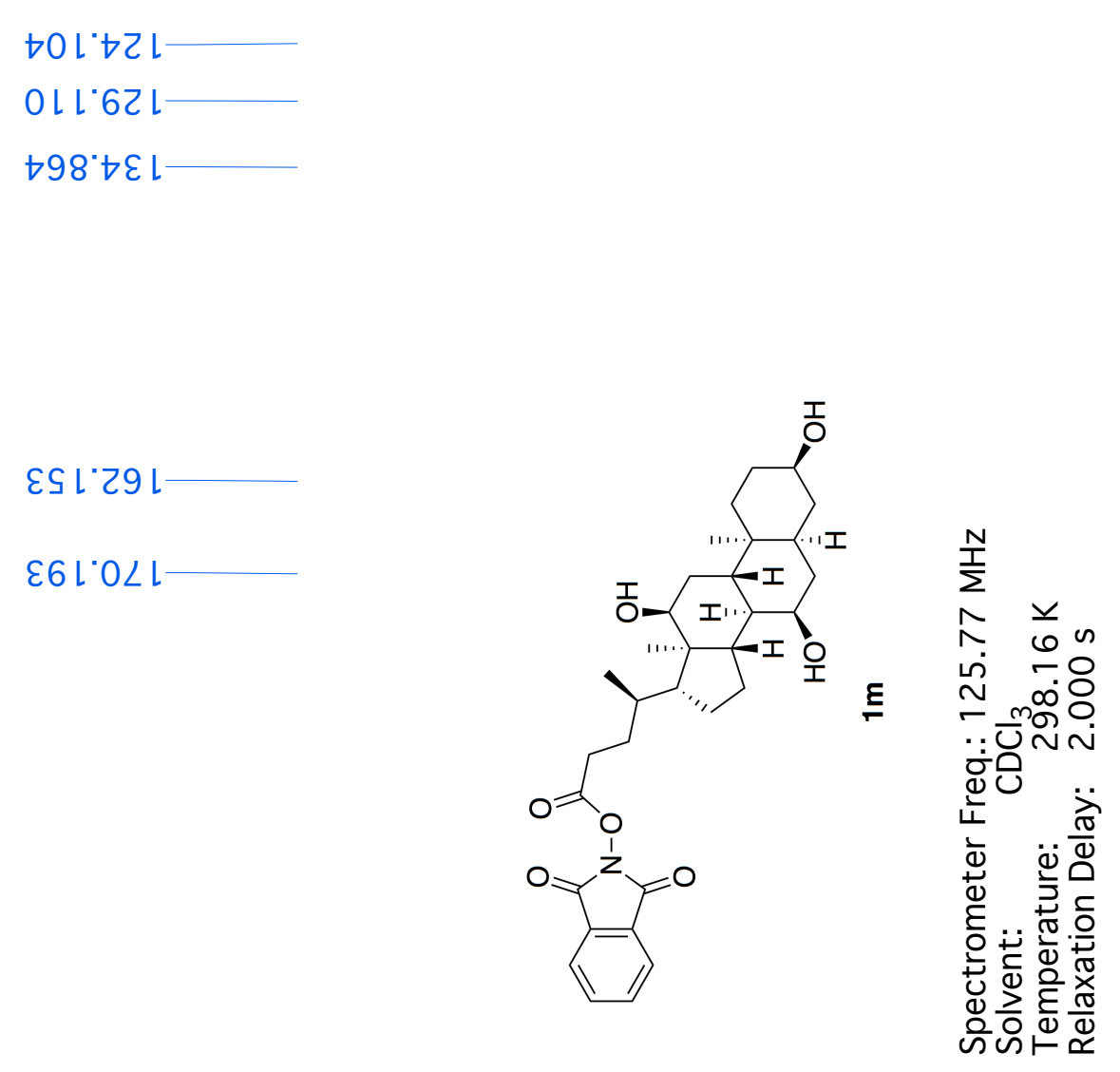




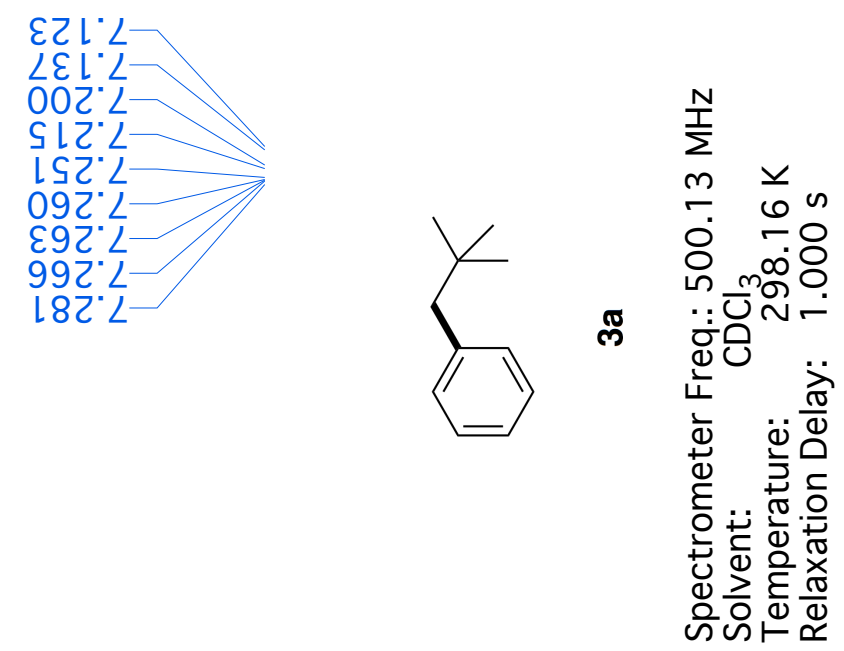


$\angle \varepsilon S^{\circ} 62$

888' $L$

90t:0s

906.9L

E $9 L^{\circ} L L$

Sカ8.9己L—

$\forall \varepsilon L^{\circ} \angle Z L$

0Z9"0ह L

८88" 6 \& L

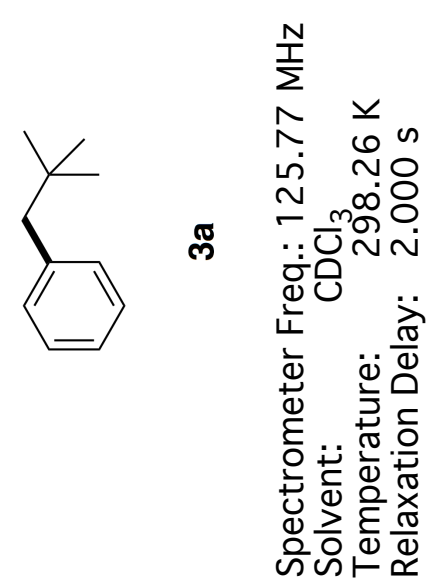


LE6. L

$S \angle 6^{\circ} \mathrm{L}$

$\rightarrow 66^{\circ} \mathrm{L}$

$\checkmark 10{ }^{\circ} 2$

$9 \triangleright 9^{\circ} 2$

S99.

$\rightarrow 89^{\circ} 2$
$06 L^{2} L$

902"

$2 \angle 2 \cdot \angle$

$182^{\circ} \mathrm{L}$

$162{ }^{\circ} L$

$0 I E^{\circ} L$
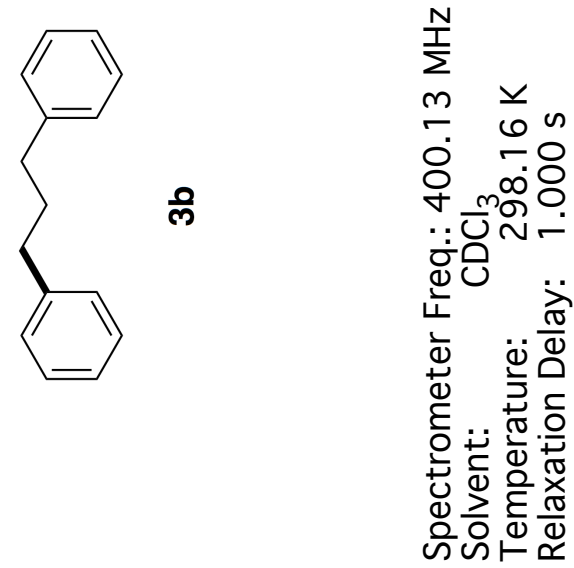
$\varepsilon 60^{\circ} \varepsilon \varepsilon$

¿ $8 \varsigma^{\circ} \varsigma \mathcal{E}$

906:9L

Elt: $L L$

998. 92

乙E†'8ट L

L $\angle S " 8 Z$ L

てレナでレ

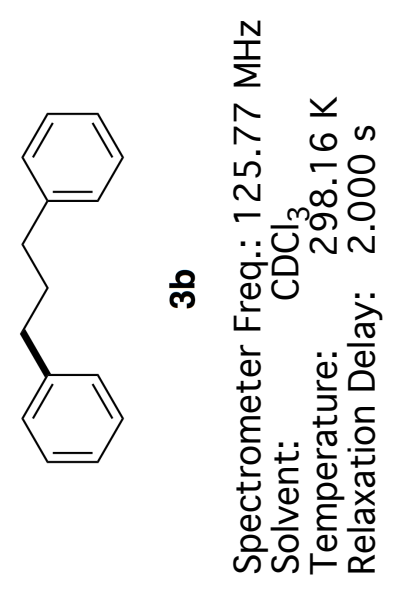



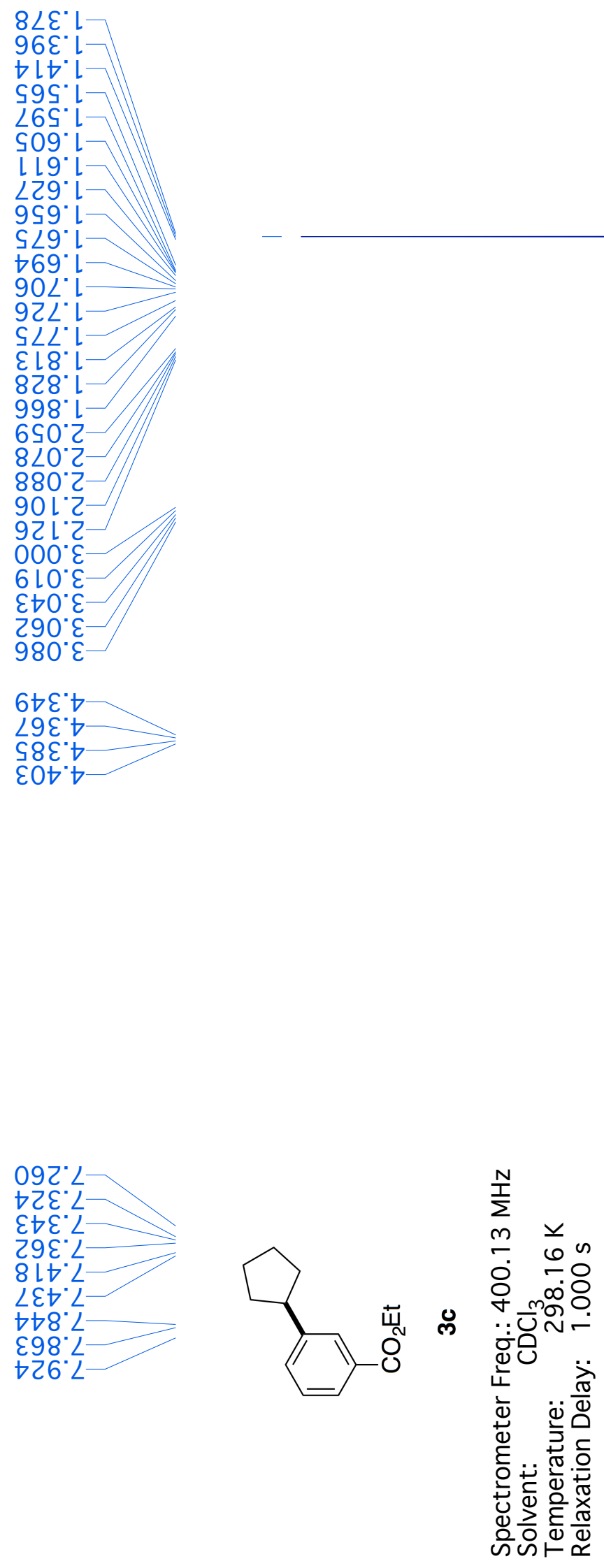
SES"ヤ

\& $\angle 9^{\circ} \varsigma 乙$

$\varepsilon S\llcorner " \nabla \varepsilon$

0ฤ6.ง

910 19

LS6.9

$O L C^{\circ} L L$
$\rightarrow 9 \nabla^{\circ} L L$

$8 \mathrm{~L} \mathrm{~L}^{\circ} \angle \mathrm{Z}$

ع9ع.82

069.62 L

8LS'OEL

$\neg \succ 8^{\circ} L \mathcal{E}$ L

6S6"9tl

$\angle S O^{\circ} \angle 9 L$

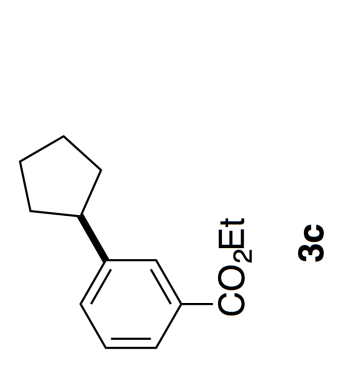

$\stackrel{N}{\Sigma}$

ヘ

in

mo

:ত্N

पे ते

㐫

凹 동

은 푼헝음

너을

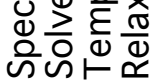

S58

유 
$\operatorname{lt9} 9^{\circ}$

$299^{\circ}$

ᄂS\& $8^{\circ}$

Z†9:

$169^{\circ} 2$

$888^{\circ} \mathrm{C}$

$\angle \mathcal{E} \mathcal{E}^{\circ}$

6S L'S

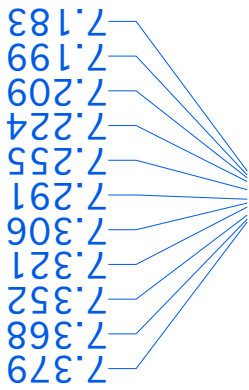

옹

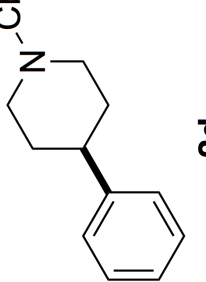

$\stackrel{N}{\Sigma}$

m $\underline{v}$

ர்

:บัN

ह్ల

亡ั. .. ते

๑̀

동

인

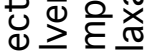

응잉 S59 
$662^{\circ} \varepsilon \varepsilon$

$8 S \angle 2 t$

$6 \angle L ' t D$

$\forall 12^{\circ} \angle 9$

906.9L

O9L'LL

†SS.92

788.92

$\varepsilon \varepsilon 0.82$
SOl.82

$0 \varepsilon 9.82$

$9 \angle 9^{\circ} 82$

ISO" $\angle E L$ L

089'St L

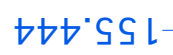

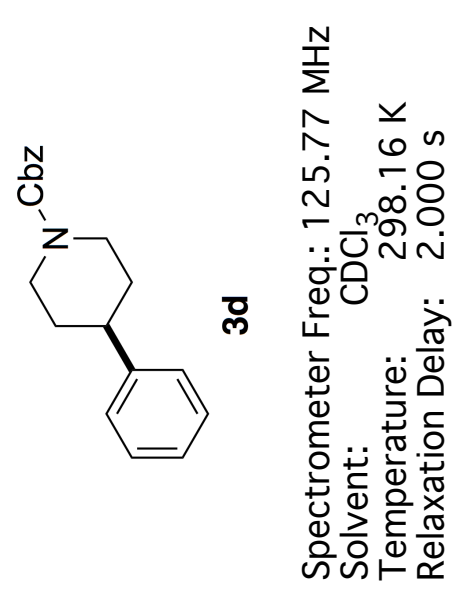




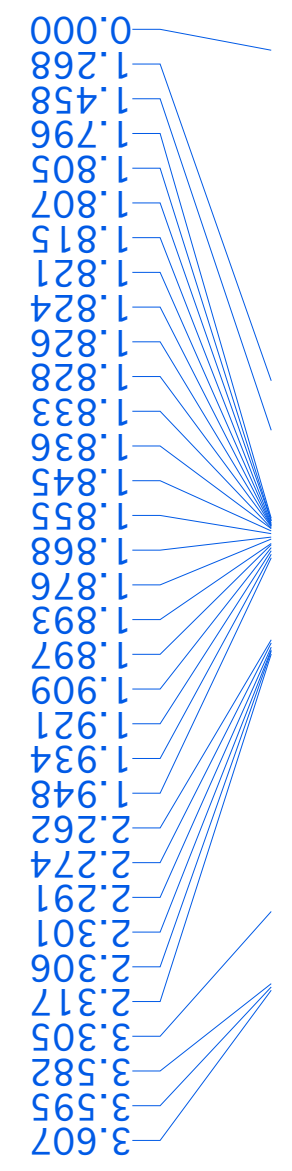

\section{LL8'}

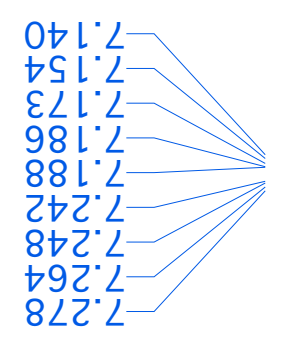


OLE' $\varepsilon Z$

LSZ'8Z

$9+1 \cdot 9 \varepsilon$

$80 Z^{\prime} \angle t$

sst. 19

$\angle 06: 92$
$09 l^{\circ} \angle L$

Slt: $\angle 2$

$8 S 2 \cdot 6 L$

દ29.

$812.82 \mathrm{~L}$

L82 St L

$\downarrow 69^{\circ} \mathrm{tSL}$

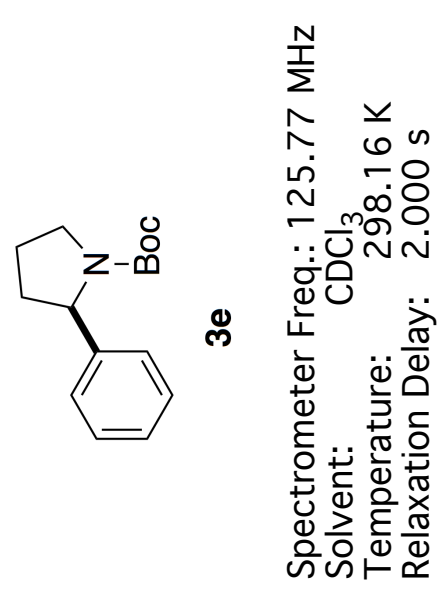



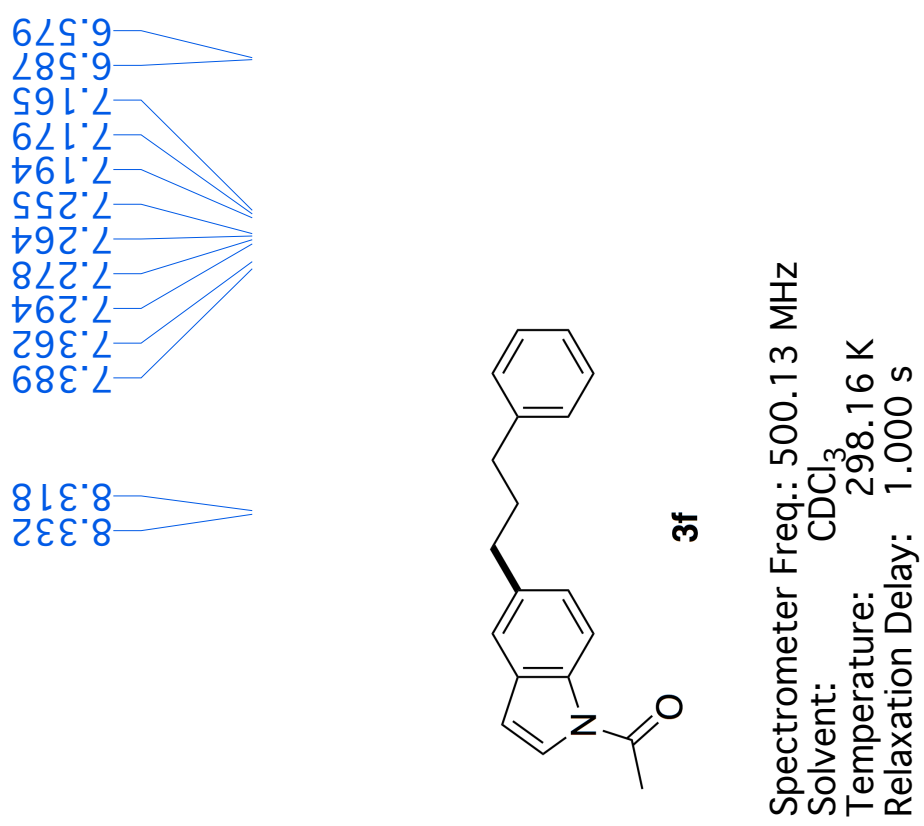
L86'ย乙-

$\neg 6 \nabla^{\circ} \varepsilon \varepsilon$

69t" $\varepsilon$

LZS'SE

S06.9L

$09 L^{\circ} L L$

$\varepsilon L t^{\prime} L L$

LSL"60

6SE.0Z

†レ"Sट

$6 \varepsilon 8$.

6 เt.82

699.82

O8L"OE

9乙L"†ยL-

$\angle \angle 8^{\circ} \angle E L$

くヤザてヤに-

095.89 L

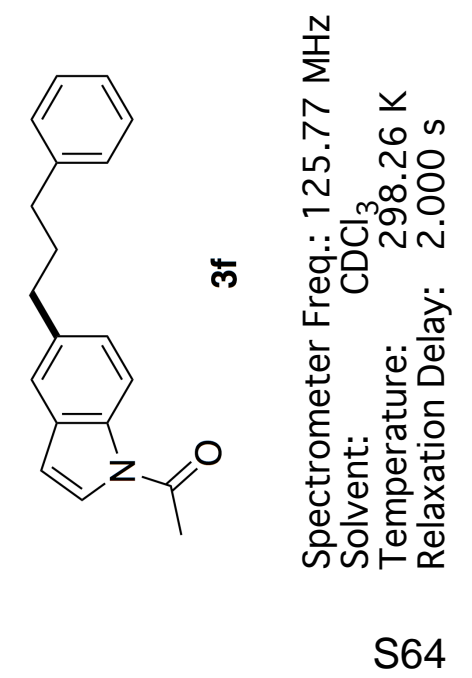




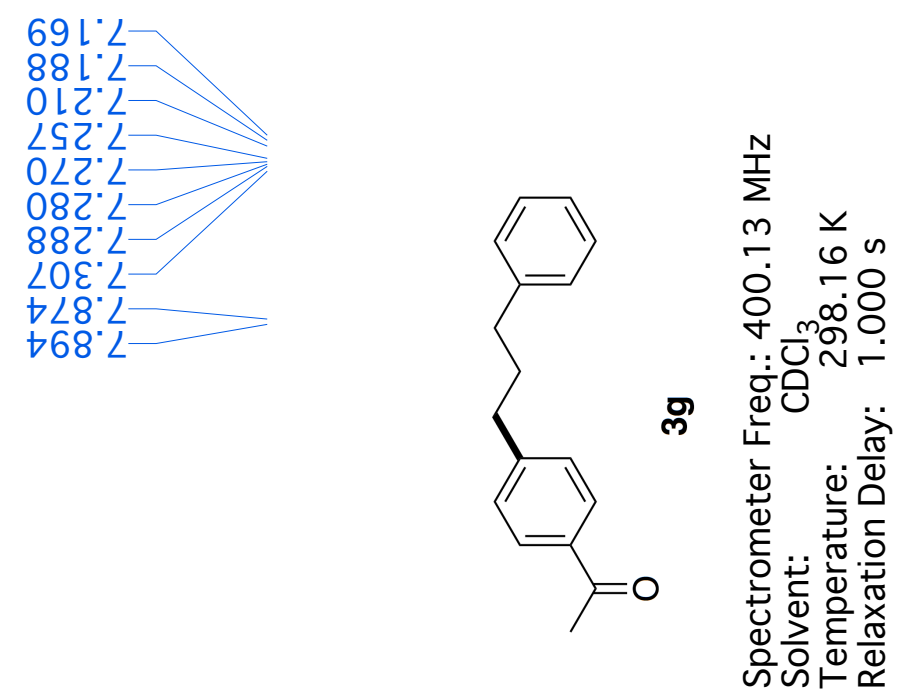


8†乙"8t L

LE $6^{\circ} \angle 6^{\circ}$

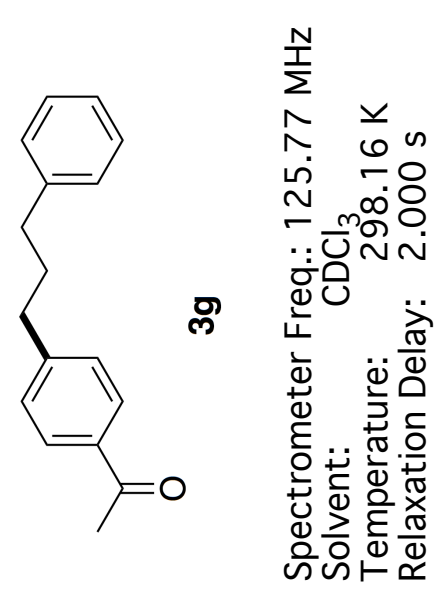


268

$\varepsilon 26$

6ह6.

$\rightarrow 56^{\circ}$

$\varepsilon \angle Z^{\circ} Z$

$829^{\circ}$

$\rightarrow \succ 9^{\circ}$

099.

$\rightarrow 69^{\circ} 2$

$60<2$

$\rightarrow Z L \cdot 2$
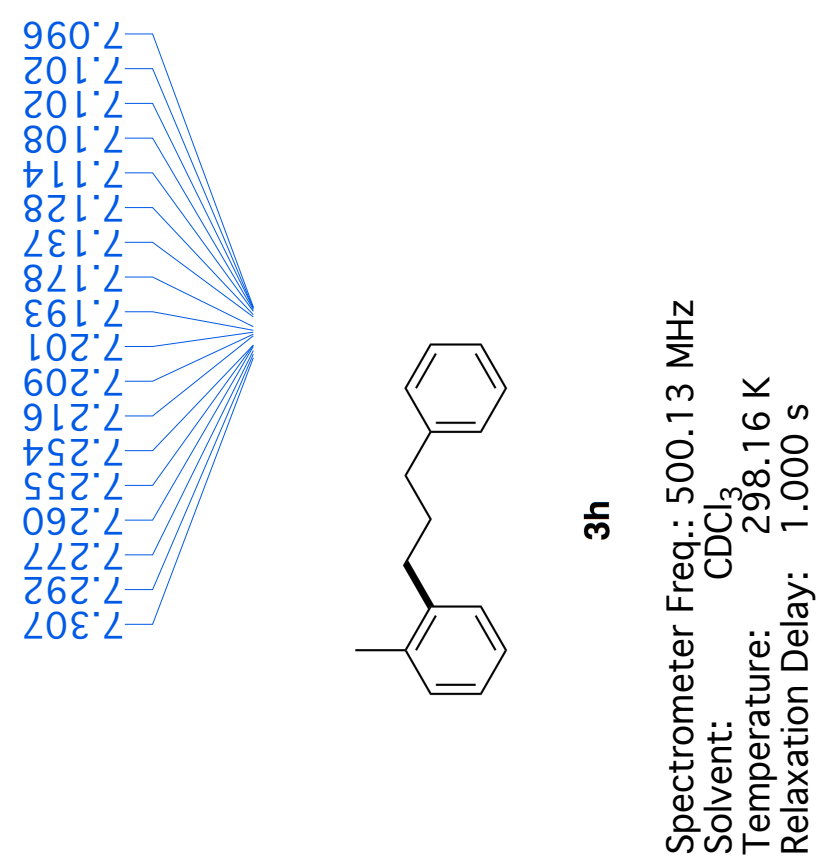

$9 \angle 8^{\circ}$ $\rightarrow 68^{\circ} \mathrm{L}$

E L $6^{\circ}$

LE6

OS6.

$8 \mathrm{Ll} 2$

$6 เ \nabla^{\circ} 2$

$\angle E \nabla^{\circ} 2$

SSt"

909.2

S29.

$\downarrow 七 9^{\circ} 2$
E9 L L

08 L

L L 2

092 "

$892^{\circ} \mathrm{L}$

982" 2

$\downarrow 0 \mathcal{E}^{\circ} \mathrm{L}$

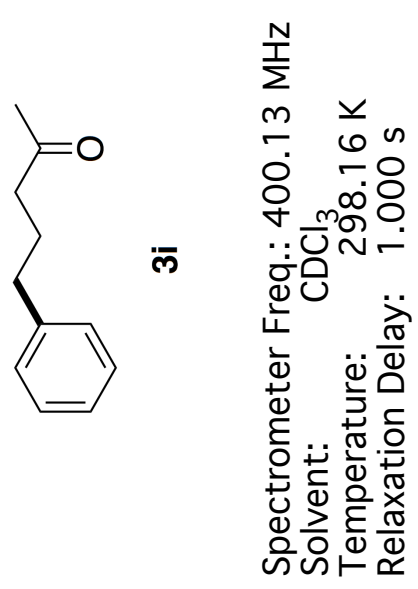

$\left.{ }^{-\infty}\right|_{-m} ^{\infty}$

궁

6

-

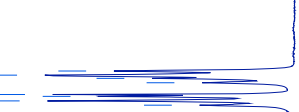

กัด ำก 
$\angle O \varepsilon^{\circ} S Z$

$0 \rightarrow 00^{\circ} 0 \varepsilon$

$82 L^{\circ} S \varepsilon$

$\angle 26^{\circ} 2 \downarrow$

S06.9L

Elt:LL

St0.92 L $\angle \angle t^{\circ} 82 L$

2SS.82 L

999・レい

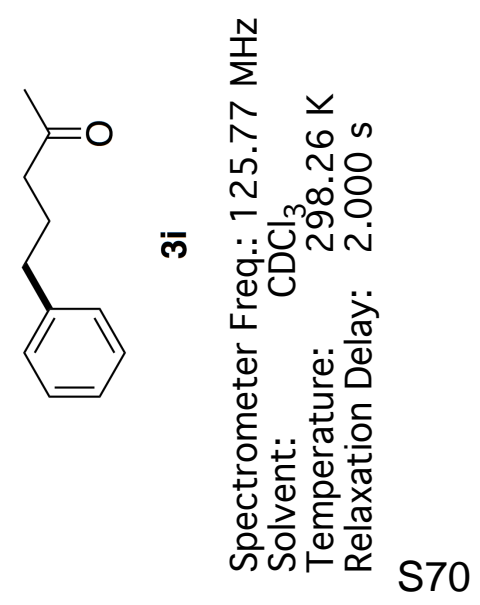




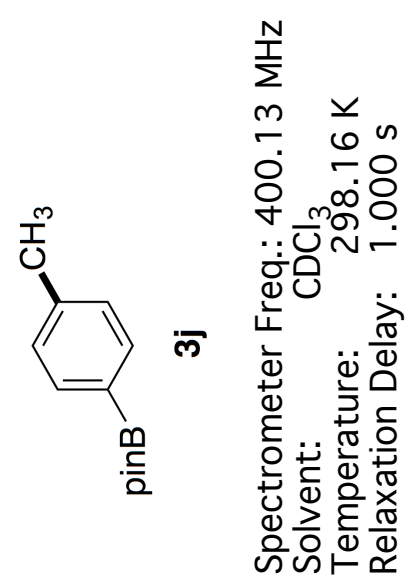




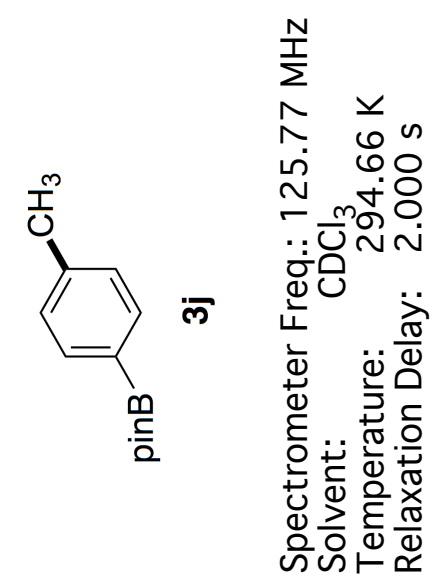




\section{†St"L \\ $\angle \angle D^{\circ} L$ \\ 958. \\ ZL8. \\ 688.}

L06" L

LE6.

$6 \nabla 6$

$\angle \angle 0^{\circ}$

tOL' $\mathrm{C}$

$\angle L L ' Z$

$9 \varepsilon{ }^{2} \cdot 2$

685.

E09.

† $29^{\circ} \mathrm{Z}$

LE9 2

L $599^{\circ} \mathrm{C}$

999"Z

$8 \angle 9^{\circ}$

$589^{\circ} \mathrm{C}$

ह69.

\section{ZLL'Z}

\section{$82 L$}

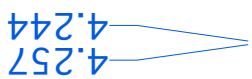

$\angle S Z^{\circ}$

$\varepsilon 90^{\circ} \mathrm{s}$

$180^{\circ} \mathrm{S}$

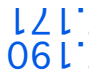

$06 L^{\circ} \cdot 2$

$092^{\circ} \mathrm{L}$ $582^{\circ} L$

$\rightarrow 0 \varepsilon^{\circ} L$

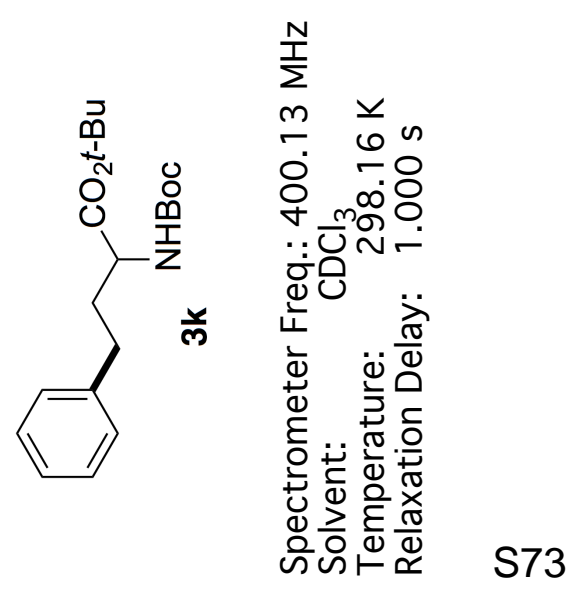


L L

งहt" 82

$9 L L L E$

$986^{\circ} \varepsilon S$

$\angle 06^{\prime} 9 L$

$09 L^{\circ} L$

$80 \angle{ }^{\circ} 6 L$

S56.18

9tL.92

OSt. 82

oOع゙เレ

LSt'SS L

$\angle 88^{\circ} L \angle L$

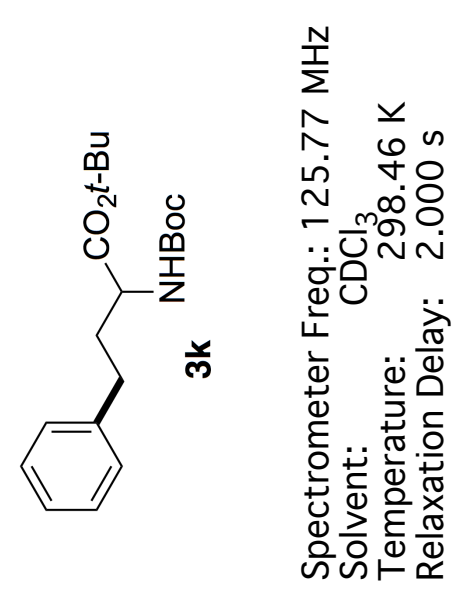


$96 \varepsilon^{\circ} \mathrm{L}$

$\varepsilon \succ 0^{\circ} \varepsilon$

$\angle S O^{\circ} \mathcal{-}$

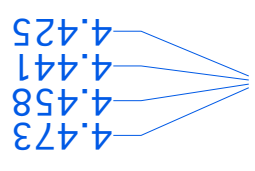

$\angle \angle 6^{\circ} D$

$\rightarrow 66^{\circ} \rightarrow$
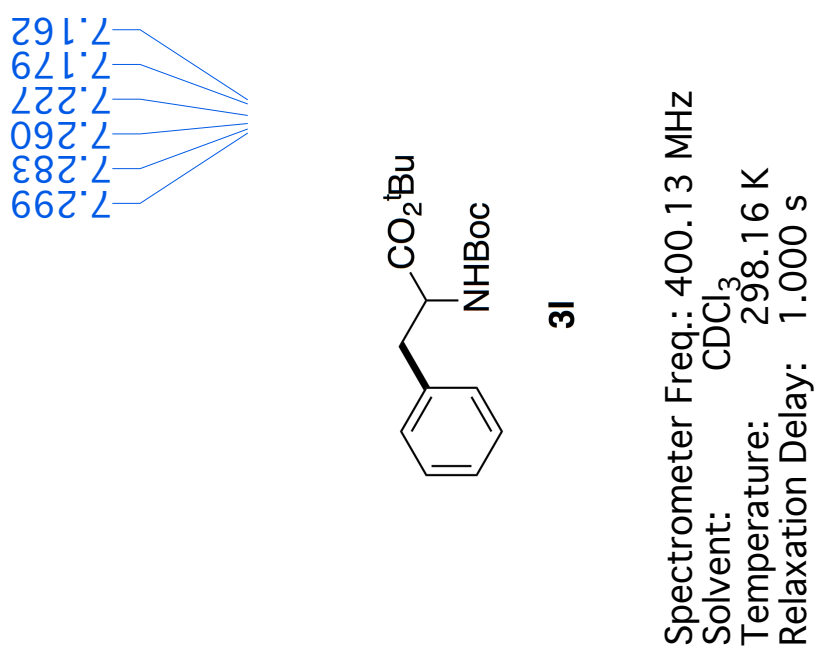

$\underset{+\infty}{\infty}$

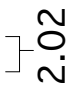

$=\left\{\begin{array}{l}\bar{\infty} \\ =\infty\end{array}\right.$

$-6$

$\frac{\varepsilon}{2}$ 
$2500^{\circ} 82$

$9 \varepsilon b^{\circ} 82$

$099^{\circ} 8 \varepsilon$

$9 s 6^{\circ} t s$

S06.9L

$091^{\circ} L$

$\varepsilon L t: L L$

$8 L 0^{\circ} 62$
806.92 L-

St9.62L

LES" $9 \varepsilon \mathrm{L}$

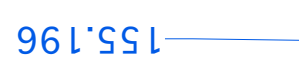

$990^{\circ} \mathrm{L} \angle L$

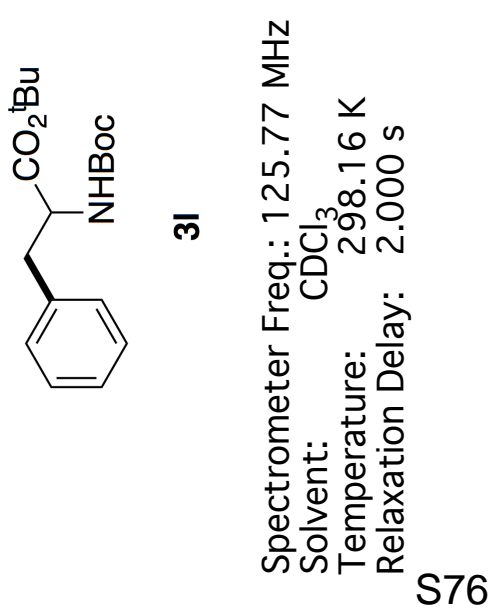




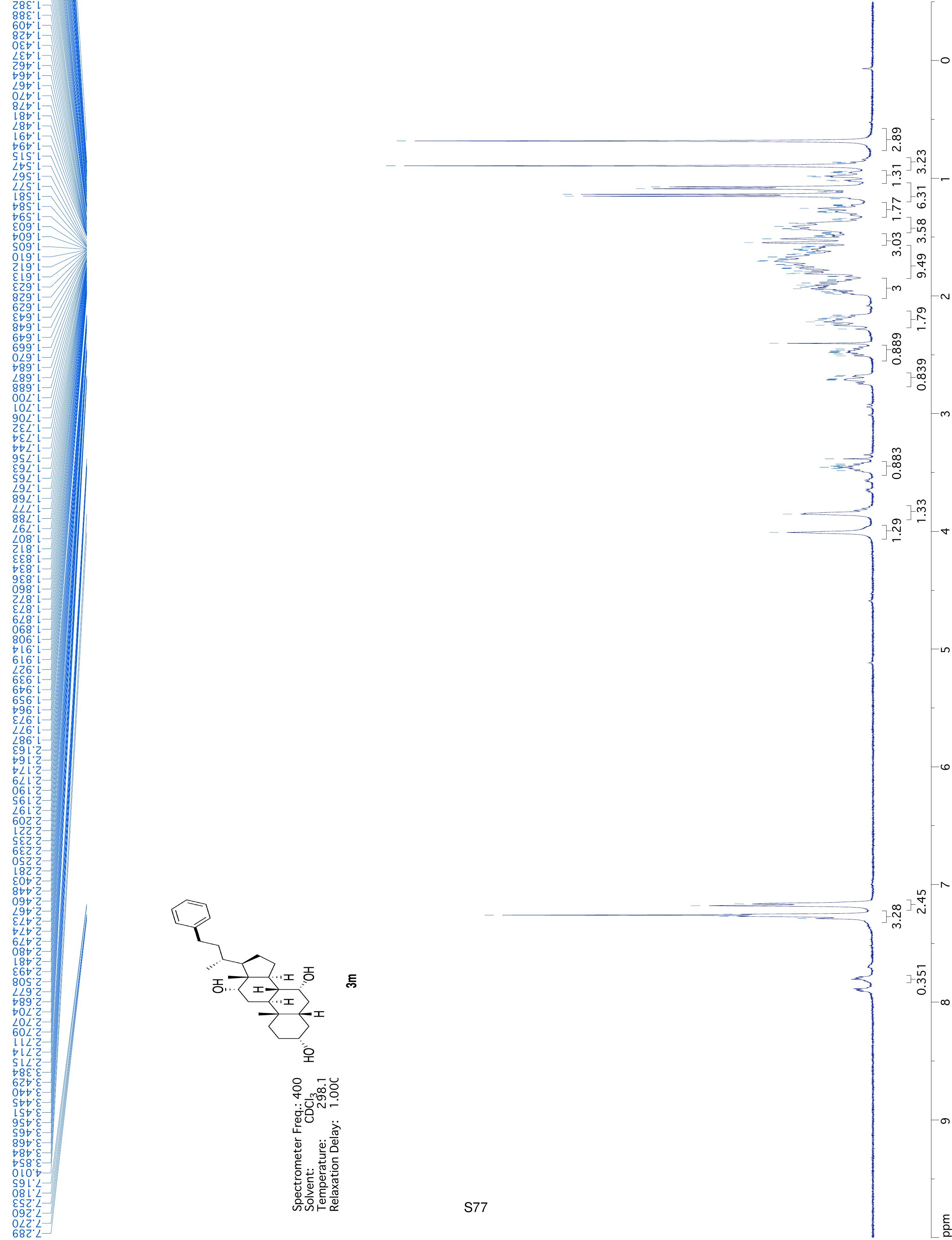


289.2

268. L L

899.22

9SE' $\varepsilon ट$

629.हट

SLL"9Z

$\angle 89^{\circ} \angle 2$

\& $6 \varepsilon^{\circ} 82$

$9 \angle 9^{\circ} 0 \varepsilon$

$\varepsilon 8 \angle$ ' $2 \varepsilon$

9SL" $\nabla \varepsilon$

$9 \angle 8 " \nabla \varepsilon$

$06 \varepsilon^{\prime} \varsigma \varepsilon$

हट9"

L L L $8 \varepsilon$

$\angle \varepsilon \angle{ }^{\circ} 6 \varepsilon$

$\rightarrow 08.6 \varepsilon$

Z29. Lt

100 ' 27

$96 \varepsilon^{\circ} \mathrm{t} \succ$

0ร9.9t

ZZt $\angle t$

009:89

L L L 'ZL

$0 \varepsilon \mathcal{C}^{\prime} \varepsilon L$

O9L LL

ヤレ゙ $L L$

てヤじヤてい—

टS9. SटL

20t"82 L

†6॰ $82 \mathrm{~L}$

$\varepsilon\left\llcorner 6^{\prime} \triangleright \varepsilon\llcorner\right.$

$\varepsilon \varepsilon \nabla^{*} \varepsilon \triangleright$<smiles>CCc1ccccc1</smiles>

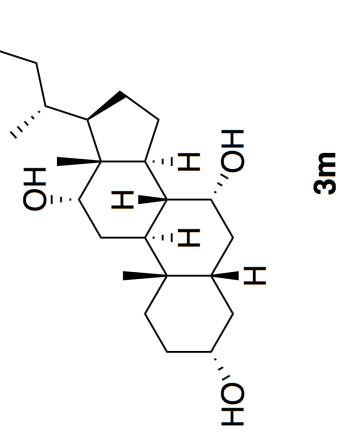

$\stackrel{N}{N}$

$\curvearrowright \quad \frac{v}{0}$

ஸ்

- mুN

㐫 穴

니

离

등 푼 은

넌 드릉

ํํㅇ $\frac{E}{0} \frac{\pi}{0}$

फuा

S78 
$822^{\circ} L$
$972{ }^{\circ}$
$\rightarrow 92^{\circ}$

\&68.

L L6.

0\&6.

8†6"

$\angle 96^{\circ}$

$\Delta t t^{\circ}$

$29 t^{\circ} 2$

08t

$\angle \supset S^{\circ} 2$

E9

$6 \angle S^{\circ}$

$709^{\circ}$

E29.

L $59^{\circ} \mathrm{Z}$

$\angle 99^{\circ} \mathrm{Z}$

E89. $\mathrm{Z}$

$669^{\circ} 2$
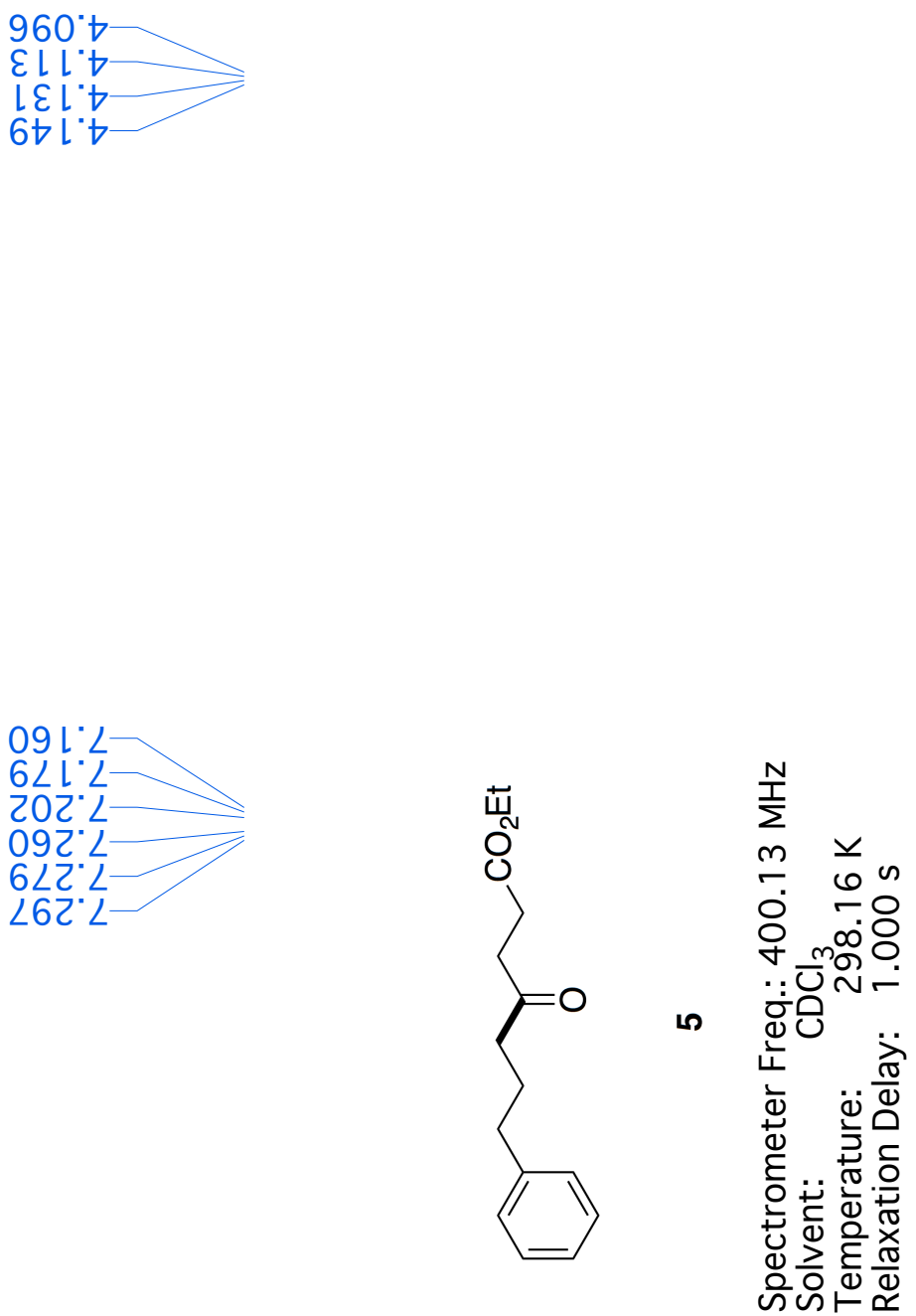

$\exists \stackrel{\infty}{\sim}$
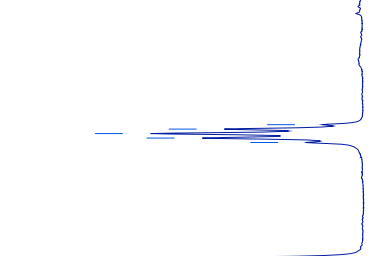

$\checkmark N$

-

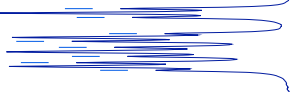

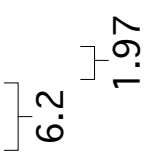

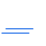

?
$-\pi$

$-6$

$\infty$ 
†ย乙๋ヤ

$092: 92$

$\angle 60^{\circ} 9 \varepsilon$

$606^{\circ} \mathrm{lt}$

૬ع9"09

$206.9 L$
$091.2 L$

$S I t^{\circ} L L$

166. 921

$\angle Z S^{\circ} 821$

819.tt

$028^{\circ} 2 \angle L$

SLL'80Z

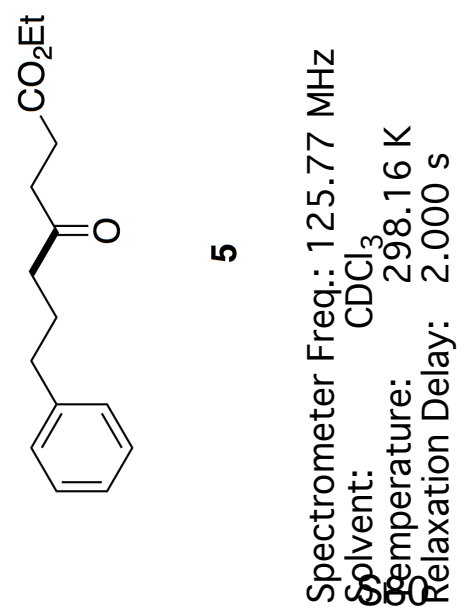

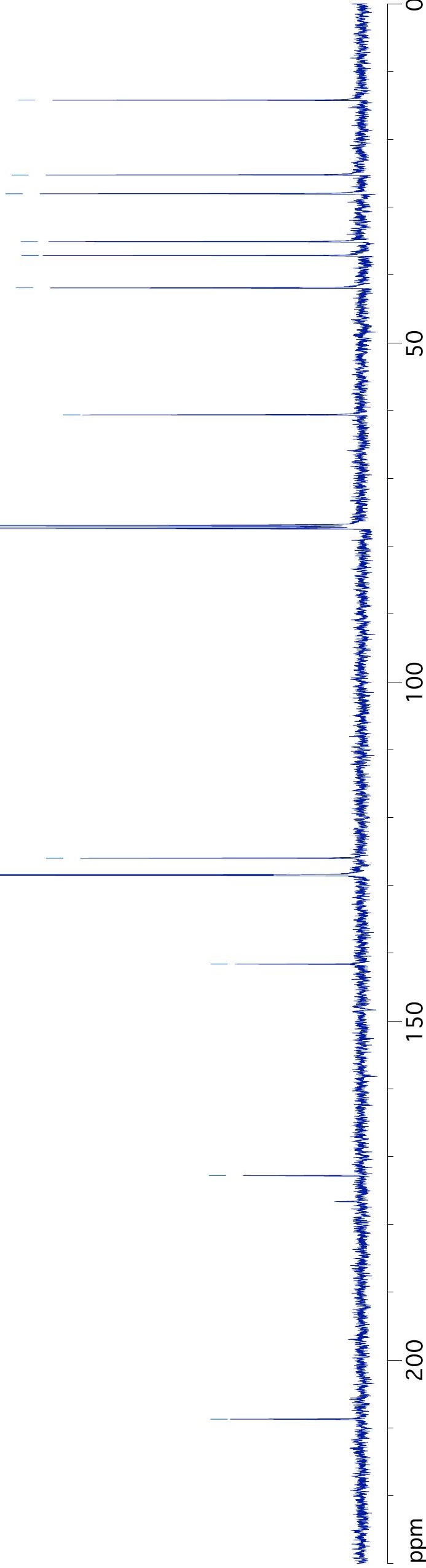


$L \angle E^{*} L-$
$62 D^{\circ} L-$

$9+0.2$

$856^{\circ} 2$
$\underline{E}$

$\sqrt{2}$

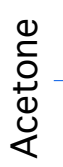

$\infty$

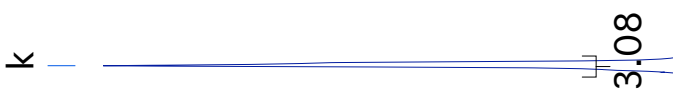

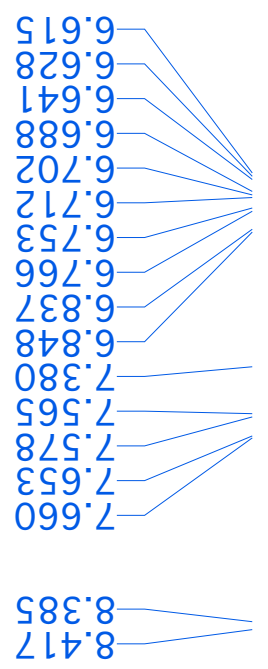

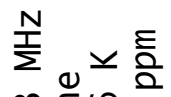

m

+

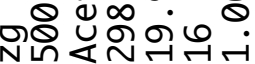

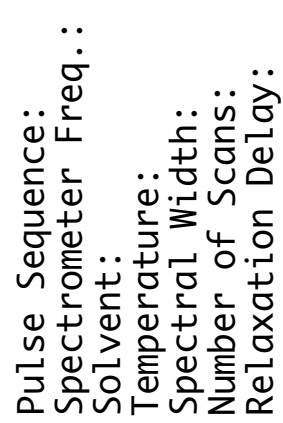

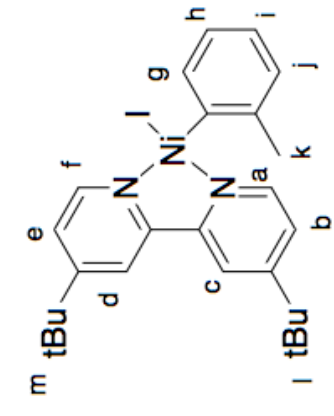

S81

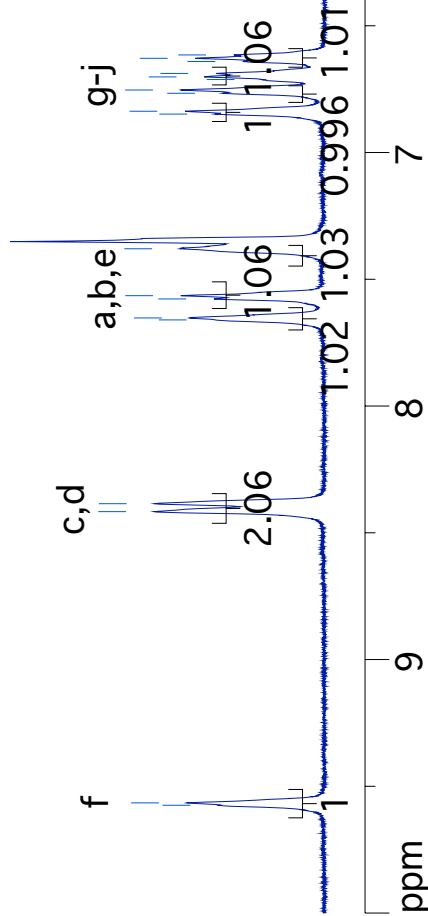




\section{$9 \angle L \cdot 9 \varepsilon$}

$9 \varepsilon 9^{\circ} t s$

Stl.69

LSZ"6L L

$919^{\circ} 22$

$6 \angle 2 \div 2$

$\varepsilon \angle \varepsilon^{\circ} \nabla 2 L$

$96 L " \angle Z L$

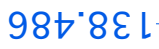

टحち・E†

S96.8t L

$618.6 t$

SEO" $\neg S L$

96 L' $\nabla S L$

Lt9"9s L

2LO००9L

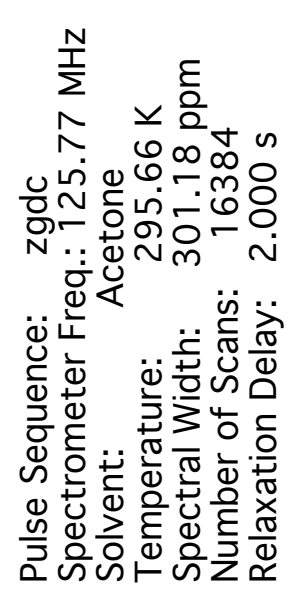

ธเナ゙ฑ9L 